ISSN 1855-3966 (printed edn.), ISSN 1855-3974 (electronic edn.)

ARS MATHEMATICA CONTEMPORANEA 17 (2019) 653-698

https://doi.org/10.26493/1855-3974.1813.7ae

(Also available at http://amc-journal.eu)

\title{
Reconfiguring vertex colourings of 2-trees
}

\author{
Michael Cavers, Karen Seyffarth \\ Department of Mathematics and Statistics, University of Calgary, \\ Calgary, AB T2N 1 N4 Canada
}

Received 4 October 2018, accepted 8 October 2019, published online 16 December 2019

\begin{abstract}
Let $H$ be a graph and let $k \geq \chi(H)$ be an integer. The $k$-colouring graph of $H$, denoted $G_{k}(H)$, is the graph whose vertex set consists of all proper $k$-vertex-colourings (or simply $k$-colourings) of $H$ using colours $\{1,2, \ldots, k\}$; two vertices of $G_{k}(H)$ are adjacent if and only if the corresponding $k$-colourings differ in colour on exactly one vertex of $H$. If $G_{k}(H)$ has a Hamilton cycle, then $H$ is said to have a Gray code of $k$-colourings, and the Gray code number of $H$ is the least integer $k_{0}(H)$ such that $G_{k}(H)$ has a Gray code of $k$-colourings for all $k \geq k_{0}(H)$. Choo and MacGillivray determine the Gray code numbers of trees. We extend this result to 2-trees. A 2-tree is constructed recursively by starting with a complete graph on three vertices and connecting each new vertex to an existing clique on two vertices. We prove that if $H$ is a 2-tree, then $k_{0}(H)=4$ unless $H$ is isomorphic to the join of a tree $T$ and a vertex $u$, where $T$ is a star on at least three vertices, or the bipartition of $T$ has two even parts; in these cases, $k_{0}(H)=5$.
\end{abstract}

Keywords: 2-trees, graph colouring, Gray codes, Hamilton cycles, reconfiguration problems.

Math. Subj. Class.: 05C15, 05C45

\section{Introduction}

Let $H$ be a graph and $k$ a positive integer. We define a proper $k$-vertex-colouring of $H$ as a function $f: V(H) \rightarrow\{1,2, \ldots, k\}$ for which $f(x) \neq f(y)$ for any $x y \in E(H)$. Since we are concerned only with proper $k$-vertex-colourings, we use the simpler term $k$-colouring, and refer to $f(x)$ as the colour of $x$. For notation and terminology not defined here, the reader is referred to Bondy and Murty [1].

A graph $H$ has a Gray code of $k$-colourings if it is possible to list all the $k$-colourings of $H$ in such a way that consecutive colourings in the list (including the last and the first) differ on exactly one vertex of $H$, and the Gray code number of $H$ is the least integer

E-mail addresses: mcavers@ucalgary.ca (Michael Cavers), kseyffar@ucalgary.ca (Karen Seyffarth) 
$k_{0}(H)$ for which $H$ has a Gray code of its $k$-colourings for all $k \geq k_{0}(H)$. Equivalently, we may define the $k$-colouring graph of $H$, denoted $G_{k}(H)$, to be the graph whose vertices correspond to all $k$-colourings of $H$, and whose edges connect two $k$-colourings of $H$ that differ on exactly one vertex of $H$. In this context, $H$ has a Gray code of $k$-colourings if and only if $G_{k}(H)$ has a Hamilton cycle, and the Gray code number of $H$ is the the least integer $k_{0}(H)$ for which $G_{k}(H)$ has a Hamilton cycle for all $k \geq k_{0}(H)$.

The $k$-colouring graph is an example of a reconfiguration graph. Such graphs are often used in the study of what are known as reconfiguration problems. Generally, a reconfiguration problem asks: given two (different) feasible solutions to a problem, can one solution be transformed to the other through a sequence of allowable moves, while maintaining feasibility at each stage? In the context of $k$-colourings, the $k$-colouring graph is connected if and only if any $k$-colouring can be reconfigured into any other $k$-colouring by re-colouring one vertex at a time in such a way that each intermediate colouring is a $k$-colouring. Recently, reconfiguration problems have been receiving wide attention, and have been studied for various graph colouring problems [2, 3, 4, 10, 15], for dominating sets [12, 13, 18], and for various other graph problems including vertex covers, cliques, and independent sets [14].

The $k$-colouring graph arises in the context of theoretical physics, where it is the graph of the Glauber dynamics Markov chain; the goal is to find efficient algorithms for almost uniform sampling of $k$-colourings of graphs [16]. The Glauber dynamics Markov chain converges to the uniform distribution provided that the $k$-colouring graph is connected, prompting Cereceda, van den Heuvel and Johnson [4] to ask the question: given a graph $H$ and a positive integer $k$, is $G_{k}(H)$ connected? They prove that if $H$ has chromatic number $k \in\{2,3\}$, then $G_{k}(H)$ is never connected, whereas for $k \geq 4$, there are $k$ chromatic graphs $H$ for which $G_{k}(H)$ is connected, and other $k$-chromatic graphs $H$ for which $G_{k}(H)$ is not connected. In general, they prove that $G_{k}(H)$ is connected for all $k \geq \operatorname{col}(H)+1$, where $\operatorname{col}(H)$, the colouring number of $H$, is defined as $\operatorname{col}(H):=$ $\max \{\delta(G) \mid G \subseteq H\}+1$. A slightly weaker version of this result is proven in [8].

Choo and MacGillivray [6] initiated the study of Hamilton cycles in $k$-colouring graphs by proving that the Gray code number of $H$ is well defined, i.e., $G_{k}(H)$ has a Hamilton cycle for all $k \geq \operatorname{col}(H)+2$. This gives the upper bound $k_{0}(H) \leq \operatorname{col}(H)+2$. Note that if $T$ is a tree, then $\operatorname{col}(T)=2$ and $G_{2}(T)$ is disconnected; hence $3 \leq k_{0}(T) \leq 4$. Choo and MacGillivray [6] determine which trees have $k_{0}(T)=3$ and which have $k_{0}(T)=4$. In particular, they prove that if $T$ is a tree, then $k_{0}(T)=3$ unless $T \cong K_{1,2 \ell}$, for $\ell \geq 1$, in which case $k_{0}(T)=4$. They also determine the Gray code numbers of complete graphs and cycles. Celaya, Choo, MacGillivray and Seyffarth [3] establish the Gray code numbers of complete bipartite graphs. Haas [11] studies a variation of $k$-colouring graphs, namely, canonical colouring graphs, and uses techniques developed in [6] to show that canonical colouring graphs of trees have Hamilton cycles.

Given the results for trees and complete graphs, a natural question is to determine the Gray code numbers of $k$-trees. We use the definition of $k$-tree given in [9], that is, a $k$-tree is constructed recursively by starting with a complete graph on $k+1$ vertices and connecting each new vertex to an existing clique on $k$ vertices (hence a 1-tree is simply a connected acyclic graph). A vertex of degree $k$ in a $k$-tree is called a leaf. Let $H$ be a $k$-tree. Then it is clear that the chromatic number of $H$ is $\chi(H)=k+1$, and that $G_{k+1}(H)$ is disconnected. Since every induced subgraph of $H$ has a leaf, $\operatorname{col}(H)=k+1$. Thus it follows from [6, Theorem 3.4] that $k_{0}(H) \leq k+3$, and therefore $k+2 \leq k_{0}(H) \leq k+3$. The problem 
is therefore reduced to classifying $k$-trees into those with Gray code number $k+2$ and those with Gray code number $k+3$. The answer appears to be far from trivial. In the current paper, we provide a complete solution for 2-trees; the characterization can be stated in fairly non-technical language, but the proof involves numerous cases and generalizations of the techniques used in [3,6]. The join of graphs $G_{1}$ and $G_{2}$, denoted by $G_{1} \vee G_{2}$, is obtained from the disjoint union of $G_{1}$ and $G_{2}$ by adding all edges between vertices of $G_{1}$ and vertices of $G_{2}$.

Theorem 1.1. If $H$ is a 2-tree then $k_{0}(H)=4$, unless $H \cong T \vee\{u\}$ for some tree $T$ and vertex $u$, where $T$ is a star on at least three vertices or the bipartition of $V(T)$ has two even parts; in these cases, $k_{0}(H)=5$.

The remainder of the paper is devoted to proving this theorem. We first characterize 2 -trees of diameter two (Lemma 3.2). We then determine the 2 -trees, $H$, of diameter two for which $G_{4}(H)$ has a Hamilton cycle (Lemmas 3.3 and 3.5). This is done by considering the structure of $G_{3}(T)$, where $T$ is a tree (Lemmas A.2 and 3.6). We show that if $H$ is a 2-tree with diameter at least three, then $G_{4}(H)$ has a Hamilton cycle (Lemmas 6.3 and 6.4). To do so we describe a specific recursive procedure for constructing 2-trees of diameter at least three (Theorem 4.3).

\section{Preliminaries}

Definition 2.1. Let $H$ be a graph, and let $X$ and $Y$ be disjoint subsets of $V(H)$. We denote by $[X, Y]$ the set of edges of $H$ that have one end in $X$ and the other end in $Y$.

Definition 2.2. Let $H$ be a graph and $L$ a function that assigns to each vertex $v \in V(H)$ a set of positive integers, $L(v)$, called the list of $v$. An $L$-colouring of $H$ (also called a list colouring of $H$ with respect to $L$ ) is a (proper) colouring $c: V(H) \rightarrow \mathbb{N}$ such that $c(v) \in L(v)$ for each $v \in V(H)$. We define the $L$-colouring graph of $H$, denoted $G_{L}(H)$, to be the graph whose vertex set consists of all $L$-colourings of $H$; two $L$-colourings are joined by an edge of $G_{L}(H)$ if they differ in colour on just one vertex of $H$.

Remark 2.3. If $k \geq \chi(H)$ and $L(v):=\{1,2, \ldots, k\}$ for each $v \in V(H)$, then $G_{L}(H) \cong$ $G_{k}(H)$, the $k$-colouring graph of $H$.

We use $G \square H$ to denote the Cartesian product of graphs $G$ and $H$, and $Q_{n}$ to denote the $n$-dimensional hypercube, defined as the Cartesian product of $n$ copies of $K_{2}$.

Remark 2.4. Let $H_{1}$ and $H_{2}$ be vertex disjoint graphs and let $L$ be an assignment of lists to the vertices of $\mathrm{H}_{1} \cup \mathrm{H}_{2}$. Then

$$
G_{L}\left(H_{1} \cup H_{2}\right)=G_{L}\left(H_{1}\right) \square G_{L}\left(H_{2}\right) .
$$

Lemma 2.5. Let $H$ be a 2 -tree with clique $X=\left\{x_{1}, x_{2}, \ldots, x_{\ell}\right\}$ where $\ell \leq 3$, and let $L$ be an assignment of lists to the vertices of $H$ so that
1. $\left|L\left(x_{i}\right)\right|=1$ and $L\left(x_{i}\right) \subseteq\{1,2,3,4\}, 1 \leq i \leq \ell$;
2. $L\left(x_{i}\right) \neq L\left(x_{j}\right)$ for all $1 \leq i \neq j \leq \ell$;
3. $L(v)=\{1,2,3,4\}$ for each $v \in V(H) \backslash X$. 
Then $G_{L}(H)$ has a spanning tree $T$ with $\Delta(T) \leq 4$.

Proof. The proof is by induction on $|V(H)|$. For the basis, $H \cong K_{3}$ with $V(H):=$ $\left\{v_{1}, v_{2}, v_{3}\right\}$. When $\ell=0, G_{L}(H)=G_{4}\left(K_{3}\right)$, which has a Hamilton cycle [6, Theorem 4.1], so take $T$ to be a Hamilton path in $G_{4}\left(K_{3}\right)$. When $\ell=1$, we may assume, without loss of generality, that $L\left(v_{1}\right):=\{1\}$ and $L\left(v_{2}\right)=L\left(v_{3}\right):=\{1,2,3,4\}$. Then $G_{L}(H) \cong G_{3}\left(K_{2}\right)$, which has a Hamilton cycle [6, Theorem 4.1], so take $T$ to be a Hamilton path in $G_{3}\left(K_{2}\right)$. When $\ell=2$, we may may assume, without loss of generality, that $L\left(v_{1}\right):=\{1\}, L\left(v_{2}\right):=\{2\}$, and $L\left(v_{3}\right):=\{1,2,3,4\}$. Then $G_{L}(H) \cong K_{2}$, and the result holds. Finally, when $\ell=3$, we may assume, without loss of generality, that $L\left(v_{1}\right):=\{1\}, L\left(v_{2}\right):=\{2\}$, and $L\left(v_{3}\right):=\{3\}$. Then $G_{L}(H) \cong K_{1}$, and the result holds.

Now suppose $H$ is a 2-tree with $|V(H)|>3$ and clique $X=\left\{x_{1}, \ldots, x_{\ell}\right\}$. Since $\ell \leq 3$, there is a leaf $v \in V(H)$ with $v \notin X$, and thus $H-v$ is a 2-tree containing the clique $X$. It follows by induction that $G_{L}(H-v)$ has a spanning tree $\mathcal{T}$ with $\Delta(\mathcal{T}) \leq$ 4. Let $V\left(G_{L}(H-v)\right):=\left\{f_{0}, f_{1}, \ldots, f_{N-1}\right\}$, and for each $f_{j} \in V\left(G_{L}(H-v)\right)$, let $F_{j} \subseteq V\left(G_{L}(H)\right)$ be the set of $L$-colourings of $H$ that agree with $f_{j}$ on $V(H-v)$. Then $\left\{F_{0}, F_{1}, \ldots, F_{N-1}\right\}$ is a partition of the vertices of $G_{L}(H)$. Since $v$ is a leaf of $H$, there are two ways to extend an $L$-colouring of $H-v$ to an $L$-colouring of $H$, and hence for each $j, 0 \leq j \leq N-1, F_{j}=\left\{a_{j}, b_{j}\right\}$ is a clique.

For each edge $f_{i} f_{j} \in E(\mathcal{T})$, there is a unique vertex $w \in V(H-v)$ for which $f_{i}(w) \neq$ $f_{j}(w)$. If $w v \notin E(H)$, then $\left[F_{i}, F_{j}\right]$ consists of two disjoint edges, and the subgraph of $G_{L}(H)$ induced by $F_{i} \cup F_{j}$ is a 4-cycle. Otherwise, $w v \in E(H)$, so $\left[F_{i}, F_{j}\right]$ has only one edge, and the subgraph of $G_{L}(H)$ induced by $F_{i} \cup F_{j}$ is a path of length three. In both cases, label the edge $f_{i} f_{j}$ in $\mathcal{T}$ with $\left|\left[F_{i}, F_{j}\right]\right|$.

Let $S$ denote the spanning subgraph of $G_{L}(H)$ corresponding to the spanning tree $\mathcal{T}$ of $G_{L}(H-v)$ as described above, that is, $S$ has edge set

$$
\left(\bigcup_{f_{i} f_{j} \in E(\mathcal{T})}\left[F_{i}, F_{j}\right]\right) \cup\left\{a_{j} b_{j} \mid 0 \leq j \leq N-1\right\} .
$$

Since $\left[F_{i}, F_{j}\right]$ is nonempty for each $f_{i} f_{j} \in E(\mathcal{T}), S$ is connected. Also, since $\Delta(\mathcal{T}) \leq 4$ and $\left[F_{i}, F_{j}\right]$ contains either one edge or two disjoint edges, $\Delta(S) \leq 5$. Furthermore, since there are only two vertices adjacent to $v$ in $H$, at most two edges incident to $f_{i}$ in $G_{L}(H-v)$ have label ' 1 '.

Let $S^{\prime}$ be the graph obtained from $S$ by deleting the edges $a_{j} b_{j}$ for each $f_{j} \in V(\mathcal{T})$ with $d_{\mathcal{T}}\left(f_{j}\right)=4$. Then $\Delta\left(S^{\prime}\right) \leq 4$, since if $a_{j} \in V(S)$ has $d_{S}\left(a_{j}\right)=5$, then $d_{\mathcal{T}}\left(f_{j}\right)=4$, and thus $d_{S^{\prime}}\left(a_{j}\right)=4$. We also claim that $S^{\prime}$ is connected. To prove this, it suffices to show that there is a path in $S^{\prime}$ from $a_{p}$ to $b_{p}$ for each $f_{p} \in V(\mathcal{T})$ with $d_{\mathcal{T}}\left(f_{p}\right)=4$. Suppose $f_{p} \in V(\mathcal{T})$ has $d_{\mathcal{T}}\left(f_{p}\right)=4$. Construct a path, $P$, in $\mathcal{T}$ starting at $f_{p}$, using edges labelled ' 2 ', and whose final vertex $f_{q}$ has $d_{\mathcal{T}}\left(f_{q}\right)<4$. Such a path exists since $\mathcal{T}$ has no cycles, and each degree four vertex in $\mathcal{T}$ is incident to at least two edges labelled ' 2 '. The union

$$
\left(\bigcup_{f_{i} f_{j} \in E(P)}\left[F_{i}, F_{j}\right]\right) \cup\left\{a_{q} b_{q}\right\}
$$

gives us a path in $S^{\prime}$ from $a_{p}$ to $b_{p}$. 
Therefore, $S^{\prime}$ is a connected spanning subgraph of $G_{L}(H)$, and thus $S^{\prime}$ has a spanning tree $T$ that is also a spanning tree of $G_{L}(H)$. Since $\Delta\left(S^{\prime}\right) \leq 4, \Delta(T) \leq 4$, thus completing the proof of the lemma.

The result in Lemma 2.5 is best possible in that $\Delta(T)$ cannot be reduced from four to three, as illustrated in the following example. Let $D$ denote the unique 2-tree of diameter three on six vertices, with vertices labelled as shown in Figure 1(a).

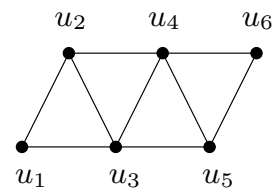

(a) $D$

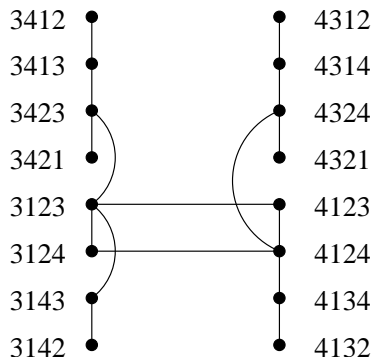

(b) $G_{L}(D)$

Figure 1: The 2-tree $D$ and $G_{L}(D)$.

Let $L: V(D) \rightarrow\{1,2,3,4\}$ be defined as follows.

$$
\begin{aligned}
L\left(u_{1}\right) & :=\{1\}, \\
L\left(u_{2}\right) & :=\{2\}, \text { and } \\
L\left(u_{i}\right) & :=\{1,2,3,4\} \text { for } 3 \leq i \leq 6 .
\end{aligned}
$$

If $f$ is an $L$-colouring of $D$, then $f\left(u_{1}\right)=1$ and $f\left(u_{2}\right)=2$, and thus we may denote the vertices of $G_{L}(D)$ by strings $i j k \ell$ where $f\left(u_{3}\right)=i, f\left(u_{4}\right)=j, f\left(u_{5}\right)=k$ and $f\left(u_{6}\right)=\ell$. Using this convention, $G_{L}(D)$ is depicted in Figure 1(b). Notice that $G_{L}(D)$ is unicyclic, and has exactly two nonadjacent vertices of degree four, ' 3123 ' and ' 4124 '. Thus every spanning tree of $G_{L}(D)$ has a vertex of degree four.

As part of their proof [6, Theorem 5.5], Choo and MacGillivray prove the following.

Remark 2.6. Let $G$ be a graph with vertex partition $\left\{F_{0}, F_{1}, \ldots, F_{N-1}\right\}$, such that

(i) $G\left[F_{i}\right]$ is a 4-cycle for each $i, 0 \leq i \leq N-1$;

(ii) $G\left[F_{i-1} \cup F_{i}\right]$ is isomorphic to either $P_{4} \square K_{2}$ or $Q_{3}$ for each $i, 1 \leq i \leq N-1$;

(iii) if $G\left[F_{i-1} \cup F_{i}\right]$ and $G\left[F_{i} \cup F_{i+1}\right]$ are both isomorphic to $P_{4} \square K_{2}$, then $G\left[F_{i-1} \cup\right.$ $\left.F_{i} \cup F_{i+1}\right]$ is not isomorphic to the graph in Figure 2.

Then $G$ has a Hamilton cycle.

The conditions imply that $\left[F_{i-1}, F_{i}\right] \neq \emptyset, 1 \leq i \leq N-1$, and hence there is a function, $h$, from a spanning subgraph of $G$ to a path $f_{0} f_{1} \cdots f_{N-1}$ of length $N-1$ defined by $h(u)=f_{i}$ for all $u \in F_{i}, 0 \leq i \leq N-1$.

In our next lemma, we adapt the result of Choo and MacGillivray to a more general scenario. Suppose $G$ is a graph with vertex partition $\left\{F_{0}, F_{1}, \ldots, F_{N-1}\right\}$ where $G\left[F_{i}\right]$ 


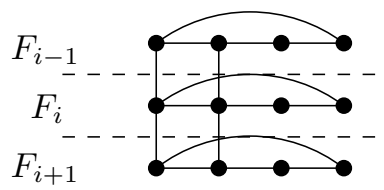

Figure 2: Forbidden subgraph.

contains a spanning cycle for each $i, 0 \leq i \leq N-1$ (these cycles form a 2 -factor of $G$ ). Further, assume that there is a function, $h$, from a spanning subgraph of $G$ to a tree with vertex set $\left\{f_{0}, f_{1}, \ldots, f_{n-1}\right\}$ such that $h(u)=f_{i}$ for all $u \in F_{i}, 0 \leq i \leq N-1$. The general idea is to choose, for each edge $f_{i} f_{j}$ of the spanning tree, appropriate edges from the set $\left[F_{i}, F_{j}\right]$ of $G$ so that we are able to construct a Hamilton cycle from among these edges and edges of the 2 -factor. See Figure 3 for an illustration of this result.

Lemma 2.7. Let $G$ be a graph with vertex partition $\left\{F_{0}, F_{1}, \ldots, F_{N-1}\right\}$, and let $T$ be a tree with $V(T):=\left\{f_{0}, f_{1}, \ldots, f_{N-1}\right\}$. Suppose there is a function, $h$, from a spanning subgraph of $G$ to $T$ such that $h(u)=f_{i}$ for all $u \in F_{i}, 0 \leq i \leq N-1$. Furthermore, suppose that for each $f_{i} f_{j} \in E(T), 0 \leq i, j \leq N-1$, there exist edges $e_{i, j}$ in $G\left[F_{i}\right]$ and $e_{j, i}$ in $G\left[F_{j}\right]$ such that

(i) if $j \neq k$ and $f_{i} f_{j}, f_{i} f_{k} \in E(T)$, then $e_{i, j} \neq e_{i, k}$;

(ii) if $e_{i, j}=a c$ and $e_{j, i}=b d$, then $G[\{a, b, c, d\}]$ contains a 4-cycle;

(iii) $G\left[F_{i}\right]$ has a Hamilton cycle $C_{i}$ such that

$$
M_{i}:=\left\{e_{i, j} \mid f_{i} f_{j} \in E(T)\right\} \subseteq E\left(C_{i}\right) .
$$

Then G has a Hamilton cycle $C$ such that

$$
\bigcup_{i=0}^{N-1}\left(E\left(C_{i}\right) \backslash M_{i}\right) \subseteq E(C)
$$

Proof. The proof is by induction on $N$. The result is trivial when $N=1$.

Let $N>1$. For each $f_{i} f_{j} \in E(T), 0 \leq i \leq N-1$, suppose $e_{i, j} \in E\left(G\left[F_{i}\right]\right), e_{j, i} \in$ $E\left(G\left[F_{j}\right]\right.$ ), and $C_{i}$ (a Hamilton cycle in $G\left[F_{i}\right]$ ) satisfy conditions (i), (ii) and (iii). Without loss of generality, we may assume that $f_{N-1}$ is a leaf of $T$, and that $f_{N-1} f_{N-2} \in E(T)$. Let $G^{\prime}:=G-F_{N-1}$ and $T^{\prime}:=T-f_{N-1}$. Using $e_{i, j}, e_{j, i}$ and $C_{i}$ as previously defined, $0 \leq i \leq N-2$, and $M_{i}$ as defined in (iii) except with $M_{N-2}$ replaced by $M_{N-2}^{\prime}:=$ $M_{N-2} \backslash\left\{e_{N-2, N-1}\right\}$, we apply the induction hypothesis to $G^{\prime}$. The result is a Hamilton cycle $C^{\prime}$ in $G^{\prime}$ such that

$$
\left(\bigcup_{i=0}^{N-3}\left(E\left(C_{i}\right) \backslash M_{i}\right)\right) \cup\left(E\left(C_{N-2}\right) \backslash M_{N-2}^{\prime}\right) \subseteq E\left(C^{\prime}\right),
$$

and $e_{N-2, N-1} \in E\left(C^{\prime}\right)$. Let $e_{N-2, N-1}:=a c, e_{N-1, N-2}:=b d$; without loss of generality, abdca is a 4 -cycle in $G[\{a, b, c, d\}]$, and hence

$$
C:=\left(C^{\prime}-e_{N-2, N-1}\right) \cup\left(C_{N-1}-e_{N-1, N-2}\right)+\{a b, c d\}
$$

is a Hamilton cycle in $G$ with the required property. 


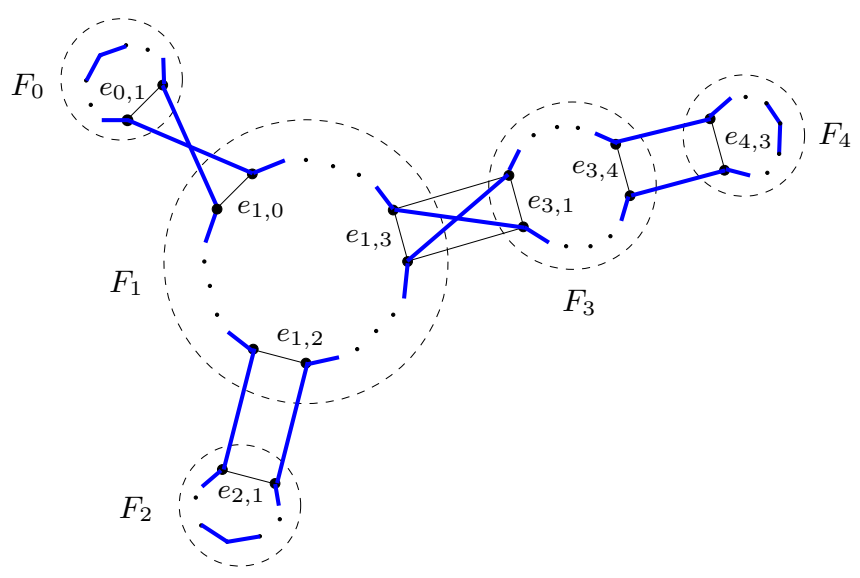

Figure 3: Illustration of Lemma 2.7.

We remark that if $d_{T}\left(f_{i}\right)=1$ for some $i$, then the Hamilton cycle $C$ constructed in Lemma 2.7 contains all except one edge of $E\left(C_{i}\right)$.

\section{2-trees of diameter two}

In this section we characterize 2-trees $H$ of diameter two in which $G_{4}(H)$ has a Hamilton cycle. We begin by defining a class of 2 -trees that we denote by $T(p, q, r)$ (see Figure 4 ).

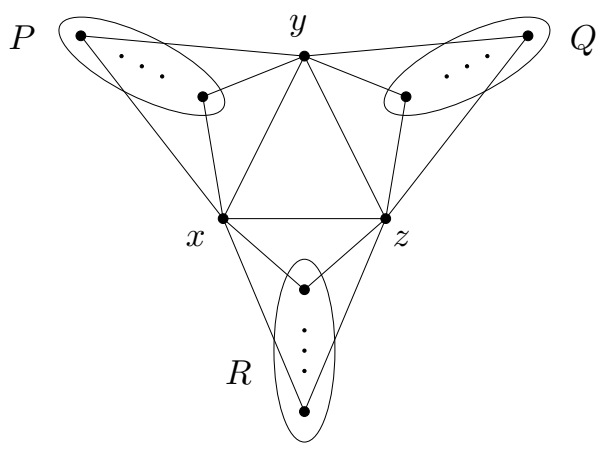

Figure 4: The 2-tree $T(p, q, r)$.

Definition 3.1. Let $P, Q$, and $R$ be pairwise disjoint independent sets of vertices with $|P|:=p,|Q|:=q$, and $|R|:=r$. The graph $T(p, q, r)$ is the graph on $p+q+r+3$ vertices defined on vertex set $\{x, y, z\} \cup P \cup Q \cup R$ where the subgraph induced by $P \cup Q \cup R$ is an independent set, and

- the subgraph induced by $\{x, y\} \cup P$ is isomorphic to $K_{1,1, p}$;

- the subgraph induced by $\{y, z\} \cup Q$ is isomorphic to $K_{1,1, q}$;

- the subgraph induced by $\{z, x\} \cup R$ is isomorphic to $K_{1,1, r}$.

A dominating vertex in a graph is a vertex adjacent to all other vertices of the graph. 
Lemma 3.2. A graph $H$ is a 2-tree of diameter two if and only if $H$ has a dominating vertex or $H \cong T(p, q, r)$ for $p, q, r>0$.

Proof. Any 2-tree with a dominating vertex has diameter two, and one can easily verify that $T(p, q, r)$ is a 2-tree of diameter two for any $p, q, r>0$.

For the converse, suppose $H$ is a 2-tree of diameter two. We proceed by induction on $n:=|V(H)|$. When $n=4, H$ is isomorphic to the graph obtained from $K_{4}$ by deleting one edge, and has a dominating vertex. Now suppose that $n \geq 5$, and let $u \in V(H)$ be a leaf of $H$, i.e., $d_{H}(u)=2$. By the induction hypothesis, $H-u$ has a dominating vertex, or $H-u \cong T\left(p^{\prime}, q^{\prime}, r^{\prime}\right)$ for some $p^{\prime}, q^{\prime}, r^{\prime}>0$. If $H-u \cong T\left(p^{\prime}, q^{\prime}, r^{\prime}\right)$ for some $p^{\prime}, q^{\prime}, r^{\prime}>0$, then let $V(H-u):=\{x, y, z\} \cup P^{\prime} \cup Q^{\prime} \cup R^{\prime}$, where $\left|P^{\prime}\right|:=p^{\prime}$, $\left|Q^{\prime}\right|:=q^{\prime}$, and $\left|R^{\prime}\right|:=r^{\prime}$. Since $H$ has diameter two, $u$ must be adjacent to at least two vertices from $\{x, y, z\}$. However $d_{H}(u)=2$, and thus $N_{H}(u)=\{x, y\}, N_{H}(u)=\{y, z\}$, or $N_{H}(u)=\{z, x\}$. It follows that $H \cong T\left(p^{\prime}+1, q^{\prime}, r^{\prime}\right), H \cong T\left(p^{\prime}, q^{\prime}+1, r^{\prime}\right)$, or $H \cong T\left(p^{\prime}, q^{\prime}, r^{\prime}+1\right)$, respectively.

Now suppose $H-u$ has a dominating vertex, $x$. If $u x \in E(H)$, then $x$ is a dominating vertex in $H$. Otherwise, let $y$ and $z$ denote the neighbours of $u$ in $H$, and note that $y z \in$ $E(H)$. Since $H$ has diameter two, every vertex in $V(H-u)$ is adjacent to $x$ and at least one of $y$ or $z$. Let $P^{\prime}$ be the set of vertices in $H-u$ adjacent to both $x$ and $y, R^{\prime}$ be the set of vertices in $H-u$ adjacent to both $x$ and $z$, and suppose $\left|P^{\prime}\right|:=p^{\prime}$ and $\left|R^{\prime}\right|:=r^{\prime}$. Since $H-u$ is a 2-tree, $P^{\prime} \cup R^{\prime}$ is an independent set. Therefore, $P^{\prime} \cup R^{\prime} \cup\{u\}$ is an independent set in $H$, and thus $H \cong T\left(p^{\prime}, 1, r^{\prime}\right)$.

In what follows, we first prove that if $p, q, r>0$, then $G_{4}(T(p, q, r))$ has a Hamilton cycle. Let $G$ be a graph with vertex partition $\left\{F_{0}, F_{1}, \ldots, F_{N-1}\right\}$. For each $i, 1 \leq i \leq$ $N-1$, let $S_{i-1} \subseteq F_{i-1}$ and $S_{i}^{\prime} \subseteq F_{i}$ denote the vertices incident to the edges of $\left[F_{i-1}, F_{i}\right]$.

Lemma 3.3. If $p, q, r>0$, then $G_{4}(T(p, q, r))$ has a Hamilton cycle.

Proof. Let $V\left(K_{3}\right):=\{x, y, z\}$. Suppose $f: V\left(K_{3}\right) \rightarrow\{1,2,3,4\}$ is a 4-colouring of $K_{3}$ and $V\left(G_{4}\left(K_{3}\right)\right):=\left\{f_{0}, f_{1}, \ldots, f_{N-1}\right\}$. Since $G_{4}\left(K_{3}\right)$ has a Hamilton cycle by [6, Theorem 4.1], we may assume that $f_{0} f_{1} \cdots f_{N-1}$ is a Hamilton path in $G_{4}\left(K_{3}\right)$.

For $0 \leq i \leq N-1$, let $F_{i}$ be the set of 4-colourings of $T(p, q, r)$ that agree with $f_{i}$ on $\{x, y, z\}$. In order to simplify notation, we define $G:=G_{4}(T(p, q, r))$. Then $\left\{F_{0}, F_{1}, \ldots, F_{N-1}\right\}$ is a partition of the vertices of $G$, and $G\left[F_{i}\right] \cong Q_{p+q+r}, 0 \leq i \leq$ $N-1$.

Let $s t \in\left[F_{i-1}, F_{i}\right]$, where $s \in S_{i-1}$ and $t \in S_{i}^{\prime}$ for some $1 \leq i \leq N-1$. Then $s(u)=t(u)$ for all $u \in V(G) \backslash\{v\}$, where $v$ is one of $\{x, y, z\}$, and $s(v) \neq t(v)$. Thus, $\left[F_{i-1}, F_{i}\right]$ is a set of independent edges. If $v=x$, then $s(u)=s(w)$ for all $u, w \in P$ and for all $u, w \in R$, and $t(u)=t(w)$ for all $u, w \in P$ and for all $u, w \in R$. Thus $G\left[S_{i-1}\right] \cong$ $Q_{q} \cong G\left[S_{i}^{\prime}\right]$, and $G\left[S_{i-1} \cup S_{i}^{\prime}\right] \cong Q_{q+1}$. Analogously, if $v=y, G\left[S_{i-1}\right] \cong Q_{r} \cong G\left[S_{i}^{\prime}\right]$ and $G\left[S_{i-1} \cup S_{i}^{\prime}\right] \cong Q_{r+1}$; and if $v=z, G\left[S_{i-1}\right] \cong Q_{p} \cong G\left[S_{i}^{\prime}\right], G\left[S_{i-1} \cup S_{i}^{\prime}\right] \cong Q_{p+1}$.

Consider the path $f_{i-1} f_{i} f_{i+1}$ in $G_{4}\left(K_{3}\right), 1 \leq i \leq N-2$. Note that

$$
f_{i-1}(x), f_{i}(x), f_{i+1}(x)
$$

use at most two different colours; otherwise, there would be only one colour available for $y$ and $z$, which is impossible since $y$ and $z$ always receive different colours. Analogously, $f_{i-1}, f_{i}, f_{i+1}$ assign at most two different colours to each of $y$ and $z$. It follows that if $\left|\left[F_{i-1}, F_{i}\right]\right|=\left|\left[F_{i}, F_{i+1}\right]\right|=2$, then $\left[F_{i-1}, F_{i}\right] \cup\left[F_{i}, F_{i+1}\right] \not 2 P_{3}$. 
For each edge $f_{i-1} f_{i}$ with $1 \leq i \leq N-1$, we choose a 4-cycle, $B_{i-1}$, containing exactly two edges of $\left[F_{i-1}, F_{i}\right]$ as follows.

- For each $\left|\left[F_{i-1}, F_{i}\right]\right|=2, G\left[S_{i-1} \cup S_{i}^{\prime}\right]$ is a 2 -cube, so we choose $B_{i-1}:=G\left[S_{i-1} \cup\right.$ $\left.S_{i}^{\prime}\right]=a_{i-1} c_{i-1} d_{i} b_{i} a_{i-1}$, where $a_{i-1}, c_{i-1} \in F_{i-1}$ and $b_{i}, d_{i} \in F_{i}$.

- For $i=1,2, \ldots, N-1$, and $\left|\left[F_{i-1}, F_{i}\right]\right|>2$, first note that $\left|\left[F_{i-1}, F_{i}\right]\right| \geq 4$ since $G\left[S_{i-1} \cup S_{i}^{\prime}\right] \cong Q_{n}$ for some $n \geq 3$. Thus it is possible to choose edges $a_{i-1} b_{i}$ and $c_{i-1} d_{i}$ of $\left[F_{i-1}, F_{i}\right]$ so that $B_{i-1}:=a_{i-1} c_{i-1} d_{i} b_{i} a_{i-1}$ is edge disjoint from $B_{i-2}$, and also edge disjoint from $B_{i}$ if $\left|\left[F_{i}, F_{i+1}\right]\right|=2$ and $i<N-1$.

Let $e_{i, i+1}:=G\left[S_{i}\right] \cap B_{i}$ and $e_{i+1, i}:=G\left[S_{i+1}^{\prime}\right] \cap B_{i}, 0 \leq i \leq N-2$. Observe that $G$ and the path $f_{0} f_{1} \cdots f_{N-1}$ satisfy conditions (i) and (ii) of Lemma 2.7. Recall that $G\left[F_{i}\right] \cong Q_{p+q+r}, 0 \leq i \leq N-1$, and $p+q+r \geq 3$. Since any pair of edges of $Q_{n}, n \geq 2$, is contained in a Hamilton cycle (see [7, Theorem 4.1]), there exist Hamilton cycles $C_{0}$ in $G\left[F_{0}\right]$ containing $e_{0,1}, C_{N-1}$ in $G\left[F_{N-1}\right]$ containing $e_{N-1, N-2}$, and, for $1 \leq i \leq N-2$, $C_{i}$ in $G\left[F_{i}\right]$ containing $e_{i, i-1}$ and $e_{i, i+1}$. Therefore, by Lemma 2.7, $G$ has a Hamilton cycle.

In the case where $p>0$ and $q=r=0, T(p, q, r)$ has a dominating vertex, and is isomorphic to $K_{2} \vee \bar{K}_{n}$.

Lemma 3.4. For $n \geq 2, G_{4}\left(K_{2} \vee \bar{K}_{n}\right)$ has no Hamilton cycle.

Proof. Let $H:=K_{2} \vee \bar{K}_{n}$ for $n \geq 2$, let $\mathcal{H}:=G_{4}(H)$, and let $u, v$ be the two vertices of $H$ of degree $n+1$. For each $1 \leq i \neq j \leq 4$ let

$$
V_{i j}:=\{c \in V(\mathcal{H}) \mid c(u)=i \text { and } c(v)=j\} .
$$

Then

$$
\left\{V_{12}, V_{13}, V_{14}, V_{21}, V_{23}, V_{24}, V_{31}, V_{32}, V_{34}, V_{41}, V_{42}, V_{43}\right\}
$$

is a partition of $V(\mathcal{H})$. Note that $\left[V_{\alpha \beta}, V_{\gamma \delta}\right] \neq \emptyset$ if and only if $\alpha=\gamma$ or $\beta=\delta$. For $1 \leq$ $i \neq j \leq 4$, let $L_{i j}$ be an assignment of lists to the vertices of $H$ such that $L_{i j}(u):=\{i\}$, $L_{i j}(v):=\{j\}$ and $L_{i j}(x):=\{1,2,3,4\}$ for $x \in V(H-\{u, v\})$. Note that $G_{L_{i j}}(H) \cong$ $\mathcal{H}\left[V_{i j}\right] \cong Q_{n}$, for each $1 \leq i \neq j \leq 4$. Define the three-coloured vertices of $\mathcal{H}$ (that is, the colourings of $H$ with three colours) by $c_{i j k} \in V_{i j}$ such that

$$
c_{i j k}(x):= \begin{cases}i, & \text { if } x=u, \\ j, & \text { if } x=v, \\ k, & \text { otherwise. }\end{cases}
$$

Each $V_{i j}$ has two such vertices, $c_{i j k_{1}}$ and $c_{i j k_{2}}$, where $k_{1}, k_{2} \in\{1,2,3,4\} \backslash\{i, j\}$. Furthermore, $\mathcal{H}-\left\{c_{i j k_{1}}, c_{i j k_{2}}\right\}$ is disconnected so that any Hamilton cycle in $\mathcal{H}$ must contain the edges of a Hamilton path of $\mathcal{H}\left[V_{i j}\right]$ with endpoints $c_{i j k_{1}}$ and $c_{i j k_{2}}$, for each $1 \leq i \neq j \leq 4$.

By [6, Lemma 2.1] there is no Hamilton path in the $n$-dimensional cube from $00 \cdots 0$ to $11 \cdots 1$ whenever $n$ is even. Thus, for $n$ even, there is no Hamilton path in $\mathcal{H}\left[V_{i j}\right]$ with endpoints $c_{i j k_{1}}$ and $c_{i j k_{2}}$. Therefore, $\mathcal{H}$ has no Hamilton cycle when $n$ is even.

For $n$ odd, such Hamilton paths exist and must be used in any Hamilton cycle of $\mathcal{H}$, if one exists. We construct an auxiliary graph $A$ (see Figure 5(a)) where the vertex $(i, j)$ 
represents a Hamilton path in $\mathcal{H}\left[V_{i j}\right]$ from $c_{i j k_{1}}$ to $c_{i j k_{2}}$. There is an edge in $A$ between $\left(i_{1}, j_{1}\right)$ and $\left(i_{2}, j_{2}\right)$ whenever $c_{i_{1} j_{1} k}$ is adjacent to $c_{i_{2} j_{2} k}$ in $\mathcal{H}$. We label the edge between $\left(i_{1}, j_{1}\right)$ and $\left(i_{2}, j_{2}\right)$ by the unique element of $\{1,2,3,4\} \backslash\left(\left\{i_{1}, j_{1}\right\} \cup\left\{i_{2}, j_{2}\right\}\right)$ (see Figure 5(b)). Notice that the edges labelled $i, 1 \leq i \leq 4$, induce a 6 -cycle, and the edges of these four 6 -cycles partition $E(A)$.

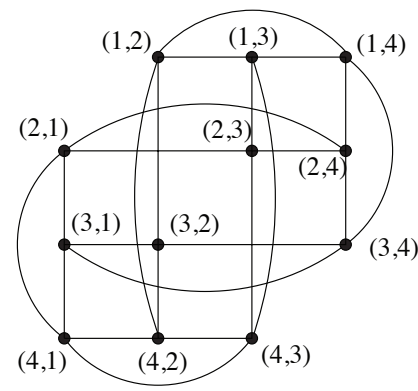

(a)

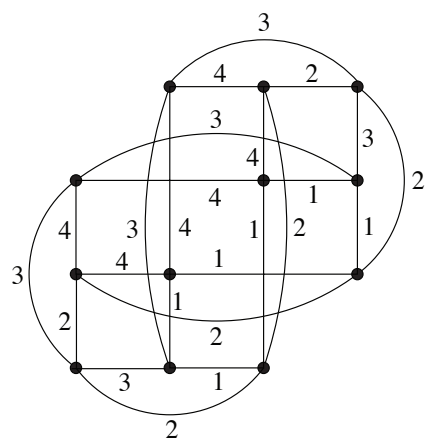

(b)

Figure 5: The auxiliary graph $A$.

A Hamilton cycle in $\mathcal{H}$ corresponds to a Hamilton cycle, $\mathcal{C}$, in $A$ in which any two consecutive edges of $\mathcal{C}$ have different labels. Such a cycle $\mathcal{C}$ uses a matching of size three from each labelled 6 -cycle in $A$. Without loss of generality, we may assume $\mathcal{C}$ contains horizontal edges of the 6 -cycle labelled 1 . Now, $\mathcal{C}$ uses horizontal edges from one of the remaining labelled 6 -cycles, otherwise, $\mathcal{C}$ contains a $K_{3}$. Regardless of whether $\mathcal{C}$ uses horizontal edges of the 6 -cycle labelled by 2,3 or 4 , using vertical edges of the two remaining 6 -cycles gives $\mathcal{C} \cong C_{4} \cup C_{8}$, a contradiction. Therefore $\mathcal{H}$ has no Hamilton cycle.

Observe that if $H$ is a 2 -tree of diameter two having a dominating vertex $u$, then $H \cong$ $T \vee\{u\}$ for some tree $T$.

Lemma 3.5. Let $T$ be a tree on at least two vertices. Then $G_{4}(T \vee\{u\})$ has a Hamilton cycle unless $T$ is a star on at least three vertices or the bipartition of $V(T)$ has two even parts.

The proof of this lemma requires a result that we state here, but whose proof is technical and is postponed to the Appendix A.

Lemma 3.6. Let $T$ be a tree with bipartition $(A, B)$, where $|A|:=\ell$ and $|B|:=r$, and let $G_{3}(T)$ be the 3-colouring graph of $T$ with colours $C=\{1,2,3\}$. Define $c_{i j}$ to be the vertex of $G_{3}(T)$ with $c_{i j}(a)=i$ for all $a \in A$ and $c_{i j}(b)=j$ for all $b \in B$.

(1) If $\ell, r>0$ are both even, then $G_{3}(T)$ has no spanning subgraph consisting only of paths whose ends are in $\left\{c_{12}, c_{13}, c_{21}, c_{23}, c_{31}, c_{32}\right\}$.

(2) If $\ell>1$ is odd and $r>0$ is even, then $G_{3}(T)$ has a Hamilton path from $c_{12}$ to $c_{23}$.

(3) If $\ell>1$ and $r>1$ are both odd, then $G_{3}(T)$ has a Hamilton path from $c_{12}$ to $c_{13}$. 
Proof of Lemma 3.5. Let $T$ be a tree with $|V(T)| \geq 2$ and bipartition $(A, B)$, where $A:=$ $\left\{x_{1}, x_{2}, \ldots, x_{\ell}\right\}$ and $B:=\left\{y_{1}, y_{2}, \ldots, y_{r}\right\}$. Let $H:=T \vee\{u\}$, and let $\mathcal{H}:=G_{4}(H)$ be the 4-colouring graph of $H$ with colours $\{1,2,3,4\}$. For each $1 \leq k \leq 4$, define

$$
V_{k}:=\{c \in V(\mathcal{H}) \mid c(u)=k\}
$$

Then $\left\{V_{1}, V_{2}, V_{3}, V_{4}\right\}$ is a partition of $V(\mathcal{H})$. Define $L_{k}$ to be an assignment of lists with $L_{k}(u):=\{k\}$ and $L_{k}(w):=\{1,2,3,4\}$ for $w \in V(T)$. Note that $G_{L_{k}}(H) \cong \mathcal{H}\left[V_{k}\right] \cong$ $G_{3}(T)$. Define the three-coloured vertices of $\mathcal{H}$ (that is, the colourings of $H$ with three colours) by $c_{i j k} \in V_{k}$ so that $c_{i j k}(x):=i$ for all $x \in A, c_{i j k}(y):=j$ for all $y \in B$.

Observe

$$
\begin{array}{lll}
{\left[V_{1}, V_{2}\right]=\left\{c_{341} c_{342}, c_{431} c_{432}\right\},} & {\left[V_{1}, V_{3}\right]=\left\{c_{241} c_{243}, c_{421} c_{423}\right\},} \\
{\left[V_{1}, V_{4}\right]=\left\{c_{231} c_{234}, c_{321} c_{324}\right\},} & {\left[V_{2}, V_{3}\right]=\left\{c_{142} c_{143}, c_{412} c_{413}\right\},} \\
{\left[V_{2}, V_{4}\right]=\left\{c_{132} c_{134}, c_{312} c_{314}\right\},} & {\left[V_{3}, V_{4}\right]=\left\{c_{123} c_{124}, c_{213} c_{214}\right\} .}
\end{array}
$$

Case 1. Suppose $|V(T)|=2$. Then $T \vee\{u\} \cong K_{3}$ and $G_{4}\left(K_{3}\right)$ has a Hamilton cycle by [6, Theorem 4.1].

Case 2. Suppose $T$ is a star with $|V(T)| \geq 3$. Then $G_{4}(T \vee\{u\}) \cong K_{2} \vee \bar{K}_{n}$ for some $n \geq 2$. By Lemma 3.4, $G_{4}(T \vee\{u\})$ has no Hamilton cycle.

Case 3. Suppose both $|A|$ and $|B|$ are odd. By Lemma 3.6, there is a Hamilton path in $\mathcal{H}\left[V_{1}\right]$ from $c_{421}$ to $c_{431}$, in $\mathcal{H}\left[V_{2}\right]$ from $c_{432}$ to $c_{132}$, in $\mathcal{H}\left[V_{3}\right]$ from $c_{134}$ to $c_{124}$ and in $\mathcal{H}\left[V_{4}\right]$ from $c_{123}$ to $c_{423}$. The union of these paths with edges $\left\{c_{431} c_{432}, c_{132} c_{134}, c_{124} c_{123}\right.$, $\left.c_{423} c_{421}\right\}$ gives a Hamilton cycle in $\mathcal{H}$.

Case 4. Suppose one of $|A|$ and $|B|$ is even and the other is odd. Without loss of generality, $|A|$ is odd and $|B|$ is even. By Lemma 3.6, there is a Hamilton path in $\mathcal{H}\left[V_{1}\right]$ from $c_{231}$ to $c_{341}$, in $\mathcal{H}\left[V_{2}\right]$ from $c_{342}$ to $c_{412}$, in $\mathcal{H}\left[V_{3}\right]$ from $c_{413}$ to $c_{123}$ and in $\mathcal{H}\left[V_{4}\right]$ from $c_{124}$ to $c_{234}$. The union of these paths with edges $\left\{c_{341} c_{342}, c_{412} c_{413}, c_{123} c_{124}, c_{234} c_{231}\right\}$ gives a Hamilton cycle in $\mathcal{H}$.

Case 5. Suppose both $|A|$ and $|B|$ are even, and suppose $\mathcal{H}$ contains a Hamilton cycle $\mathcal{C}$. Then $\mathcal{C}\left[V_{1}\right]$ is a spanning subgraph of $\mathcal{H}\left[V_{1}\right] \cong G_{3}(T)$ consisting of a union of paths whose endpoints are three-coloured vertices of $V_{1}$, contradicting Lemma 3.6. Thus in this case, $\mathcal{H}$ has no Hamilton cycle.

\section{Constructing 2-trees of diameter at least three}

To complete the proof of our main result, we must show that if $H^{\prime}$ is a 2-tree with diameter at least three, then $k_{0}\left(H^{\prime}\right)=4$. Naively deleting two leaves with the intention of applying Remark 2.6 may be problematic. For example, let $H^{\prime}$ be a 2-tree with diameter at least three having leaves $x$ and $y$ of distance at least three. Let $H=H^{\prime}-\{x, y\}, N_{H^{\prime}}(x)=$ $\left\{x_{1}, x_{2}\right\}$, and suppose $G_{4}(H)$ has a Hamilton cycle $f_{0} f_{1} \cdots f_{N-1} f_{0}$. Let $F_{i}$ be the set of 4-colourings of $H^{\prime}$ that agree with $f_{i}$ on $V(H), 0 \leq i \leq N-1$. In the case that $f_{i-1} f_{i}$ arises from a colour change on $x_{1}$ and $f_{i} f_{i+1}$ arises from a colour change on $x_{2}$, the subgraph $G\left[F_{i-1} \cup F_{i} \cup F_{i+1}\right]$ is isomorphic to the forbidden subgraph in Figure 2. Because of this we take a more general approach. Suppose a 2-tree $H^{\prime}$ is obtained from a 2-tree $H$ by repeatedly adding vertices of degree two. Instead of a Hamilton cycle in $G_{4}(H)$ we take 
a spanning tree satisfying certain properties providing the flexibility needed to construct a Hamilton cycle in $G_{4}\left(H^{\prime}\right)$. Our approach does not require $G_{4}(H)$ to have a Hamilton cycle. To facilitate this procedure, we define nine operations (see Figure 6).

Definition 4.1. Let $H$ be a 2-tree. Then $H^{\prime}$ is obtained from $H$ by

Operation I if $\{\alpha, \beta, \gamma\}:=V\left(H^{\prime}\right) \backslash V(H),\left\{a_{1} a_{2}, b_{1} b_{2}, c_{1} c_{2}\right\} \subseteq E(H)$, and $N_{H^{\prime}}(\alpha)=\left\{a_{1}, a_{2}\right\}, N_{H^{\prime}}(\beta)=\left\{b_{1}, b_{2}\right\}, N_{H^{\prime}}(\gamma)=\left\{c_{1}, c_{2}\right\}$.

Operation II if $\{\alpha, \beta, \gamma\}:=V\left(H^{\prime}\right) \backslash V(H),\left\{x a, x b, c_{1} c_{2}\right\} \subseteq E(H)$, and $N_{H^{\prime}}(\alpha)=$ $\{x, a\}, N_{H^{\prime}}(\beta)=\{x, b\}, N_{H^{\prime}}(\gamma)=\left\{c_{1}, c_{2}\right\}$.

Operation III if $\{\alpha, \beta, \gamma\}:=V\left(H^{\prime}\right) \backslash V(H),\{a x, x y, y c\} \subseteq E(H)$, and $N_{H^{\prime}}(\alpha)=$ $\{a, x\}, N_{H^{\prime}}(\beta)=\{x, y\}, N_{H^{\prime}}(\gamma)=\{c, y\}$.

Operation IV if $\{\alpha, \beta, \gamma, \delta\}:=V\left(H^{\prime}\right) \backslash V(H),\{x y, z w\} \subseteq E(H)$, and $N_{H^{\prime}}(\alpha)=$ $N_{H^{\prime}}(\beta)=\{x, y\}, N_{H^{\prime}}(\gamma)=N_{H^{\prime}}(\delta)=\{w, z\}$.

Operation $\mathbf{V}$ if $\{\alpha, \beta, \gamma, \delta\}:=V\left(H^{\prime}\right) \backslash V(H),\{x y, z w\} \subseteq E(H)$, and $N_{H^{\prime}}(\alpha)=$ $\{\beta, x, y\}, N_{H^{\prime}}(\beta)=\{\alpha, y\}, N_{H^{\prime}}(\gamma)=N_{H^{\prime}}(\delta)=\{w, z\}$.

Operation VI if $\{\alpha, \beta, \gamma, \delta\}:=V\left(H^{\prime}\right) \backslash V(H),\{x y, z w\} \subseteq E(H)$, and $N_{H^{\prime}}(\alpha)=$ $\{\beta, x, y\}, N_{H^{\prime}}(\beta)=\{\alpha, y\}, N_{H^{\prime}}(\gamma)=\{\delta, w, z\}, N_{H^{\prime}}(\delta)=\{\gamma, z\}$.

Operation VII if $\{\alpha, \beta, \gamma, \delta\}:=V\left(H^{\prime}\right) \backslash V(H),\{x y, x z\} \subseteq E(H)$, and $N_{H^{\prime}}(\alpha)=$ $\{\beta, x, y\}, N_{H^{\prime}}(\beta)=\{\alpha, y\}, N_{H^{\prime}}(\gamma)=\{\delta, x, z\}, N_{H^{\prime}}(\delta)=\{\gamma, z\}$.

Operation VIII if $\{\alpha, \beta, \gamma, \delta\}:=V\left(H^{\prime}\right) \backslash V(H),\{x y, x z\} \subseteq E(H)$, and $N_{H^{\prime}}(\alpha)=$ $\{\beta, x, y\}, N_{H^{\prime}}(\beta)=\{x, \alpha\}, N_{H^{\prime}}(\gamma)=\{\delta, x, z\}, N_{H^{\prime}}(\delta)=\{\gamma, z\}$.

Operation IX if $\{\alpha, \beta, \gamma, \delta\}:=V\left(H^{\prime}\right) \backslash V(H),\{x y, x z\} \subseteq E(H)$, and $N_{H^{\prime}}(\alpha)=$ $\{\beta, x, y\}, N_{H^{\prime}}(\beta)=\{\alpha, y\}, N_{H^{\prime}}(\gamma)=N_{H^{\prime}}(\delta)=\{x, z\}$.

Remark 4.2. Since each of Operations I through IX can be performed by sequentially adding simplicial vertices of degree two to $H, H^{\prime}$ is a 2 -tree.

Recall that $D$ denotes the unique 2-tree of diameter three on six vertices (Figure 1(a)).

Theorem 4.3. A graph $H^{\prime}$ is a 2-tree of diameter at least three if and only if $H^{\prime} \cong D$ or $H^{\prime}$ can be obtained from a 2-tree $H$ by applying one of Operations I through IX.

Proof. $(\Leftarrow)$ : If $H^{\prime} \cong D$, the result is trivially true. Otherwise, it follows from Remark 4.2 that $H^{\prime}$ is a 2 -tree. Since each operation produces two leaves that are distance at least three apart, $H^{\prime}$ has diameter at least three.

$(\Rightarrow)$ : As already observed, $D$ is the unique 2 -tree of diameter three on six vertices. The 2-trees on three, four and five vertices all have diameter less than three. Thus, we may assume that $H^{\prime}$ is a 2 -tree of diameter three and $\left|V\left(H^{\prime}\right)\right| \geq 7$. Since $H^{\prime}$ has diameter at least three, there are at least two leaves whose neighbourhoods are vertex disjoint. We consider cases based on the number of edges induced by the neighbourhoods of the leaves of $H^{\prime}$, and the number of leaves of $H^{\prime}$.

Case 1. First assume that the neighbourhoods of the leaves of $H^{\prime}$ induce at least three distinct edges. 


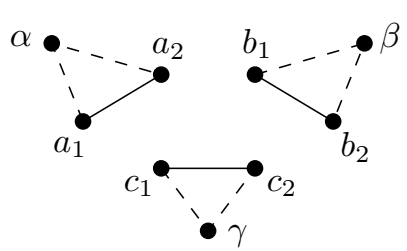

(a) Operation I

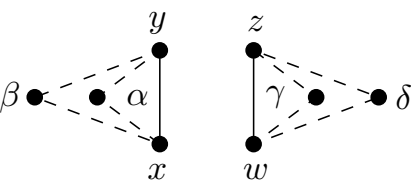

(d) Operation IV

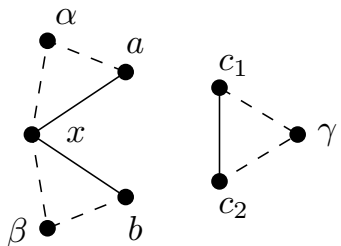

(b) Operation II

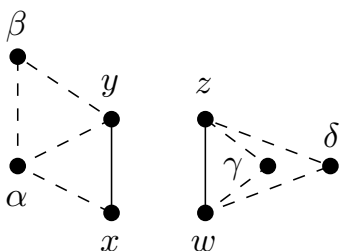

(e) Operation V

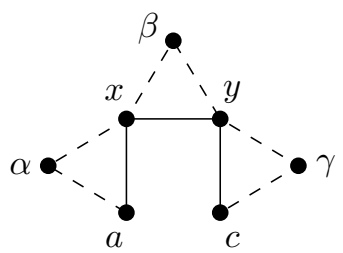

(c) Operation III

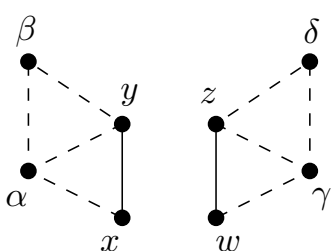

(f) Operation VI

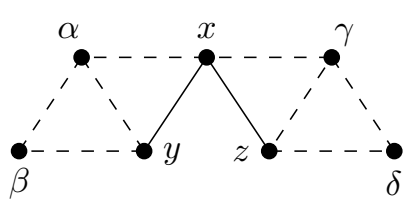

(g) Operation VII

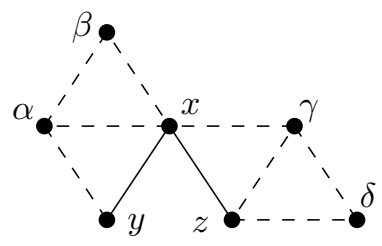

(h) Operation VIII

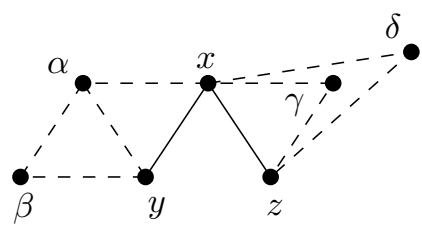

(i) Operation IX

Figure 6: Operations I through IX applied to a graph $H$ by the addition of vertices $\alpha, \beta, \gamma$, and $\delta$ where applicable. The solid edges belong to $H$ and the dotted edges are added to construct $H^{\prime}$.

If $H^{\prime}$ has three leaves whose neighbourhoods are pairwise vertex disjoint, then $H^{\prime}$ contains vertex disjoint edges $a_{1} a_{2}, b_{1} b_{2}, c_{1} c_{2}$, and leaves $\alpha, \beta, \gamma$ with $N(\alpha)=\left\{a_{1}, a_{2}\right\}$, $N(\beta)=\left\{b_{1}, b_{2}\right\}, N(\gamma)=\left\{c_{1}, c_{2}\right\}$. Letting $H:=H^{\prime}-\{\alpha, \beta, \gamma\}$ results in a 2-tree, and applying Operation I to $H$ produces $H^{\prime}$.

We may now assume that no three leaves of $H^{\prime}$ have neighbourhoods that are pairwise vertex disjoint. Choose two leaves $\alpha$ and $\gamma$ whose neighbourhoods are vertex disjoint, and let $\beta \notin\{\alpha, \gamma\}$ be a leaf such that $N(\beta) \notin\{N(\alpha), N(\gamma)\}$. If $N(\beta)$ intersects exactly one of $N(\alpha)$ and $N(\gamma)$, then we may assume without loss of generality that $|N(\beta) \cap N(\alpha)|=1$ and $N(\beta) \cap N(\gamma)=\emptyset$. Then $H^{\prime}$ contains a path of length two, axb and an edge $c_{1} c_{2}$ that is vertex disjoint from $a x b$, such that $N(\alpha)=\{a, x\}, N(\beta)=\{x, b\}$, and $N(\gamma)=\left\{c_{1}, c_{2}\right\}$. Letting $H:=H^{\prime}-\{\alpha, \beta, \gamma\}$ results in a 2-tree, and applying Operation II to $H$ produces $H^{\prime}$. Otherwise, $|N(\beta) \cap N(\alpha)|=|N(\beta) \cap N(\gamma)|=1$, and $H^{\prime}$ contains a path of length three, $c_{1} x y c_{2}$ such that $N(\alpha)=\left\{c_{1}, x\right\}, N(\beta)=\{x, y\}$ and $N(\gamma)=\left\{y, c_{2}\right\}$. Letting $H:=H^{\prime}-\{\alpha, \beta, \gamma\}$ results in a 2-tree, and applying Operation III to $H$ produces $H^{\prime}$.

Case 2. We may now assume that the neighbourhoods of the leaves of $H^{\prime}$ induce exactly two edges. 
Case 2(a). Suppose that $H^{\prime}$ has at least three leaves. Let leaves $\beta$ and $\gamma$ have neighbourhoods that are vertex disjoint, and let $\delta \notin\{\beta, \gamma\}$ be a leaf. Without loss of generality, $N(\delta)=N(\gamma)$.

If there exists a leaf $\alpha$ with $N(\alpha)=N(\beta)$, then $H:=H^{\prime}-\{\alpha, \beta, \gamma, \delta\}$ is a 2-tree, and applying Operation IV to $H$ produces $H^{\prime}$. Otherwise, no other leaf of $H^{\prime}$ has the same neighbourhood as $\beta$. If we let $N(\beta):=\{\alpha, y\}$, then at least one of $\{\alpha, y\}$ is a leaf of $H^{\prime}-\beta$; without loss of generality, $\alpha$ is a leaf of $H^{\prime}-\beta$, and so $d(\alpha)=3$. It follows that $N(\alpha)=\{\beta, y, x\}$ for some $x \notin\{\alpha, \beta, \gamma, \delta, y\}$, and that $x y \in E\left(H^{\prime}\right)$.

Let $N(\gamma)=N(\delta)=\{w, z\}$. We consider two cases depending on $|\{x\} \cap\{w, z\}|$. If $|\{x\} \cap\{w, z\}|=0$, then $H:=H^{\prime}-\{\alpha, \beta, \gamma, \delta\}$ is a 2-tree, and applying Operation $\mathrm{V}$ to $H$ produces $H^{\prime}$. If $|\{x\} \cap\{w, z\}|=1$, then $y=w$ or $y=z$, and the two cases are analogous. The graph $H:=H^{\prime}-\{\alpha, \beta, \gamma, \delta\}$ is a 2-tree, and applying Operation IX to $H$ produces $H^{\prime}$.

Case 2(b). Finally, assume that $H^{\prime}$ has exactly two leaves, $\beta$ and $\delta$, with $N(\beta)=\{\alpha, y\}$ and $N(\delta)=\{\gamma, z\}$. Since $d_{H^{\prime}}(\beta, \delta) \geq 3,\{\alpha, y\} \cap\{\gamma, z\}=\emptyset$. We may assume, without loss of generality, that $\alpha$ and $\gamma$ are leaves in $H^{\prime}-\{\beta, \delta\}$, and so $d(\alpha)=3$ and $d(\gamma)=3$. It follows that $N(\alpha)=\{\beta, y, p\}$ and $N(\gamma)=\{\delta, z, q\}$ for some $p, q \notin\{\beta, \alpha, \delta, \gamma\}$ with $p \neq y, q \neq z$ and $p y, q z \in E\left(H^{\prime}\right)$.

We consider three cases depending on $|\{p, y\} \cap\{q, z\}|$. If $|\{p, y\} \cap\{q, z\}|=0$, then $H:=H^{\prime}-\{\alpha, \beta, \gamma, \delta\}$ is a 2-tree, and applying Operation VI to $H$ produces $H^{\prime}$. If $|\{p, y\} \cap\{q, z\}|=1$, then either $p=q, p=z$, or $q=y$. In the case $p=q$, $H:=H^{\prime}-\{\alpha, \beta, \gamma, \delta\}$ is a 2-tree, and applying Operation VII to $H$ produces $H^{\prime}$. In the case $p=z, H:=H^{\prime}-\{\alpha, \beta, \gamma, \delta\}$ is a 2-tree, and applying Operation VIII to $H$ produces $H^{\prime}$. The case $q=y$ is analogous to the case $p=z$. Finally if $|\{p, y\} \cap\{q, z\}|=2$, then the fact that $y \neq z$ implies that $p=z$ and $q=y$, and hence $H^{\prime} \cong D$, contradicting the fact that $\left|V\left(H^{\prime}\right)\right| \geq 7$.

\section{Operations and the 4-colouring graph}

Let $H$ be a 2-tree, and let $H^{\prime}$ be the 2-tree obtained from $H$ by applying one of the Operations I through IX. As before, let $V\left(G_{4}(H)\right):=\left\{f_{0}, f_{1}, \ldots, f_{N-1}\right\}$, and let $F_{j} \subseteq$ $V\left(G_{4}\left(H^{\prime}\right)\right)$ be the set of 4-colourings of $H^{\prime}$ that agree with $f_{j}$ on the vertices of $H$, $0 \leq j \leq N-1$. For each Operation I through IX, what follows is a description of the subgraph of $G:=G_{4}\left(H^{\prime}\right)$ induced by $F_{i}, 0 \leq i \leq N-1$, and also a description of the subgraph of $G$ induced by $F_{i} \cup F_{j}$ when $f_{i} f_{j} \in E\left(G_{4}(H)\right), 0 \leq i, j \leq N-1$. Each edge $f_{i} f_{j}$ of $G_{4}(H)$ is also assigned a label to indicate the structure of $G\left[\bar{F}_{i} \cup F_{j}\right]$. We remark that for a path $f_{i} f_{j} f_{k}$ of length two in $G_{4}(H)$, if $f_{i}(u) \neq f_{j}(u)$ for some $u \in V(H)$, then $f_{j}(u)=f_{k}(u)$.

\subsection{Operation I}

If $H^{\prime}$ is obtained from $H$ using Operation I, then there are two choices of colour for each of the vertices $\alpha, \beta$, and $\gamma$, so for each $i, 0 \leq i \leq N-1, G\left[F_{i}\right] \cong Q_{3}$. To simplify the labelling of the vertices of $G\left[F_{i}\right]$, we label the faces of a plane drawing of $Q_{3}$ as shown in Figure 7(a), where $\alpha_{1}$ and $\alpha_{2}$ are the possible colours of vertex $\alpha, \beta_{1}$ and $\beta_{2}$ are the possible colours of vertex $\beta$, and $\gamma_{1}$ and $\gamma_{2}$ are the possible colours of vertex $\gamma$. Without loss of generality, assume these colour choices are as shown in Figure 7(b). A vertex $u$ of 
$Q_{3}$ is assigned label $\alpha_{i} \beta_{j} \gamma_{k}$ where $i, j, k \in\{1,2\}$, and $u$ is incident with the faces labelled $\alpha_{i}, \beta_{j}$ and $\gamma_{k}$ (see Figure 7(c)).

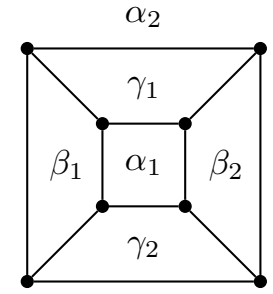

(a)

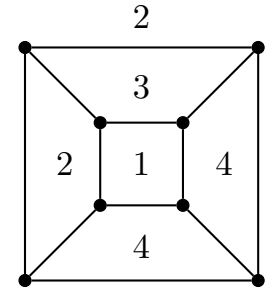

(b)

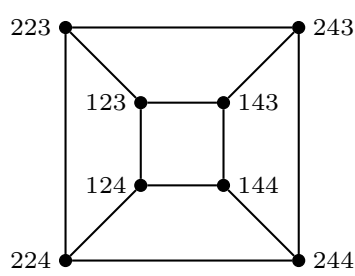

(c)

Figure 7: Labelling $G\left[F_{i}\right]$.

The following arguments can be made with sets of colours $\left\{f_{i}\left(a_{1}\right), f_{i}\left(a_{2}\right)\right\},\left\{f_{i}\left(b_{1}\right)\right.$, $\left.f_{i}\left(b_{2}\right)\right\}$ and $\left\{f_{i}\left(c_{1}\right), f_{i}\left(c_{2}\right)\right\}$ each chosen independently as a subset of $\{1,2,3,4\}$. To ease notation, we assume that $f_{i}\left(a_{1}\right)=3, f_{i}\left(a_{2}\right)=4, f_{i}\left(b_{1}\right)=1, f_{i}\left(b_{2}\right)=3, f_{i}\left(c_{1}\right)=1$, and $f_{i}\left(c_{2}\right)=2$. Then the colour choices for $\alpha$ are $\{1,2\}$, for $\beta$ are $\{2,4\}$, and for $\gamma$ are $\{3,4\}$. As already noted, $G\left[F_{i}\right] \cong Q_{3}$; assume that $G\left[F_{i}\right]$ has been drawn in the plane and labelled as in Figure 8(a).

If $f_{i} f_{j} \in E\left(G_{4}(H)\right)$, then $f_{j}$ is obtained from $f_{i}$ by changing the colour of a single vertex in $V(H)$. We label each edge $f_{i} f_{j} \in E\left(G_{4}(H)\right)$ according to the structure of $G\left[F_{i} \cup F_{j}\right]$.

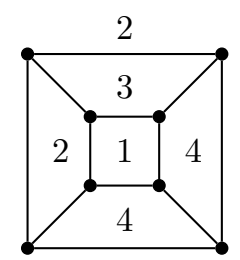

(a)

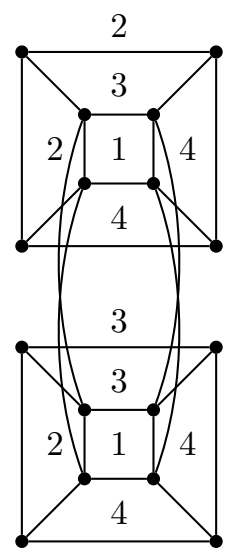

(b)

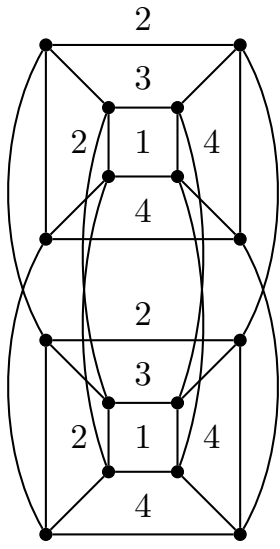

(c)

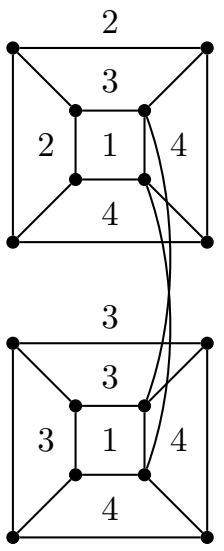

(d)

Figure 8: $G\left[F_{i}\right]$ and $G\left[F_{i} \cup F_{j}\right]$ for Operation I, II and III.

(i) $f_{i}(v) \neq f_{j}(v)$ for $v \in\left\{a_{1}, a_{2}\right\}$. Without loss of generality, suppose $v=a_{1}$. Since $H$ is a 2 -tree, there is a vertex $a_{3} \in V(H)$ such that $H\left[\left\{a_{1}, a_{2}, a_{3}\right\}\right] \cong K_{3}$. Observe $f_{i}\left(a_{3}\right) \in\{1,2\}$; we may assume $f_{i}\left(a_{3}\right)=1$. Then $f_{j}\left(a_{1}\right)=2 .{ }^{1}$ Since $f_{j}\left(a_{2}\right)=$

\footnotetext{
${ }^{1}$ Since $f_{j}\left(a_{1}\right)$ is uniquely determined there is no $f_{\ell}$ with $\ell \neq j$ such that $f_{i}$ and $f_{\ell}$ differ on $a_{1}$.
} 
$f_{i}\left(a_{2}\right)=4$, the colours available for $\alpha$ are $\{1,3\}$; the colours available for $\beta$ and $\gamma$ are unchanged. Therefore $\left[F_{i}, F_{j}\right]$ is a matching of size four between the 4-cycle bounding the $\alpha_{1}$ face in $G\left[F_{i}\right]$ and the 4-cycle bounding either the $\alpha_{1}$ face or the $\alpha_{2}$ face in $G\left[F_{j}\right]$. Thus $G\left[F_{i} \cup F_{j}\right]$ is isomorphic to the graph in Figure 8(b). We label the edge $f_{i} f_{j}$ by a-sq. It follows from Footnote 1 that there are at most two edges incident to $f_{i}$ having label a-sq. Furthermore, we remark that if $f_{i} f_{j_{1}}, f_{i} f_{j_{2}} \in E\left(G_{4}(H)\right)$ both have label a-sq, then the four vertices of $F_{i}$ incident to the edges of $\left[F_{i}, F_{j_{1}}\right]$ are the same four vertices of $F_{i}$ that are incident to the edges of $\left[F_{i}, F_{j_{2}}\right]$, and induce a 4 -cycle in $G\left[F_{i}\right]$ that bounds a face with label $\alpha_{1}$ or $\alpha_{2}$.

(ii) $f_{i}(v) \neq f_{j}(v)$ for $v \in\left\{b_{1}, b_{2}\right\}$. As in (i), we label the edge $f_{i} f_{j}$ by b-sq. Note that there are at most two edges incident to $f_{i}$ having label b-sq. If $f_{i} f_{j_{1}}, f_{i} f_{j_{2}} \in$ $E\left(G_{4}(H)\right)$ both have label b-sq, then the four vertices of $F_{i}$ incident to the edges of $\left[F_{i}, F_{j_{1}}\right]$ are the same four vertices of $F_{i}$ that are incident to the edges of $\left[F_{i}, F_{j_{2}}\right]$, and induce a 4 -cycle in $G\left[F_{i}\right]$ that bounds a face with label $\beta_{1}$ or $\beta_{2}$.

(iii) $f_{i}(v) \neq f_{j}(v)$ for $v \in\left\{c_{1}, c_{2}\right\}$. As in (i) and (ii), we label the edge $f_{i} f_{j}$ by c-sq. Note that there are at most two edges incident to $f_{i}$ having label c-sq. If $f_{i} f_{j_{1}}, f_{i} f_{j_{2}} \in E\left(G_{4}(H)\right)$ both have label c-sq, then the four vertices of $F_{i}$ incident to the edges of $\left[F_{i}, F_{j_{1}}\right]$ are the same four vertices of $F_{i}$ that are incident to the edges of $\left[F_{i}, F_{j_{2}}\right]$, and induce a 4 -cycle in $G\left[F_{i}\right]$ that bounds a face with label $\gamma_{1}$ or $\gamma_{2}$.

(iv) $f_{i}(u) \neq f_{j}(u)$ for $u \in V(H) \backslash\left\{a_{1}, a_{2}, b_{1}, b_{2}, c_{1}, c_{2}\right\}$. In this case, the vertex labels on $G\left[F_{i}\right]$ and $G\left[F_{j}\right]$ are identical. Thus $\left[F_{i}, F_{j}\right]$ is a perfect matching, and $G\left[F_{i} \cup F_{j}\right]$ is isomorphic to the graph in Figure 8(c). We label the edge $f_{i} f_{j}$ by pm.

Table 1: Summary of Operation I.

\begin{tabular}{c|c|c} 
& Subgraph induced by & Label of $f_{i} f_{j}$ \\
\hline \hline Vertex whose colour is changed & $F_{i} \cup F_{j}$ & a-sq \\
\hline$a_{1}, a_{2}$ & Figure 8(b) & b-sq \\
\hline$b_{1}, b_{2}$ & Figure 8(b) & c-sq \\
\hline$c_{1}, c_{2}$ & Figure 8(b) & pm
\end{tabular}

\subsection{Operation II}

As with Operation I, $G\left[F_{i}\right] \cong Q_{3}$ for each $i, 0 \leq i \leq N-1$. We may assume that $f_{i}(a)=4, f_{i}(x)=3, f_{i}(b)=1, f_{i}\left(c_{1}\right)=1$, and $f_{i}\left(c_{2}\right)=2$. Then the colour choices for $\alpha$ are $\{1,2\}$, for $\beta$ are $\{2,4\}$, and for $\gamma$ are $\{3,4\}$. Using the same labelling convention as for Operation I, we assume that $G\left[F_{i}\right]$ is drawn in the plane and labelled as in Figure 8(a).

If $f_{i} f_{j} \in E\left(G_{4}(H)\right)$, then $f_{j}$ is obtained from $f_{i}$ by changing the colour of a single vertex in $V(H)$. We label each edge $f_{i} f_{j} \in E\left(G_{4}(H)\right)$ according to the structure of $G\left[F_{i} \cup F_{j}\right]$.

(i) $f_{i}(v) \neq f_{j}(v)$ for $v \in\left\{a, b, c_{1}, c_{2}\right\}$. This is analogous to Operation I when the colour of one of $\left\{a_{1}, a_{2}, b_{1}, b_{2}, c_{1}, c_{2}\right\}$ is changed, and thus $G\left[F_{i} \cup F_{j}\right]$ is isomorphic 
to the graph in Figure 8(b). We label the edge $f_{i} f_{j}$ by either a-sq, b-sq or c-sq as in Operation I.

(ii) $f_{i}(x) \neq f_{j}(x)$. We may assume that $f_{j}(x)=2$. Then the colours available for $\alpha$ are $\{1,3\}$ and the colours available for $\beta$ are $\{3,4\}$; the colours available for $\gamma$ are unchanged. Therefore, $\left[F_{i}, F_{j}\right]$ is a matching of size two where $G\left[F_{i}\right]$ and $G\left[F_{j}\right]$ are edges whose endpoint labels are unchanged, and thus $G\left[F_{i} \cup F_{j}\right]$ is isomorphic to the graph in Figure 8(d). We label the edge $f_{i} f_{j}$ by e.

(iii) $f_{i}(u) \neq f_{j}(u)$ for $u \in V(H) \backslash\left\{a, b, x, c_{1}, c_{2}\right\}$. In this case, the vertex labels on $G\left[F_{i}\right]$ and $G\left[F_{j}\right]$ are identical. Thus $\left[F_{i}, F_{j}\right]$ is a perfect matching, and $G\left[F_{i} \cup F_{j}\right]$ is isomorphic to the graph in Figure $8(\mathrm{c})$. We label the edge $f_{i} f_{j}$ by pm.

Table 2: Summary of Operation II.

\begin{tabular}{c|c|c} 
& Subgraph induced by & \\
Vertex whose colour is changed & $F_{i} \cup F_{j}$ & Label of $f_{i} f_{j}$ \\
\hline \hline$a$ & Figure 8(b) & a-sq \\
\hline$b$ & Figure 8(b) & b-sq \\
\hline$c_{1}, c_{2}$ & Figure 8(b) & c-sq \\
\hline$x$ & Figure 8(d) & e \\
\hline$u \in V(H) \backslash\left\{a, b, x, c_{1}, c_{2}\right\}$ & Figure 8(c) & pm
\end{tabular}

\subsection{Operation III}

As with Operations I and II, $G\left[F_{i}\right] \cong Q_{3}$ for each $i, 0 \leq i \leq N-1$. We may assume that $f_{i}(x)=3, f_{i}(y)=1, f_{i}(a)=4$, and $f_{i}(c)=2$. Then the colour choices for $\alpha$ are $\{1,2\}$, for $\beta$ are $\{2,4\}$, and for $\gamma$ are $\{3,4\}$. Using the same labelling convention as for Operation I, we assume that $G\left[F_{i}\right]$ is drawn in the plane and labelled as in Figure 8(a).

If $f_{i} f_{j} \in E\left(G_{4}(H)\right)$, then $f_{j}$ is obtained from $f_{i}$ by changing the colour of a single vertex in $V(H)$. We label each edge $f_{i} f_{j} \in E\left(G_{4}(H)\right)$ according to the structure of $G\left[F_{i} \cup F_{j}\right]$.

(i) $f_{i}(v) \neq f_{j}(v)$ for $v \in\{a, c\}$. This is analogous to Operation I when the colour is changed on one of $\left\{a_{1}, a_{2}, c_{1}, c_{2}\right\}$, and thus $G\left[F_{i} \cup F_{j}\right]$ is isomorphic to the graph in Figure $8(\mathrm{~b})$. We label the edge $f_{i} f_{j}$ by either a-sq or c-sq as in Operation I.

(ii) $f_{i}(v) \neq f_{j}(v)$ for $v \in\{x, y\}$. This is analogous to Operation II when the colour of $x$ is changed, and thus $G\left[F_{i} \cup F_{j}\right]$ is isomorphic to the graph in Figure 8(d). We label the edge $f_{i} f_{j}$ by e.

(iii) $f_{i}(u) \neq f_{j}(u)$ for vertex $u \in V(H) \backslash\{a, c, x, y\}$. In this case, the vertex labels on $G\left[F_{i}\right]$ and $G\left[F_{j}\right]$ are identical. Thus $\left[F_{i}, F_{j}\right]$ is a perfect matching, and $G\left[F_{i} \cup F_{j}\right]$ is isomorphic to the graph in Figure $8(\mathrm{c})$. We label the edge $f_{i} f_{j}$ by pm.

Remark 5.1. We note that for Operations I-III, if $f_{i} f_{j_{1}}, f_{i} f_{j_{2}} \in E\left(G_{4}(H)\right)$ have the same label that is not e, and $F_{i}^{1}, F_{i}^{2} \subseteq F_{i}$ are incident to the edges of $\left[F_{i}, F_{j_{1}}\right],\left[F_{i}, F_{j_{2}}\right]$, respectively, then $F_{i}^{1}=F_{i}^{2}$. 
Table 3: Summary of Operation III.

\begin{tabular}{c|c|c} 
& Subgraph induced by & \\
Vertex whose colour is changed & $F_{i} \cup F_{j}$ & Label of $f_{i} f_{j}$ \\
\hline \hline$a$ & Figure 8(b) & a-sq \\
\hline$c$ & Figure 8(b) & c-sq \\
\hline$x, y$ & Figure 8(d) & e \\
\hline$u \in V(H) \backslash\{a, c, x, y\}$ & Figure 8(c) & pm
\end{tabular}

\subsection{Operation IV}

We may assume that $f_{i}(x)=1, f_{i}(y)=2, f_{i}(w)=2$ and $f_{i}(z)=3$. Then the pairs of colour available for $\alpha$ and $\beta$, respectively, are

$$
\{(4,3),(3,3),(3,4),(4,4)\},
$$

and the pairs of colours available for $\gamma$ and $\delta$, respectively, are

$$
\{(1,4),(1,1),(4,1),(4,4)\} \text {. }
$$

Thus the subgraph of $G$ induced by $F_{i}$ is isomorphic to $C_{4} \square C_{4}$, and we assume that it is drawn as shown in Figure 9(a), with the rows labelled by the pairs of colours available for $\alpha$ and $\beta$, respectively, and the columns labelled by the pairs of colours available for $\gamma$ and $\delta$, respectively.

If $f_{i} f_{j} \in E\left(G_{4}(H)\right)$, then $f_{j}$ is obtained from $f_{i}$ by changing the colour of a single vertex in $V(H)$; there are three cases to consider.

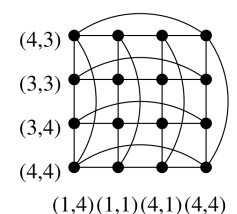

$(1,4)(1,1)(4,1)(4,4)$

(a)

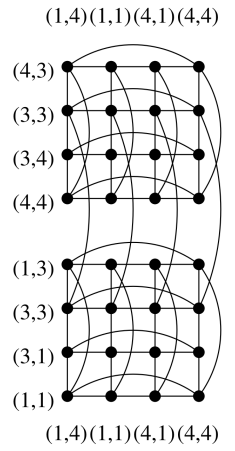

(b)

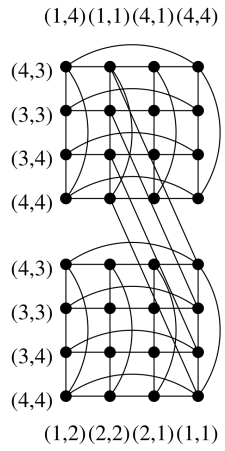

(c)

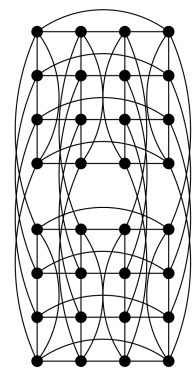

(d)

Figure 9: $G\left[F_{i}\right]$ and $G\left[F_{i} \cup F_{j}\right]$ for Operation IV.

(i) $f_{i}(v) \neq f_{j}(v)$ for $v \in\{x, y\}$. We may assume that $f_{j}(x)=4$. Then the pairs of colours available for $\alpha$ and $\beta$, respectively, are

$$
\{(1,3),(3,3),(3,1),(1,1)\},
$$

and the pairs of colours available for $\gamma$ and $\delta$ are unchanged. Hence, $G\left[F_{i} \cup F_{j}\right]$ is isomorphic to the graph in Figure 9(b). 
(ii) $f_{i}(v) \neq f_{j}(v)$ for $v \in\{w, z\}$. We may assume that $f_{j}(w)=4$. Then the pairs of colours available for $\gamma$ and $\delta$, respectively, are

$$
\{(2,2),(2,1),(1,1),(1,2)\},
$$

and the pairs of colours available for $\alpha$ and $\beta$ are unchanged. Hence, $G\left[F_{i} \cup F_{j}\right]$ is isomorphic to the graph in Figure $9(\mathrm{c})$.

(iii) $f_{i}(u) \neq f_{j}(u)$ for $u \in V(H) \backslash\{x, y, w, z\}$. In this case, the vertex labels on $G\left[F_{i}\right]$ and $G\left[F_{j}\right]$ are identical. Thus $\left[F_{i}, F_{j}\right]$ is a perfect matching, and $G\left[F_{i} \cup F_{j}\right]$ is isomorphic to the graph in Figure $9(\mathrm{~d})$.

Table 4: Summary of Operation IV.

\begin{tabular}{c|c|c} 
& Subgraph induced by & \\
Vertex whose colour is changed & $F_{i} \cup F_{j}$ & Label of $f_{i} f_{j}$ \\
\hline \hline$x, y$ & Figure 9(b) & $\mathrm{r}$ \\
\hline$z, w$ & Figure 9(c) & $\mathrm{c}$ \\
\hline$u \in V(H) \backslash\{x, y, z, w\}$ & Figure 9(d) & $\mathrm{pm}$
\end{tabular}

Remark 5.2. We note that for Operation IV, if $f_{i} f_{j} \in E\left(G_{4}(H)\right)$ has label $\mathrm{r}$ and $e \in$ $\left[F_{i}, F_{j}\right]$, then each colouring corresponding to an end of $e$ assigns the same colour to $\alpha$ and $\beta$. Similarly, if $f_{i} f_{j} \in E\left(G_{4}(H)\right)$ has label $\mathrm{c}$ and $e \in\left[F_{i}, F_{j}\right]$, then each colouring corresponding to an end of $e$ assigns the same colour to $\gamma$ and $\delta$.

\subsection{Operation V}

We may assume that $f_{i}(x)=1, f_{i}(y)=2, f_{i}(w)=2$ and $f_{i}(z)=3$. Then the pairs of colour available for $\alpha$ and $\beta$, respectively, are

$$
\{(3,4),(3,1),(4,1),(4,3)\},
$$

and the pairs of colours available for $\gamma$ and $\delta$, respectively, are

$$
\{(4,1),(1,1),(1,4),(4,4)\} \text {. }
$$

Thus the subgraph of $G_{4}\left(H^{\prime}\right)$ induced by $F_{i}$ is isomorphic to $P_{4} \square C_{4}$, and we assume that it is drawn as shown in Figure 10(a), with the rows labelled by the pairs of colours available for $\alpha$ and $\beta$, respectively, and the columns labelled by the pairs of colours available for $\gamma$ and $\delta$, respectively.

If $f_{i} f_{j} \in E\left(G_{4}(H)\right)$, then $f_{j}$ is obtained from $f_{i}$ by changing the colour of a single vertex in $V(H)$; there are five cases to consider. Since $H$ is a 2-tree, there are vertices $a, b \in V(H)$ such that $H[\{x, y, a\}] \cong K_{3}$ and $H[\{w, z, b\}] \cong K_{3}$. Observe $f_{i}(a) \in\{3,4\}$ and $f_{i}(b) \in\{1,4\}$. We may assume that $f_{i}(a)=4$ and $f_{i}(b)=1$. Even though $b$ (respectively, $a$ ) could be equal to $x$ or $y$ (respectively, $w$ or $z$ ), this does not affect the argument. 


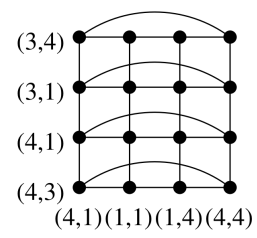

(a)

$(4,1)(1,1)(1,4)(4,4)$

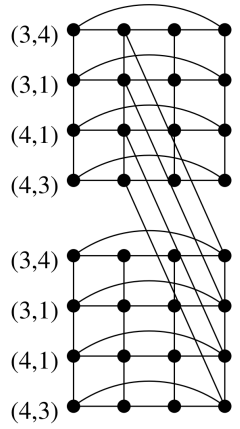

$(3,1)(3,3)(1,3)(1,1)$

(d)
$(4,1)(1,1)(1,4)(4,4)$

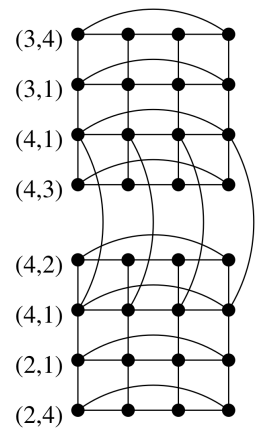

(b)
$(4,1)(1,1)(1,4)(4,4)$

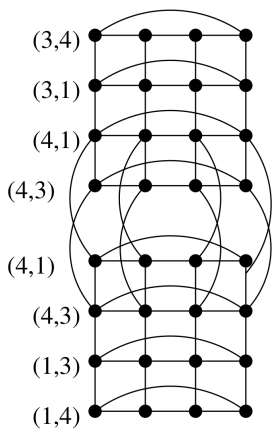

(c)

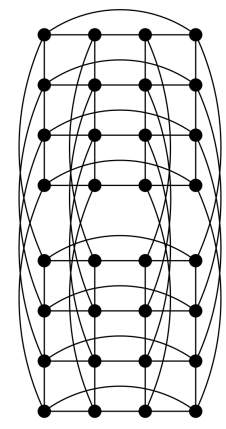

(f)

Figure 10: Subgraphs induced by $F_{i} \cup F_{j}$ for Operations V and IX.

(i) $f_{i}(y) \neq f_{j}(y)$. Then $f_{j}(y)=3$ and the pairs of colours available for $\alpha$ and $\beta$, respectively, are

$$
\{(4,2),(4,1),(2,1),(2,4)\}
$$

and the pairs of colours available for $\gamma$ and $\delta$ are unchanged. Hence, $G\left[F_{i} \cup F_{j}\right]$ is isomorphic to the graph in Figure 10(b) with appropriate labels.

(ii) $f_{i}(x) \neq f_{j}(x)$. Then $f_{j}(x)=3$ and the pairs of colours available for $\alpha$ and $\beta$, respectively, are

$$
\{(4,1),(4,3),(1,3),(1,4)\}
$$

and the pairs of colours available for $\gamma$ and $\delta$ are unchanged. Hence, $G\left[F_{i} \cup F_{j}\right]$ is isomorphic to the graph in Figure 10(c).

(iii) $f_{i}(z) \neq f_{j}(z)$. Then $f_{j}(z)=4$ and the pairs of colours available for $\gamma$ and $\delta$, respectively, are

$$
\{(3,3),(1,3),(1,1),(3,1)\}
$$


and the pairs of colours available for $\alpha$ and $\beta$ are unchanged. Hence, $G\left[F_{i} \cup F_{j}\right]$ is isomorphic to the graph in Figure 10(d).

(iv) $f_{i}(w) \neq f_{j}(w)$. Then $f_{j}(w)=4$ and the pairs of colours available for $\gamma$ and $\delta$, respectively, are

$$
\{(3,3),(1,3),(1,1),(3,1)\},
$$

and the pairs of colours available for $\alpha$ and $\beta$ are unchanged. Hence, $G\left[F_{i} \cup F_{j}\right]$ is isomorphic to the graph in Figure 10(d).

(v) $f_{i}(u) \neq f_{j}(u) u \in V(H) \backslash\{x, y, w, z\}$. In this case, the vertex labels on $G\left[F_{i}\right]$ and $G\left[F_{j}\right]$ are identical. Thus $\left[F_{i}, F_{j}\right]$ is a perfect matching, and $G\left[F_{i} \cup F_{j}\right]$ is isomorphic to the graph in Figure 10(f).

Table 5: Summary of Operation V.

\begin{tabular}{c|c|c} 
& Subgraph induced by & \\
Vertex whose colour is changed & $F_{i} \cup F_{j}$ & Label of $f_{i} f_{j}$ \\
\hline \hline$y$ & Figure 10(b) & $\mathrm{r}$ \\
\hline$x$ & Figure 10(c) & $\mathrm{rr}$ \\
\hline$z, w$ & Figure 10(d) & $\mathrm{c}$ \\
\hline$u \in V(H) \backslash\{x, y, z, w\}$ & Figure 10(f) & $\mathrm{pm}$
\end{tabular}

We informally refer to the rows and columns of vertices in $G\left[F_{i}\right]$ according to the drawing in Figure 10(a). For Operations IV and VI through IX we use a similar convention.

Remark 5.3. We note that for Operation $\mathrm{V}$, if $f_{i} f_{j} \in E\left(G_{4}(H)\right)$ has label $\mathrm{r}$, then the set of vertices $S_{i, j} \subseteq F_{i}$ incident to the edges of $\left[F_{i}, F_{j}\right]$ consists of row two or three ${ }^{2}$. If $f_{i} f_{j} \in E\left(G_{4}(H)\right)$ has label $\mathrm{rr}$, then the set of vertices $S_{i, j} \subseteq F_{i}$ incident to the edges of $\left[F_{i}, F_{j}\right]$ consists of rows one and two, or rows three and four. If $f_{i} f_{j} \in E\left(G_{4}(H)\right)$ has label $\mathrm{c}$ and $e \in\left[F_{i}, F_{j}\right]$, then each colouring corresponding to an end of $e$ assigns the same colour to $\gamma$ and $\delta$.

\subsection{Operation VI}

We may assume that $f_{i}(x)=1, f_{i}(y)=2, f_{i}(z)=3$ and $f_{i}(w)=1$. Then the pairs of colour available for $\alpha$ and $\beta$, respectively, are

$$
\{(3,4),(3,1),(4,1),(4,3)\},
$$

and the pairs of colours available for $\gamma$ and $\delta$, respectively, are

$$
\{(2,4),(2,1),(4,1),(4,2)\} \text {. }
$$

Thus $G\left[F_{i}\right]$ is isomorphic to $P_{4} \square P_{4}$, and we assume that it is drawn in the plane as shown in Figure 11(a), with the rows labelled by the pairs of colours available for $\alpha$ and $\beta$, respectively, and the columns labelled by the pairs of colours available for $\gamma$ and $\delta$, respectively.

\footnotetext{
${ }^{2}$ Rows are numbered from top to bottom and columns from left to right.
} 
$(2,4)(2,1)(4,1)(4,2)$

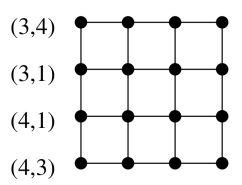

(a)

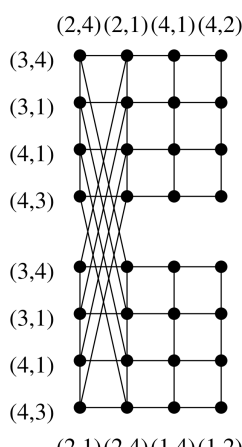

(e)
$(2,4)(2,1)(4,1)(4,2)$

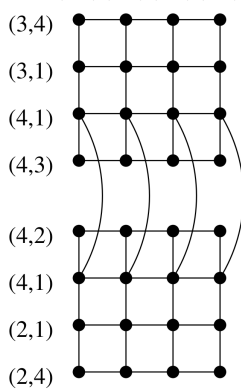

(b)

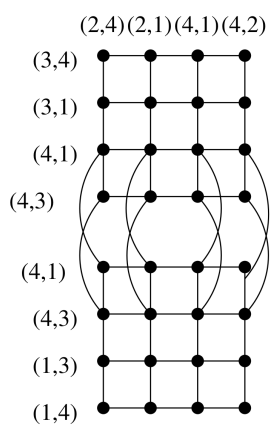

(c)

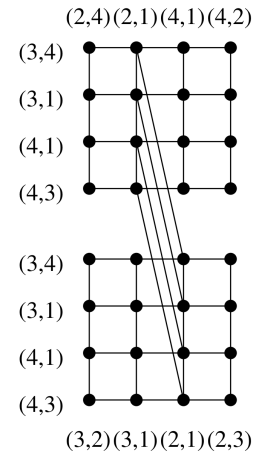

(d)
$(2,4)(2,1)(4,1)(4,2)$

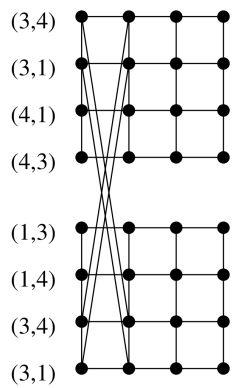

$(2,1)(2,4)(1,4)(1,2)$

(g)

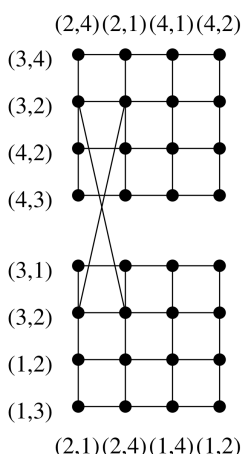

(h)

Figure 11: Subgraphs induced by $F_{i} \cup F_{j}$ for Operations VI, VII and VIII.

If $f_{i} f_{j} \in E\left(G_{4}(H)\right)$, then $f_{j}$ is obtained from $f_{i}$ by changing the colour of a single vertex in $V(H)$; there are five cases to consider. Since $H$ is a 2-tree, there are vertices $a, b \in V(H)$ such that $H[\{x, y, a\}] \cong K_{3}$ and $H[\{w, z, b\}] \cong K_{3}$. Observe $f_{i}(a) \in\{3,4\}$ and $f_{i}(b) \in\{2,4\}$. We may assume that $f_{i}(a)=4$ and $f_{i}(b)=2$. Even though $b$ (respectively, $a$ ) could be equal to $x$ or $y$ (respectively, $w$ or $z$ ), this does not affect the argument.

(i) $f_{i}(y) \neq f_{j}(y)$. Then $f_{j}(y)=3$, and the pairs of colours available for $\alpha$ and $\beta$, respectively, are

$$
\{(4,2),(4,1),(2,1),(2,4)\}
$$

and the pairs of colours available for $\gamma$ and $\delta$ are unchanged. Hence, $G\left[F_{i} \cup F_{j}\right]$ is isomorphic the the graph in Figure 11(b).

(ii) $f_{i}(x) \neq f_{j}(x)$. Then $f_{j}(x)=3$, and the pairs of colours available for $\alpha$ and $\beta$, respectively, are

$$
\{(4,1),(4,3),(1,3),(1,4)\},
$$

while the pairs of colours available for $\gamma$ and $\delta$ are unchanged. Hence, $G\left[F_{i} \cup F_{j}\right]$ is isomorphic to the graph in Figure 11(c). 
(iii) $f_{i}(z) \neq f_{j}(z)$. Then $f_{j}(z)=4$ and the pairs of colours available for $\gamma$ and $\delta$, respectively, are

$$
\{(3,2),(3,1),(2,1),(2,3)\},
$$

while the pairs of colours available for $\alpha$ and $\beta$ are unchanged. Hence, $G\left[F_{i} \cup F_{j}\right]$ is isomorphic the the graph in Figure 11(d).

(iv) $f_{i}(w) \neq f_{j}(w)$. Then $f_{j}(w)=4$ and the pairs of colours available for $\gamma$ and $\delta$, respectively, are

$$
\{(2,1),(2,4),(1,4),(1,2)\},
$$

while the pairs of colours available for $\alpha$ and $\beta$ are unchanged. Hence, $G\left[F_{i} \cup F_{j}\right]$ is isomorphic the the graph in Figure 11(e).

(v) $f_{i}(u) \neq f_{j}(u)$ for some $u \in V(H) \backslash\{x, y, z, w\}$. In this case, the vertex labels on $G\left[F_{i}\right]$ and $G\left[F_{j}\right]$ are identical. Thus $\left[F_{i}, F_{j}\right]$ is a perfect matching, and $G\left[F_{i} \cup F_{j}\right]$ is isomorphic to the graph in Figure 11(f).

Table 6: Summary of Operation VI.

\begin{tabular}{c|c|c} 
& Subgraph induced by & \\
Vertex whose colour is changed & $F_{i} \cup F_{j}$ & Label of $f_{i} f_{j}$ \\
\hline \hline$y$ & Figure 11(b) & $\mathrm{r}$ \\
\hline$x$ & Figure 11(c) & $\mathrm{rr}$ \\
\hline$z$ & Figure 11(d) & $\mathrm{c}$ \\
\hline$w$ & Figure 11(e) & $\mathrm{cc}$ \\
\hline$u \in V(H) \backslash\{x, y, z, w\}$ & Figure 11(f) & $\mathrm{pm}$
\end{tabular}

Remark 5.4. We note that for Operation VI, if $f_{i} f_{j} \in E\left(G_{4}(H)\right)$ has label $\mathrm{r}$ (respectively, c), then the set of vertices $S_{i, j} \subseteq F_{i}$ incident to the edges of $\left[F_{i}, F_{j}\right]$ consists of row (respectively, column) two or three. If $f_{i} f_{j} \in E\left(G_{4}(H)\right)$ has label rr (respectively, cc), then the set of vertices $S_{i, j} \subseteq F_{i}$ incident to the edges of $\left[F_{i}, F_{j}\right]$ consists of rows (respectively, columns) one and two or rows (respectively, columns) three and four.

\subsection{Operation VII}

We may assume that $f_{i}(x)=1, f_{i}(y)=2$ and $f_{i}(z)=3$. Then the pairs of colours available for $\alpha$ and $\beta$, respectively, are

$$
\{(3,4),(3,1),(4,1),(4,3)\},
$$

and the pairs of colours available for $\gamma$ and $\delta$, respectively, are

$$
\{(2,4),(2,1),(4,1),(4,2)\} .
$$

Thus $G\left[F_{i}\right]$ is isomorphic to $P_{4} \square P_{4}$, and we assume that it is drawn in the plane as shown in Figure 11(a) with rows labelled by the pairs of colours available for $\alpha$ and $\beta$, respectively, and the columns labelled by the pairs of colours available for $\gamma$ and $\delta$, respectively.

If $f_{i} f_{j} \in E\left(G_{4}(H)\right)$, then $f_{j}$ is obtained from $f_{i}$ by changing the colour of a single vertex in $V(H)$. There are four cases to consider. 
(i) $f_{i}(x) \neq f_{j}(x)$. We may assume that $f_{j}(x)=4$. Then the pairs of colours available for $\alpha$ and $\beta$, respectively, are

$$
\{(3,1),(3,4),(1,4),(1,3)\}
$$

and the pairs of colours available for $\gamma$ and $\delta$, respectively, are

$$
\{(2,1),(2,4),(1,4),(1,2)\}
$$

Hence, $G\left[F_{i} \cup F_{j}\right]$ is isomorphic to the graph in Figure 11(g).

(ii) $f_{i}(y) \neq f_{j}(y)$. This is analogous to Operation VI when the colour of $y$ is changed, and thus $G\left[F_{i} \cup F_{j}\right]$ is isomorphic to the graph in Figure 11(b).

(iii) $f_{i}(z) \neq f_{j}(z)$. This is analogous to Operation VI when the colour of $z$ is changed, and thus $G\left[F_{i} \cup F_{j}\right]$ is isomorphic to the graph in Figure 11(d).

(iv) $f_{i}(u) \neq f_{j}(u)$ for some $u \in V(H) \backslash\{x, y, z\}$. In this case, the vertex labels on $G\left[F_{i}\right]$ and $G\left[F_{j}\right]$ are identical. Thus $\left[F_{i}, F_{j}\right]$ is a perfect matching, and $G\left[F_{i} \cup F_{j}\right]$ is isomorphic to the graph in Figure 11(f).

Table 7: Summary of Operation VII.

\begin{tabular}{c|c|c} 
& Subgraph induced by & \\
Vertex whose colour is changed & $F_{i} \cup F_{j}$ & Label of $f_{i} f_{j}$ \\
\hline \hline$x$ & Figure 11(g) & sq \\
\hline$y$ & Figure 11(b) & $\mathrm{r}$ \\
\hline$z$ & Figure 11(d) & $\mathrm{c}$ \\
\hline$u \in V(H) \backslash\{x, y, z\}$ & Figure 11(f) & $\mathrm{pm}$
\end{tabular}

Remark 5.5. We note that for Operation VII, if $f_{i} f_{j} \in E\left(G_{4}(H)\right)$ has label r (respectively, c), then the set of vertices $S_{i, j} \subseteq F_{i}$ incident to the edges of $\left[F_{i}, F_{j}\right]$ consists of row (respectively, column) two or three. If $f_{i} f_{j} \in E\left(G_{4}(H)\right)$ has label sq, then the set of vertices $S_{i, j} \subseteq F_{i}$ incident to the edges of $\left[F_{i}, F_{j}\right]$ induces a four-cycle using a degree two vertex of $G\left[F_{i}\right]$.

\subsection{Operation VIII}

We may assume that $f_{i}(x)=1, f_{i}(y)=2$ and $f_{i}(z)=3$. Then the pairs of colours available for $\alpha$ and $\beta$, respectively, are

$$
\{(4,3),(4,2),(3,2),(3,4)\},
$$

and the pairs of colours available for $\gamma$ and $\delta$, respectively, are

$$
\{(2,4),(2,1),(4,1),(4,2)\} \text {. }
$$

Thus $G\left[F_{i}\right]$ is isomorphic to $P_{4} \square P_{4}$, and we assume that it is drawn in the plane as shown in Figure 11(a) with rows labelled by the pairs of colours available for $\alpha$ and $\beta$, respectively, 
and the columns labelled by the pairs of colours available for $\gamma$ and $\delta$, respectively (but not the same labels as in the figure).

If $f_{i} f_{j} \in E\left(G_{4}(H)\right)$, then $f_{j}$ is obtained from $f_{i}$ by changing the colour of a single vertex in $V(H)$, and there are four cases.

(i) $f_{i}(x) \neq f_{j}(x)$. We may assume that $f_{j}(x)=4$. Then the pairs of colours available for $\alpha$ and $\beta$, respectively, are

$$
\{(1,3),(1,2),(3,2),(3,1)\},
$$

and the pairs of colours available for $\gamma$ and $\delta$, respectively, are

$$
\{(2,1),(2,4),(1,4),(1,2)\} \text {. }
$$

Hence, $G\left[F_{i} \cup F_{j}\right]$ is isomorphic to the graph in Figure 11(h) with appropriate labels.

(ii) $f_{i}(y) \neq f_{j}(y)$. This is analogous to Operation VI when the colour of $x$ is changed, and thus $G\left[F_{i} \cup F_{j}\right]$ is isomorphic to the graph in Figure 11(c) with appropriate labels.

(iii) $f_{i}(z) \neq f_{j}(z)$. This is analogous to Operation VI when the colour of $z$ is changed, and thus $G\left[F_{i} \cup F_{j}\right]$ is isomorphic to the graph in Figure 11(d) with appropriate labels.

(iv) $f_{i}(u) \neq f_{j}(u)$ for some $u \in V(H) \backslash\{x, y, z\}$. In this case, the vertex labels on $G\left[F_{i}\right]$ and $G\left[F_{j}\right]$ are identical. Thus $\left[F_{i}, F_{j}\right]$ is a perfect matching, and $G\left[F_{i} \cup F_{j}\right]$ is isomorphic to the graph in Figure 11(f).

Table 8: Summary of Operation VIII.

\begin{tabular}{c|c|c} 
& Subgraph induced by & \\
Vertex whose colour is changed & $F_{i} \cup F_{j}$ & Label of $f_{i} f_{j}$ \\
\hline \hline$x$ & Figure 11(h) & $\mathrm{e}$ \\
\hline$y$ & Figure 11(c) & $\mathrm{rr}$ \\
\hline$z$ & Figure 11(d) & $\mathrm{c}$ \\
\hline$u \in V(H) \backslash\{x, y, z\}$ & Figure 11(f) & $\mathrm{pm}$
\end{tabular}

Remark 5.6. We note that for Operation VIII, if $f_{i} f_{j} \in E\left(G_{4}(H)\right)$ has label c, then the set of vertices $S_{i, j} \subseteq F_{i}$ incident to the edges of $\left[F_{i}, F_{j}\right]$ consists of column two or three. If $f_{i} f_{j} \in E\left(G_{4}(H)\right)$ has label rr, then the set of vertices $S_{i, j} \subseteq F_{i}$ incident to the edges of $\left[F_{i}, F_{j}\right]$ consists of rows one and two or rows three and four. If $f_{i} f_{j} \in E\left(G_{4}(H)\right)$ has label e, then the set of vertices $S_{i, j} \subseteq F_{i}$ incident to the edges of $\left[F_{i}, F_{j}\right]$ induces an edge that is the first or last edge of row two or row three.

\subsection{Operation IX}

We may assume that $f_{i}(x)=1, f_{i}(y)=2$ and $f_{i}(z)=3$. Then the pairs of colours available for $\alpha$ and $\beta$, respectively, are

$$
\{(4,3),(4,1),(3,1),(3,4)\},
$$


and the pairs of colours available for $\gamma$ and $\delta$, respectively, are

$$
\{(2,2),(2,4),(4,4),(4,2)\} \text {. }
$$

Thus $G\left[F_{i}\right]$ is isomorphic to $P_{4} \square C_{4}$, and we assume that it is drawn in the plane as shown in Figure 10(a) with rows labelled by the pairs of colours available for $\alpha$ and $\beta$, respectively, and the columns labelled by the pairs of colours available for $\gamma$ and $\delta$, respectively (but not the same labels as in the figure).

If $f_{i} f_{j} \in E\left(G_{4}(H)\right)$, then $f_{j}$ is obtained from $f_{i}$ by changing the colour of a single vertex in $V(H)$; there are four cases.

(i) $f_{i}(x) \neq f_{j}(x)$. We may assume that $f_{j}(x)=4$. Then the pairs of colours available for $\alpha$ and $\beta$, respectively, are

$$
\{(3,1),(3,4),(1,4),(1,3)\}
$$

and the pairs of colours available for $\gamma$ and $\delta$, respectively, are

$$
\{(2,1),(2,2),(1,2),(1,1)\} .
$$

Hence, $G\left[F_{i} \cup F_{j}\right]$ is isomorphic to the graph in Figure 10(e) with appropriate labels.

(ii) $f_{i}(y) \neq f_{j}(y)$. This is analogous to Operation $\mathrm{V}$ when the colour of $y$ is changed, and thus $G\left[F_{i} \cup F_{j}\right]$ is isomorphic to the graph in Figure 10(b) with appropriate labels.

(iii) $f_{i}(z) \neq f_{j}(z)$. This is analogous to Operation $\mathrm{V}$ when the colour of $z$ is changed, and thus $G\left[F_{i} \cup F_{j}\right]$ is isomorphic to the graph in Figure 10(d) with appropriate labels.

(iv) $f_{i}(u) \neq f_{j}(u)$ for some $u \in V(H) \backslash\{x, y, z\}$. In this case, the vertex labels on $G\left[F_{i}\right]$ and $G\left[F_{j}\right]$ are identical. Thus $\left[F_{i}, F_{j}\right]$ is a perfect matching, and $G\left[F_{i} \cup F_{j}\right]$ is isomorphic to the graph in Figure 10(f).

Table 9: Summary of Operation IX.

\begin{tabular}{c|c|c} 
& Subgraph induced by & \\
Vertex whose colour is changed & $F_{i} \cup F_{j}$ & Label of $f_{i} f_{j}$ \\
\hline \hline$x$ & Figure 10(e) & $\mathrm{e}$ \\
\hline$y$ & Figure 10(b) & $\mathrm{r}$ \\
\hline$z$ & Figure 10(d) & $\mathrm{c}$ \\
\hline$u \in V(H) \backslash\{x, y, z\}$ & Figure 10(f) & $\mathrm{pm}$
\end{tabular}

Remark 5.7. We note that for Operation IX, if $f_{i} f_{j} \in E\left(G_{4}(H)\right)$ has label $\mathrm{r}$ and $e \in$ $\left[F_{i}, F_{j}\right]$, then each colouring corresponding to an end of $e$ assigns the same colour to $\alpha$ and $\beta$. Similarly, if $f_{i} f_{j} \in E\left(G_{4}(H)\right)$ has label $\mathrm{c}$ and $e \in\left[F_{i}, F_{j}\right]$, then each colouring corresponding to an end of $e$ assigns the same colour to $\gamma$ and $\delta$. If $f_{i} f_{j} \in E\left(G_{4}(H)\right)$ has label e and $e \in\left[F_{i}, F_{j}\right]$, then the set of vertices $S_{i, j} \subseteq F_{i}$ incident to the edges of $\left[F_{i}, F_{j}\right]$ induces an edge that is either the first or last edge in a column, and each colouring corresponding to an end of $e$ assigns the same colour to $\gamma$ and $\delta$. 


\section{4-colouring graphs of 2-trees of diameter at least three}

Let $H$ be a 2-tree, and let $H^{\prime}$ be a 2-tree obtained from $H$ by applying one of the Operations I through IX. We prove $G_{4}\left(H^{\prime}\right)$ has a Hamilton cycle.

\subsection{Operations I, II and III}

We first prove a result about Hamilton cycles in a cube $Q_{3}$ that will later be used to show the existence of edges satisfying Lemma 2.7. In this section, we let each face label in Figure 12(a) denote the 4-cycle bounding that face.

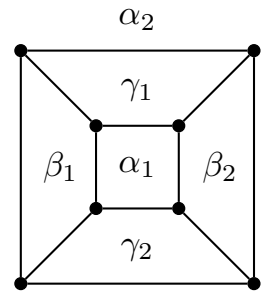

(a)

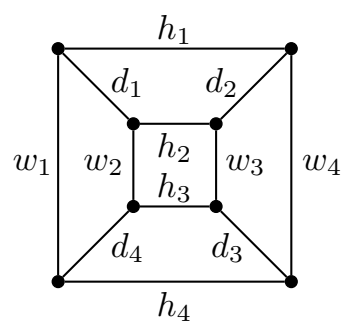

(b)

Figure 12: Labelling $G\left[F_{i}\right]$.

We label the six Hamilton cycles of a plane drawing of $Q_{3}$ as shown in Figure 13.

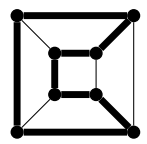

(a) $\sqsubset$

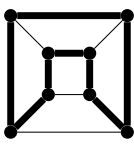

(b) $П$

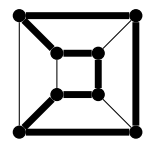

(c) 二

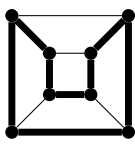

(d) $\sqcup$

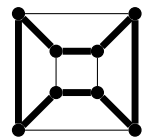

(e) $\mathrm{H}$

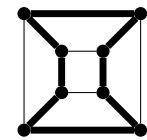

(f) 工

Figure 13: Labels for the Hamilton cycles of $Q_{3}$.

To simplify notation for multisets, we write $n Z$ to mean $n$ copies of $Z$.

Lemma 6.1. Let $Q \cong Q_{3}$ be drawn as in Figure 12(a), and let e be an edge of $Q$. Let $\mathcal{Z}=\left\{Z_{1}, Z_{2}, \ldots, Z_{n}\right\}$ be a multiset such that $\mathcal{Z} \subseteq\left\{2 \alpha_{1}, 2 \beta_{1}, 2 \gamma_{1}, 5 Q_{3}\right\}$ and $n \leq 5$. That is, each $Z_{i}$ is an induced subgraph of $Q$ and is either the entire 3-cube or one of the 4-cycles of $Q$ labelled by $\alpha_{1}, \beta_{1}$, or $\gamma_{1}$. Then there exists a Hamilton cycle in $Q$ containing distinct edges $\left\{e, e_{1}, e_{2}, \ldots, e_{n}\right\}$ such that $e_{i} \in E\left(Z_{i}\right)$, for $1 \leq i \leq n$.

Proof. It is enough to prove the result when $n=5$ and $\mathcal{Z}=\left\{2 \alpha_{1}, 2 \beta_{1}, \gamma_{1}\right\}$. Note that the Hamilton cycles $\sqcup$ and $\sqcap$ in $Q$ each contain three edges of $\alpha_{1}$, three edges of $\beta_{1}$, and two edges of $\gamma_{1}$. When any single edge is deleted from $\sqcup$ or $\sqcap$, we see that the resulting Hamilton path contains five distinct edges, two from $\alpha_{1}$, two from $\beta_{1}$ and one from $\gamma_{1}$. Since at least one of $\sqcup$ and $\sqcap$ contains the edge $e$, either $\sqcup$ or $\sqcap$ contains distinct edges $\left\{e, e_{1}, e_{2}, \ldots, e_{5}\right\}$ such that $e_{i} \in E\left(Z_{i}\right)$, for $1 \leq i \leq 5$. 
Lemma 6.2. Let $Q \cong Q_{3}$ be drawn as in Figure 12(a) with edges labelled as in Figure $12(b)$. Assume $\left\{e, e^{\prime}\right\} \subseteq E(Q)$ with $e \in\left\{w_{1}, w_{2}, w_{3}, w_{4}\right\}$. Let $\mathcal{Z}=\left\{Z_{1}, Z_{2}, \ldots, Z_{n}\right\}$ be a multiset such that $\mathcal{Z} \subseteq\left\{\alpha_{1}, \beta_{1}, 2 \gamma_{1}, 4 Q_{3}\right\}$ and $n \leq 4$. That is, each $Z_{i}$ is an induced subgraph of $Q$ and is either the entire 3-cube or one of the 4-cycles of $Q$ labelled by $\alpha_{1}, \beta_{1}$, or $\gamma_{1}$. Then there exists a Hamilton cycle in $Q$ containing distinct edges $\left\{e, e^{\prime}, e_{1}, e_{2}, \ldots, e_{n}\right\}$ such that $e_{i} \in E\left(Z_{i}\right)$, for $1 \leq i \leq n$.

Proof. It is enough to prove the result when $n=4$ and $\mathcal{Z}=\left\{\alpha_{1}, \beta_{1}, 2 \gamma_{1}\right\}$ with $Z_{1}:=\alpha_{1}$, $Z_{2}:=\beta_{1}, Z_{3}:=\gamma_{1}$ and $Z_{4}:=\gamma_{1}$. Let $H:=\left\{h_{1}, h_{2}, h_{3}, h_{4}\right\}, W:=\left\{w_{1}, w_{2}, w_{3}, w_{4}\right\}$ and $D:=\left\{d_{1}, d_{2}, d_{3}, d_{4}\right\}$. For each $Z_{i}$, we designate a set of candidate edges for $e_{i}$ as follows (see Table 10).

Table 10: Cases in the proof of Lemma 6.2.

\begin{tabular}{c|c|c|c|c|c} 
& Cycle & \multicolumn{5}{|c}{ Edges $e_{i}$ assigned to $Z_{i}$} \\
\cline { 3 - 6 }$e^{\prime}, e$ & in $Q$ & $Z_{1}$ & $Z_{2}$ & $Z_{3}$ & $Z_{4}$ \\
\hline \hline$e^{\prime} \in H, e \in\left\{w_{1}, w_{2}\right\}$ & $\sqsubset$ & $\left\{h_{2}, h_{3}\right\} \backslash\left\{e^{\prime}\right\}$ & $\left\{w_{1}, w_{2}\right\} \backslash\{e\}$ & $d_{2}$ & $\left\{h_{1}, h_{2}\right\} \backslash\left\{e^{\prime}\right\}$ \\
\hline$e^{\prime} \in H, e \in\left\{w_{3}, w_{4}\right\}$ & $\sqsupset$ & $\left\{h_{2}, h_{3}\right\} \backslash\left\{e^{\prime}\right\}$ & $d_{4}$ & $d_{1}$ & $\left\{h_{1}, h_{2}\right\} \backslash\left\{e^{\prime}\right\}$ \\
\hline$\left\{e, e^{\prime}\right\}=\left\{w_{1}, w_{2}\right\}$ & $\sqcap$ & $w_{3}$ & $d_{4}$ & $h_{1}$ & $h_{2}$ \\
\hline$e, e^{\prime} \in W,\left\{e, e^{\prime}\right\} \neq\left\{w_{1}, w_{2}\right\}$ & $\sqcup$ & $h_{3}$ & $\left\{w_{1}, w_{2}\right\} \backslash\left\{e, e^{\prime}\right\}$ & $d_{1}$ & $d_{2}$ \\
\hline$e^{\prime} \in D, e \in\left\{w_{1}, w_{4}\right\}$ & $\mathrm{H}$ & $h_{3}$ & $\left\{d_{1}, d_{4}\right\} \backslash\left\{e^{\prime}\right\}$ & $h_{1}$ & $\left\{d_{1}, d_{2}\right\} \backslash\left\{e^{\prime}\right\}$ \\
\hline$e^{\prime} \in D, e \in\left\{w_{2}, w_{3}\right\}$ & $\mathrm{I}$ & $\left\{w_{2}, w_{3}\right\} \backslash\{e\}$ & $\left\{d_{1}, d_{4}\right\} \backslash\left\{e^{\prime}\right\}$ & $h_{1}$ & $\left\{d_{1}, d_{2}\right\} \backslash\left\{e^{\prime}\right\}$
\end{tabular}

As indicated in Table 10, the first case has $e^{\prime} \in H$ and $e \in\left\{w_{1}, w_{2}\right\}$. We claim that $\sqsubset$ has the required property. We take $e_{3}:=d_{2}$, and as $\left|\left\{w_{1}, w_{2}\right\} \backslash\{e\}\right|=1$, we take $\left\{e_{2}\right\}:=\left\{w_{1}, w_{2}\right\} \backslash\{e\}$. Observe that the sets $\left\{h_{2}, h_{3}\right\} \backslash\left\{e^{\prime}\right\}$ and $\left\{h_{1}, h_{2}\right\} \backslash\left\{e^{\prime}\right\}$ are distinct and non-empty. Thus, we may take $e_{1} \in\left\{h_{2}, h_{3}\right\} \backslash\left\{e^{\prime}\right\}$ and $e_{4} \in\left\{h_{1}, h_{2}\right\} \backslash\left\{e^{\prime}\right\}$ so that $e_{1} \neq e_{4}$. The remaining five cases follow using analogous arguments.

Lemma 6.3. Suppose $H^{\prime}$ is obtained from a 2-tree $H$ by applying one of Operations I, II or III. Then $G_{4}\left(H^{\prime}\right)$ has a Hamilton cycle.

Proof. Case 1. Suppose $H^{\prime}$ is obtained from $H$ by applying Operation I. By Lemma 2.5, $G_{4}(H)$ has a spanning tree $T$ with $\Delta(T) \leq 4$. Let $V(T):=\left\{f_{0}, f_{1}, \ldots, f_{N-1}\right\}$ such that $f_{0}$ is a leaf, and root $T$ at $f_{0}$, turning $T$ into a branching, $\vec{T}$, by directing all arcs away from $f_{0}$.

Let $G:=G_{4}\left(H^{\prime}\right)$, and let $F_{i}$ be the set of 4-colourings of $H^{\prime}$ that agree with $f_{i}$ on $V\left(G_{4}(H)\right), 0 \leq i \leq N-1$. Label each $\overrightarrow{f_{i} f_{j}} \in A(\vec{T})$ with the label of $f_{i} f_{j} \in E\left(G_{4}(H)\right)$, as described in Section 5, and let $S_{i, j} \subseteq F_{i}$ denote the vertices incident to the edges of $\left[F_{i}, F_{j}\right]$.

For each arc $\overrightarrow{f_{i} f_{j}} \in A(\vec{T})$, we choose edges $e_{i, j}$ in $G\left[F_{i}\right]$ and $e_{j, i}$ in $G\left[F_{j}\right]$ satisfying conditions (i) and (ii) of Lemma 2.7 as follows. Suppose $\overrightarrow{f_{0} f_{1}} \in A(\vec{T})$. The fact that $G\left[F_{0} \cup F_{1}\right]$ is isomorphic to the graph in Figure 8(b) or 8(c) gives us the flexibility to choose $e_{0,1} \in E\left(G\left[F_{0}\right]\right)$ and $e_{1,0} \in E\left(G\left[F_{1}\right]\right)$ satisfying (ii) of Lemma 2.7.

Edge choosing procedure. Now suppose for some $i, e_{i, k}$ has been chosen in $G\left[F_{i}\right]$ but $e_{i, j}$ has not yet been chosen for each $j$ where $\overrightarrow{f_{i} f_{j}} \in A(\vec{T})$. For this $i$, let $J:=\{j \mid$ 
$\left.\overrightarrow{f_{i} f_{j}} \in A(\vec{T})\right\}$. We choose edges $e_{i, j}$ and $e_{j, i}$ for $j \in J$ as follows. Assume that $G\left[F_{i}\right]$ is drawn as in Figure 12(a). By Remark 5.1, $S_{i, j_{1}}=S_{i, j_{2}}$ whenever $\overrightarrow{f_{i} f_{j_{1}}}$ and $\overrightarrow{f_{i} f_{j_{2}}}$ have the same label. Without loss of generality, we may assume that an arc $\overrightarrow{f_{i} f_{j}}$ with label a-sq has $G\left[S_{i, j}\right]$ isomorphic to the 4-cycle $\alpha_{1}$, an arc $\overrightarrow{f_{i} f_{j}}$ with label b-sq has $G\left[S_{i, j}\right]$ isomorphic to the 4 -cycle $\beta_{1}$, and an arc $\overrightarrow{f_{i} f_{j}}$ with label c-sq has $G\left[S_{i, j}\right]$ isomorphic to the 4-cycle $\gamma_{1}$. For each $j \in J$, let $Z_{j}:=G\left[S_{i, j}\right]$, and define the multiset $\mathcal{Z}:=\left\{Z_{j} \mid j \in J\right\}$. Then each $Z_{j}$ is either a 3 -cube or one of the 4-cycles $\alpha_{1}, \beta_{1}$, or $\gamma_{1}$. Since $f_{i}$ is incident to at most two edges with label a-sq, at most two edges with label b-sq, and at most two edges with label c-sq, $\mathcal{Z} \subseteq\left\{2 \alpha_{1}, 2 \beta_{1}, 2 \gamma_{1}, 5 Q_{3}\right\}$. Observe $|\mathcal{Z}| \leq 4$ since $\Delta(T) \leq 4$. By Lemma 6.1, using $e:=e_{i, k}$, there is a Hamilton cycle $C_{i}$ in $G\left[F_{i}\right]$ and an edge $e_{i, j} \in E\left(Z_{j}\right)$ for each $Z_{j} \in \mathcal{Z}$ such that $e_{i, j_{1}} \neq e_{i, j_{2}}$ whenever $j_{1} \neq j_{2}$. Thus (i) of Lemma 2.7 is satisfied. Furthermore, for each $j \in J$ there is an edge $e_{j, i} \in E\left(G\left[F_{j}\right]\right)$ such that $e_{i, j}$ and $e_{j, i}$ satisfy (ii) of Lemma 2.7.

Now suppose for every $\overrightarrow{f_{i} f_{j}} \in A(\vec{T}), e_{i, j}$ and $e_{j, i}$ have been chosen as above. By construction, (i) and (ii) of Lemma 2.7 are satisfied, and for each $G\left[F_{i}\right], 0 \leq i \leq N-1$, the Hamilton cycle $C_{i}$ satisfies condition (iii) of Lemma 2.7. Therefore, $G$ has a Hamilton cycle.

Case 2. Suppose $H^{\prime}$ is obtained from $H$ by applying Operation II. Let $\mathcal{H}:=G_{4}(H)$ and $V(H):=\left\{f_{0}, f_{1}, \ldots, f_{N-1}\right\}$. For each $1 \leq i \leq 4$ let $V_{i}:=\{c \in V(\mathcal{H}) \mid c(x)=i\}$. Then $\left\{V_{1}, V_{2}, V_{3}, V_{4}\right\}$ is a partition of $V(\mathcal{H})$. Let $L_{i}$ be an assignment of lists with $L_{i}(x):=\{i\}$ and $L_{i}(w):=\{1,2,3,4\}$ for $w \in V(H) \backslash\{x\}$. Note that $G_{L_{i}}(H) \cong \mathcal{H}\left[V_{i}\right]$ and that $\mathcal{H}\left[V_{1}\right] \cong \mathcal{H}\left[V_{2}\right] \cong \mathcal{H}\left[V_{3}\right] \cong \mathcal{H}\left[V_{4}\right]$. Thus, $\mathcal{H}$ can be partitioned into four copies isomorphic to $G_{L_{1}}(H)$ with edges between pairs of copies. Furthermore, each edge in $E\left(\mathcal{H}\left[V_{i}\right]\right)$, $1 \leq i \leq 4$, has label a-sq, b-sq, c-sq or pm, and each edge with one endpoint in $V_{i}$ and the other endpoint in $V_{j}, i \neq j$, has label e.

By Lemma 2.5, $\mathcal{H}\left[V_{i}\right], 1 \leq i \leq 4$, has a spanning tree $T_{i}$ with $\Delta\left(T_{i}\right) \leq 4$. Note that $\left[V_{i}, V_{j}\right] \neq \emptyset$ for $1 \leq i \neq j \leq 4$. Choose one edge from each of $\left[V_{1}, V_{2}\right],\left[V_{2}, V_{3}\right]$, and $\left[V_{3}, V_{4}\right]$. Without loss of generality, suppose the chosen edges are $f_{1} f_{2} \in\left[V_{1}, V_{2}\right]$, $f_{2}^{\prime} f_{3} \in\left[V_{2}, V_{3}\right]$, and $f_{3}^{\prime} f_{4} \in\left[V_{3}, V_{4}\right]$ such that $f_{i} \in V_{i}, 1 \leq i \leq 4$. Since $f_{1} f_{2}, f_{2}^{\prime} f_{3}$ and $f_{3}^{\prime} f_{4}$ each have label e in $\mathcal{H}$ and each vertex of $V(\mathcal{H})$ is incident to at most one edge with label e, the vertices $f_{1}, f_{2}, f_{2}^{\prime}, f_{3}, f_{3}^{\prime}, f_{4}$ are distinct. Thus, we may assume that $f_{2}^{\prime}=f_{0}$ and $f_{3}^{\prime}=f_{5}$.

Let $T$ be the spanning tree of $\mathcal{H}$ consisting of the union of $T_{1}, T_{2}, T_{3}, T_{4}$, and the edges $\left\{f_{1} f_{2}, f_{0} f_{3}, f_{5} f_{4}\right\}$. Then $\Delta(T) \leq 5$ and the only edges of $T$ with label e are $f_{1} f_{2}, f_{0} f_{3}$ and $f_{4} f_{5}$. Root $T$ at $f_{1}$, turning $T$ into a branching, $\vec{T}$, by directing all arcs away from $f_{1}$. This gives a branching $\overrightarrow{T_{i}}$ for each $T_{i}, 1 \leq i \leq 4$, and by our choice of labels, $f_{i}$ is the root of $\vec{T}_{i}$.

Let $G:=G_{4}\left(H^{\prime}\right)$, and let $F_{i}$ be the set of 4-colourings of $H^{\prime}$ that agree with $f_{i}$ on $V\left(G_{4}(H)\right), 0 \leq i \leq N-1$. Label each $\overrightarrow{f_{i} f_{j}} \in A(\vec{T})$ with the label of $f_{i} f_{j} \in E(\mathcal{H})$, and let $S_{i, j} \subseteq F_{i}$ and $S_{j}^{\prime} \subseteq F_{j}$ denote the vertices incident to the edges of $\left[F_{i}, F_{j}\right]$.

For each arc $\overrightarrow{f_{i} f_{j}} \in A(\vec{T})$, we choose edges $e_{i, j}$ in $G\left[F_{i}\right]$ and $e_{j, i}$ in $G\left[F_{j}\right]$ satisfying conditions (i) and (ii) of Lemma 2.7 as follows. For $(i, j) \in\{(1,2),(0,3),(5,4)\}$ (where $\overrightarrow{f_{i} f_{j}}$ has label e), let $e_{i, j}$ be the unique edge of $G\left[S_{i, j}\right] \subseteq G\left[F_{i}\right]$ and $e_{j, i}$ be the unique edge of $G\left[S_{j}^{\prime}\right] \subseteq G\left[F_{j}\right]$. For $\vec{T}_{1}$ and $\vec{T}_{4}$ we apply the edge choosing procedure used in Case 1 , 
starting with with $e_{1,2}$ in $G\left[F_{1}\right]$ for $\vec{T}_{1}$, and $e_{4,5}$ in $G\left[F_{4}\right]$ for $\vec{T}_{4}$. The resulting set of edges

$$
\left\{e_{i, j}, e_{j, i} \mid \overrightarrow{f_{i} f_{j}} \in\left(A\left(\overrightarrow{T_{1}} \cup \overrightarrow{T_{4}}\right) \cup\left\{\overrightarrow{f_{1} f_{2}}, \overrightarrow{f_{5} f_{4}}\right\}\right)\right\}
$$

and set of cycles $\left\{C_{i} \mid f_{i} \in V_{1} \cup V_{4}\right\}$ satisfy Lemma 2.7. For $\vec{T}_{2}$, we let $f_{M}$ be the parent of $f_{0}$ and $f_{k}$ the parent of $f_{M}$. Let $\vec{T}_{2}^{\prime}$ be the subtree of $\vec{T}_{2}$ obtained by deleting the descendants of $f_{M}$ and $\vec{T}_{2}^{\prime \prime}$ be the subtree of $\vec{T}_{2}$ rooted at $f_{M}$. If $M=2$ then $k=1$, so $e_{M, k}=e_{2,1}$. Otherwise apply the apply the edge choosing procedure used in Case 1 to ${\overrightarrow{T_{2}}}^{\prime}$, starting with $e_{2,1}$ in $G\left[F_{2}\right]$. This leads to the designation of an edge $e_{M, k}$ in $G\left[F_{M}\right]$.

Let $J:=\left\{j \mid \overrightarrow{f_{M} f_{j}} \in A\left(\vec{T}_{2}\right)\right\}$. We choose edges $e_{M, j}$ and $e_{j, M}$ for $j \in J$ as follows. Without loss of generality, we may assume that an arc $\overrightarrow{f_{M} f_{j}}$ with label a-sq has $G\left[S_{M, j}\right]$ isomorphic to the 4-cycle $\alpha_{1}$, an arc $\overrightarrow{f_{M} f_{j}}$ with label b-sq has $G\left[S_{M, j}\right]$ isomorphic to the 4-cycle $\beta_{1}$, an arc $\overrightarrow{f_{M} f_{j}}$ with label c-sq has $G\left[S_{M, j}\right]$ isomorphic to the 4-cycle $\gamma_{1}$, and an arc with label pm has $G\left[S_{M, j}\right]$ isomorphic to $Q_{3}$. Let $\mathcal{Z}$ be a multiset consisting of the graphs $Z_{j}:=G\left[S_{M, j}\right]$ for $j \in J$.

Suppose there exists $\ell \in(J \backslash\{0\})$ such that both $\overrightarrow{f_{M} f_{0}}$ and $f_{M} f_{\ell}$ have label c-sq. We apply the edge choosing procedure used in Case 1 to $\vec{T}_{2}^{\prime \prime}$, with chosen edge $e_{M, k}$. The resulting set of edges $\left\{e_{M, j} \mid j \in J\right\}$ and cycle $C_{M}$ satisfy Lemma 2.7. Observe that $e_{M, 0}, e_{M, \ell} \in E\left(\gamma_{1}\right)$. Suppose $e_{M, 0}=a b$, and let the vertices of $\left[\{a, b\}, F_{0}\right]$ incident to $F_{0}$ be $\{c, d\}$. If $e_{0,3}=c d$ then exchange $e_{M, 0}$ with $e_{M, \ell}$. It now follows that for each $j \in J$ there is an edge $e_{j, M} \in E\left(G\left[F_{j}\right]\right)$ such that $e_{M, j}$ and $e_{j, M}$ satisfy (ii) of Lemma 2.7, with $e_{0, M} \neq e_{0,3}$.

Otherwise $\overrightarrow{f_{M} f_{0}}$ is the only arc in $\left\{\overrightarrow{f_{M} f_{j}} \mid j \in J\right\}$ labelled c-sq, or $\overrightarrow{f_{M} f_{0}}$ has label a-sq, b-sq, or pm. To ensure (i) of Lemma 2.7 is satisfied for $i=0$, we duplicate $Z_{0} \in \mathcal{Z}$ and apply Lemma 6.1. Let $Z_{0^{\prime}}:=Z_{0}$ and $F_{0^{\prime}}:=F_{0}$. Observe $\left|\mathcal{Z} \cup\left\{Z_{0^{\prime}}\right\}\right| \leq 5$ since $\Delta(T) \leq 4$. Since $f_{M}$ is incident to at most one edge with label a-sq, at most one edge with label b-sq, and at most two edges with label c-sq, $\left(\mathcal{Z} \cup\left\{Z_{0^{\prime}}\right\}\right) \subseteq\left\{2 \alpha_{1}, 2 \beta_{1}, 2 \gamma_{1}, 5 Q_{3}\right\}$. By Lemma 6.1 applied to $\mathcal{Z} \cup\left\{Z_{0^{\prime}}\right\}$, and using $e:=e_{M, k}$, there is a Hamilton cycle $C_{M}$ in $G\left[F_{M}\right]$ and edges $e_{M, j} \in E\left(Z_{j}\right)$ for each $j \in\left(J \cup\left\{0^{\prime}\right\}\right)$ such that $e_{M, j_{1}} \neq e_{M, j_{2}}$ whenever $j_{1} \neq j_{2}$. Thus (i) of Lemma 2.7 is satisfied for $i=M$. Furthermore, for each $j \in\left(J \cup\left\{0^{\prime}\right\}\right)$ there is an edge $e_{j, M} \in E\left(G\left[F_{j}\right]\right)$ such that $e_{j, M}$ and $e_{M, j}$ satisfy (ii) of Lemma 2.7. Since $e_{M, 0^{\prime}} \neq e_{M, 0}$, we have $e_{0, M} \neq e_{0^{\prime}, M}$, and hence, one of $e_{0, M}$ and $e_{0^{\prime}, M}$ is different from $e_{0,3}$. If $e_{0, M} \neq e_{0,3}$ then we ignore $e_{0^{\prime}, M}$ and $e_{M, 0^{\prime}}$; otherwise, redefine $e_{0, M}$ to be $e_{0^{\prime}, M}$ and $e_{M, 0}$ to be $e_{M, 0^{\prime}}$ so that (i) of Lemma 2.7 is satisfied for $i=0$.

Finally, let $G\left[F_{0}\right] \cong Q_{3}$ be drawn as in Figure 12(a) with edges labelled as in Figure 12(b). Observe that $e=e_{0,3} \in\left\{w_{1}, w_{2}, w_{3}, w_{4}\right\}$ and let $e^{\prime}:=e_{0, M}$. Let $J:=\{j \mid$ $\left.\overrightarrow{f_{0} f_{j}} \in A\left(\overrightarrow{T_{2}}\right)\right\}$. We choose edges $e_{0, j}$ and $e_{j, 0}$ for $j \in J$ as follows. Without loss of generality, we may assume that an arc $\overrightarrow{f_{0} f_{j}}$ with label a-sq has $G\left[S_{0, j}\right]$ isomorphic to the 4-cycle $\alpha_{1}$, an arc $\overrightarrow{f_{0} f_{j}}$ with label b-sq has $G\left[S_{0, j}\right]$ isomorphic to the 4-cycle $\beta_{1}$, an arc $\overrightarrow{f_{0} f_{j}}$ with label c-sq has $G\left[S_{0, j}\right]$ isomorphic to the 4-cycle $\gamma_{1}$, and an arc with label pm has $G\left[S_{0, j}\right]$ isomorphic to $Q_{3}$. Let $\mathcal{Z}$ be a multiset consisting of the graphs $Z_{j}:=G\left[S_{0, j}\right]$ for $j \in J$. Observe $\mathcal{Z} \subseteq\left\{\alpha_{1}, \beta_{1}, 2 \gamma_{1}, 4 Q_{3}\right\}$ and $|\mathcal{Z}| \leq 4$. By Lemma 6.2, there exists a Hamilton cycle $C_{0}$ in $G\left[F_{0}\right]$ containing distinct edges $\left\{e, e^{\prime}\right\} \cup\left\{e_{0, j} \mid j \in J\right\}$ such that 
$e_{0, j} \in E\left(Z_{j}\right)$ for $j \in J$. Thus (i) of Lemma 2.7 is satisfied for $i=0$. Furthermore, for each $j \in J$ there is an edge $e_{j, 0} \in E\left(G\left[F_{j}\right]\right)$ so that $e_{j, 0}$ and $e_{0, j}$ satisfy (ii) of Lemma 2.7.

We now apply the edge choosing procedure used in Case 1 to the remaining nodes of $\overrightarrow{T_{2}}$. This designates edges $e_{i, j}$ in $G\left[F_{i}\right]$ and $e_{j, i}$ in $G\left[F_{j}\right]$, and cycles $C_{i}$ satisfying Lemma 2.7. For $\overrightarrow{T_{3}}$ we apply the argument for $\vec{T}_{2}$ giving us edges $e_{i, j}$ in $G\left[F_{i}\right]$ and $e_{j, i}$ in $G\left[F_{j}\right]$, and cycles $C_{i}$ satisfying Lemma 2.7 , for each $\overrightarrow{f_{i} f_{j}} \in A\left(\overrightarrow{T_{3}}\right)$.

Now $e_{i, j}$ and $e_{j, i}$ have been chosen for all $\overrightarrow{f_{i} f_{j}} \in A(\vec{T})$. By construction, each such $e_{i, j}$ and $e_{j, i}$ satisfy (ii) of Lemma 2.7, and for each $G\left[F_{i}\right], 0 \leq i \leq N-1$, the Hamilton cycle $C_{i}$ satisfies condition (iii) of Lemma 2.7. Furthermore, the collection of chosen edges are all distinct. Therefore, $G$ has a Hamilton cycle.

Case 3. Suppose $H^{\prime}$ is obtained from $H$ by applying Operation III. Let $\mathcal{H}:=G_{4}(H)$ and $V(H):=\left\{f_{0}, f_{1}, \ldots, f_{N-1}\right\}$. For each $1 \leq i \neq j \leq 4$ let

$$
V_{i j}:=\{c \in V(\mathcal{H}) \mid c(x)=i \text { and } c(y)=j\} .
$$

Then

$$
V:=\left\{V_{12}, V_{13}, V_{14}, V_{21}, V_{23}, V_{24}, V_{31}, V_{32}, V_{34}, V_{41}, V_{42}, V_{43}\right\}
$$

is a partition of $V(\mathcal{H})$. Note that $\left[V_{\alpha \beta}, V_{\gamma \delta}\right] \neq \emptyset$ if and only if $\alpha=\gamma$ or $\beta=\delta$. Furthermore, each edge in $E\left(\mathcal{H}\left[V_{i j}\right]\right), 1 \leq i \neq j \leq 4$, has label a-sq, c-sq or pm, and each edge with one endpoint in $V_{i_{1} j_{1}}$ and the other endpoint in $V_{i_{2} j_{2}},\left(i_{1}, j_{1}\right) \neq\left(i_{2}, j_{2}\right)$, has label e.

Let $\{i, j, k\} \subset\{1,2,3,4\}$. As $H$ is a 2-tree, $H$ is 3 -colourable and for each $1 \leq$ $i \neq j \leq 4$, there is a unique vertex $c_{i j k} \in V(\mathcal{H})$ with $c_{i j k}(x)=i, c_{i j k}(y)=j$ and $c_{i j k}(w) \in\{i, j, k\}$ for $w \in V(H) \backslash\{x, y\}$.

Consider the ordering

$$
\left(V_{14}, V_{12}, V_{32}, V_{34}, V_{31}, V_{21}, V_{24}, V_{23}, V_{13}, V_{43}, V_{42}, V_{41}\right)
$$

of $V$. For each $V_{i j} \in V \backslash\left\{V_{41}\right\}$, suppose $V_{\ell m}$ immediately follows $V_{i j}$ in the list. Then $|\{i, j\} \cup\{\ell, m\}|=3$, and hence there is a unique $k_{i j} \in\{1,2,3,4\} \backslash(\{i, j\} \cup\{\ell, m\})$ such that $c_{i j k_{i j}} \in V_{i j}$. The ordering of $V$ ensures that for each $V_{i j} \in V \backslash\left\{V_{42}, V_{41}\right\}$ with $V_{\ell m}$ immediately following $V_{i j}$ in the list, $k_{i j} \neq k_{\ell m}$. Choose the edge $c_{14} c_{14}^{\prime}$ from $\left[V_{14}, V_{12}\right]$ with endpoint $c_{14}:=c_{143} \in V_{14}$; note that $c_{14}^{\prime} \neq c_{124}$. For each $\left[V_{i j}, V_{\ell m}\right]$ where $V_{\ell m}$ immediately follows $V_{i j}$ in the list, there is a unique edge $c_{i j} c_{i j}^{\prime}$ with endpoint $c_{i j}=c_{i j k_{i j}} \in V_{i j}$.

By Lemma $2.5, \mathcal{H}\left[V_{i j}\right], 1 \leq i \neq j \leq 4$, has a spanning tree $T_{i j}$ with $\Delta\left(T_{i j}\right) \leq 4$. Let $T$ be the spanning tree of $\mathcal{H}$ with $T_{i j} \subset T, 1 \leq i \neq j \leq 4$, and $c_{i j} c_{i j}^{\prime} \in E(T)$, $1 \leq i \neq j \leq 4$ with $(i, j) \neq(4,1)$. Then $\Delta(T) \leq 5$ and the only edges of $T$ with label e are $c_{i j} c_{i j}^{\prime}, 1 \leq i \neq j \leq 4$ with $(i, j) \neq(4,1)$. Root $T$ at $c_{143} \in V_{14}$, turning $T$ into a branching, $\vec{T}$, by directing all arcs away from $c_{14}$. This gives a branching $\overrightarrow{T_{i j}}$ for each $T_{i j}$, $1 \leq i \neq j \leq 4$. Now repeat the argument in Case 2 for each $\overrightarrow{T_{i j}}, 1 \leq i \neq j \leq 4$.

\subsection{Operations IV to IX}

We introduce some labelled Hamilton cycles of $C_{4} \square C_{4}, P_{4} \square C_{4}$ and $P_{4} \square P_{4}$ to be used in Lemma 2.7 to show the existence of a Hamilton cycle in $G_{4}\left(H^{\prime}\right)$, where $H^{\prime}$ is obtained from a 2 -tree $H$ by applying one of Operations IV through IX. 
Let $\square$ be the edge labelled Hamilton cycle in $C_{4} \square C_{4}$ shown in Figure 14, with the edges in rows two and four labelled by $\mathrm{r}$, the edges in columns two and four labelled by c, and the remaining edges left unlabelled. Let $\sqsubset$ and $\sqsubset^{\prime}$ be the edge labelled Hamilton cycles in $P_{4} \square C_{4}$ shown in Figure 14, and let $I, I^{\prime}$ and $I^{\prime \prime}$ be the edge labelled Hamilton cycles in $P_{4} \square P_{4}$ shown in Figure 14 .

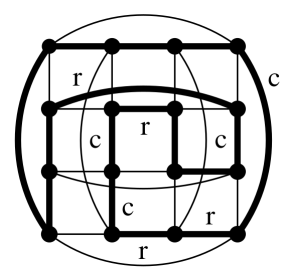

(a) $\mathrm{D}$

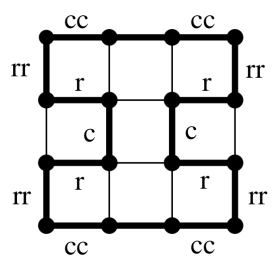

(d) I

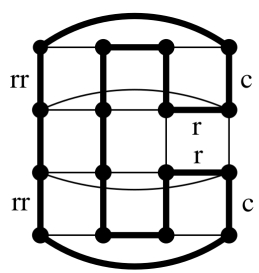

(b) $\sqsubset$

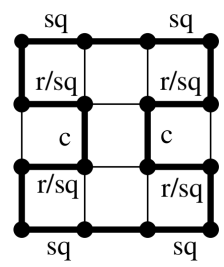

(e) $I^{\prime}$

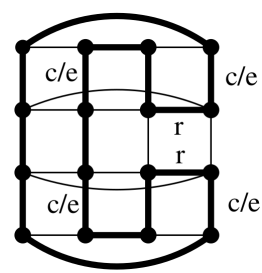

(c) $\leftarrow^{\prime}$

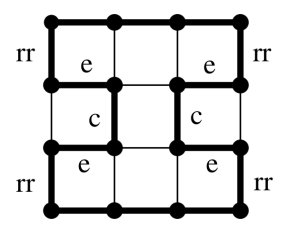

(f) $\perp^{\prime \prime}$

Figure 14: Labelled Hamilton cycles of $C_{4} \square C_{4}, P_{4} \square C_{4}$ and $P_{4} \square P_{4}$.

Lemma 6.4. Suppose $H^{\prime}$ is obtained from a 2-tree $H$ by applying one of Operations IV through IX. Then $G_{4}\left(H^{\prime}\right)$ has a Hamilton cycle.

Proof. By Lemma 2.5, $\mathcal{H}:=G_{4}(H)$ has a spanning tree $T$ with $\Delta(T) \leq 4$. Let $V(T):=$ $\left\{f_{0}, f_{1}, \ldots, f_{N-1}\right\}$ such that $f_{0}$ is a leaf, and root $T$ at $f_{0}$, turning $T$ into a branching, $\vec{T}$, by directing all arcs away from $f_{0}$. Let $G:=G_{4}\left(H^{\prime}\right)$, and let $F_{i}$ be the set of 4-colourings of $H^{\prime}$ that agree with $f_{i}$ on $V(\mathcal{H}), 0 \leq i \leq N-1$. Label each $\overrightarrow{f_{i} f_{j}} \in A(\vec{T})$ with the label of $f_{i} f_{j} \in E(\mathcal{H})$, and let $S_{i, j} \subseteq F_{i}$ and $S_{j}^{\prime} \subseteq F_{j}$ denote the vertices incident to the edges of $\left[F_{i}, F_{j}\right]$.

We first traverse $\vec{T}$ using breadth-first search starting at $f_{0}$ to construct a drawing of each $G\left[F_{i}\right]$ as shown in Figure 9(a) for Operation IV, Figure 10(a) for Operations V and IX, and Figure 11(a) for Operations VI, VII and VIII, so that each drawing has the following property, denoted $(*)$.

(*) The rows are labelled by the pairs of colours available for $\alpha$ and $\beta$, respectively, and the columns are labelled by the pairs of colours available for $\gamma$ and $\delta$, respectively. In the case of Operations IV, V and IX, we further assume that the second and fourth columns have $\gamma$ and $\delta$ the same colour. Also, in the case of Operation IV, we assume the second and fourth rows have $\alpha$ and $\beta$ the same colour.

Start with a drawing of $G\left[F_{0}\right]$ satisfying $(*)$. Assume $\overrightarrow{f_{i} f_{j}} \in A(\vec{T})$ where $G\left[F_{i}\right]$ has been drawn but $G\left[F_{j}\right]$ has not. We draw $G\left[F_{j}\right]$ satisfying $(*)$ as follows. If $\overrightarrow{f_{i} f_{j}}$ has label 
- pm, then draw $G\left[F_{j}\right]$ so that the labels of the rows and columns are in the same order as in $G\left[F_{i}\right]$.

- $\mathbf{r}$, then draw $G\left[F_{j}\right]$ so that the column labels of $G\left[F_{j}\right]$ are in the same order as the column labels of $G\left[F_{i}\right]$, and so that the row number of $S_{j}^{\prime}$ in $G\left[F_{j}\right]$ is the same as that of $S_{i, j}$ in $G\left[F_{i}\right]$. This can be done for Operation IV due to the cyclic structure of $C_{4} \square C_{4}$; furthermore, (*) guarantees that $S_{i, j}$ and $S_{j}^{\prime}$ have row number two or four. For Operations V, VI, VII and IX, this can be done by Remarks 5.3, 5.4, 5.5 and 5.7. Thus, one of the two possible labellings for the rows of $G\left[F_{j}\right]$ gives a drawing of $G\left[F_{j}\right]$ such that the row number of $S_{j}^{\prime}$ in $G\left[F_{j}\right]$ is the same as that of $S_{i, j}$ in $G\left[F_{i}\right]$.

- c , then draw $G\left[F_{j}\right]$ so that the row labels of $G\left[F_{j}\right]$ are in the same order as the row labels of $G\left[F_{i}\right]$, and so that the column number of $S_{j}^{\prime}$ in $G\left[F_{j}\right]$ is the same as that of $S_{i, j}$ in $G\left[F_{i}\right]$. This can always be done using a similar argument as in the case for $\overrightarrow{f_{i} f_{j}}$ having label r. For Operations IV, V and IX, (*) guarantees that $S_{i, j}$ and $S_{j}^{\prime}$ have column number two or four. For Operations VI, VII and VIII, by Remarks 5.4, 5.5 and 5.6, $S_{i, j}$ and $S_{j}^{\prime}$ have column number two or three.

- rr, then draw $G\left[F_{j}\right]$ so that the column labels of $G\left[F_{j}\right]$ are in the same order as the column labels of $G\left[F_{i}\right]$, and so that the set of row numbers of $S_{j}^{\prime}$ in $G\left[F_{j}\right]$ is the same as that of $S_{i, j}$ in $G\left[F_{i}\right]$. This can be done for Operations V, VI and VIII, since by Remarks 5.3, 5.4 and 5.6, the set of row numbers of $S_{i, j}$ and $S_{j}^{\prime}$ is either $\{1,2\}$ or $\{3,4\}$.

- cc, then draw $G\left[F_{j}\right]$ so that the row labels of $G\left[F_{j}\right]$ are in the same order as the row labels of $G\left[F_{i}\right]$, and so that the set of column numbers of $S_{j}^{\prime}$ in $G\left[F_{j}\right]$ is the same as that of $S_{i, j}$ in $G\left[F_{i}\right]$. This can be done for Operation VI, since by Remark 5.4, the set of column numbers of $S_{i, j}$ and $S_{j}^{\prime}$ is either $\{1,2\}$ or $\{3,4\}$.

- sq, then draw $G\left[F_{j}\right]$ satisfying $(*)$.

- e, then draw $G\left[F_{j}\right]$ satisfying $(*)$. Note that for Operation IX, $(*)$ guarantees that $S_{i, j}$ and $S_{j}^{\prime}$ belong to column number two or four.

For Operation IV (respectively, V, VI, VII, VIII, and IX), for each $i, 0 \leq i \leq N-1$, let $C_{i}$ be the Hamilton cycle $\square$ (respectively, $\sqsubset, I, I^{\prime}, エ^{\prime \prime}$, and $\sqsubset^{\prime}$ ) in $G\left[F_{i}\right]$.

We describe how to construct a set of edges

$$
\mathcal{E}:=\left\{e_{i, j}, e_{j, i} \mid f_{i} f_{j} \in E(T)\right\}
$$

so that for each arc $\overrightarrow{f_{i} f_{j}} \in A(\vec{T})$, the edges $e_{i, j}$ in $G\left[F_{i}\right]$ and $e_{j, i}$ in $G\left[F_{j}\right]$ satisfy conditions (i) and (ii) of Lemma 2.7, and so that $e_{i, j} \in E\left(C_{i}\right)$ and $e_{j, i} \in E\left(C_{j}\right)$. Start with $\mathcal{E}:=\emptyset$. We consider the arcs of $\vec{T}$ in the following order according to their labels.

(1) For each $\overrightarrow{f_{i} f_{j}} \in A(\vec{T})$ with label e (Operations VIII and IX), $e_{i, j} \in G\left[F_{i}\right]$ and $e_{j, i} \in G\left[F_{j}\right]$ satisfying condition (ii) of Lemma 2.7 are uniquely determined. Note that for Operation VIII, $e_{i, j} \in C_{i}$ and has label e, and $e_{j, i} \in C_{j}$ and has label e; this follows by the symmetry of $工^{\prime \prime}$ and the drawings $G\left[F_{i}\right]$ and $G\left[F_{j}\right]$. For Operation IX, $e_{i, j} \in C_{i}$ and has label c/e, and $e_{j, i} \in C_{j}$ and has label c/e; this follows from the drawings of $G\left[F_{i}\right]$ and $G\left[F_{j}\right]$, and Remark 5.7. Add $e_{i, j}$ and $e_{j, i}$ to $\mathcal{E}$. 
(2) For each $\overrightarrow{f_{i} f_{j}} \in A(\vec{T})$ with label sq (Operation VII), choose $e_{i, j}$ in $C_{i}$ with label sq and $e_{j, i}$ in $G\left[F_{j}\right]$ satisfying condition (ii) of Lemma 2.7. Note that $e_{j, i} \in C_{j}$ and has label sq or label r/sq; this follows from the drawings of $G\left[F_{i}\right]$ and $G\left[F_{j}\right]$, and Remark 5.7. Add $e_{i, j}$ and $e_{j, i}$ to $\mathcal{E}$.

(3) For each $\overrightarrow{f_{i} f_{j}} \in A(\vec{T})$ with label rr (Operations V, VI and VIII), choose $e_{i, j}$ in $C_{i}$ with label rr and $e_{j, i}$ in $G\left[F_{j}\right]$ satisfying condition (ii) of Lemma 2.7. Note that $e_{j, i} \in C_{j}$ and has label rr; this follows from the drawings of $G\left[F_{i}\right]$ and $G\left[F_{j}\right]$. Add $e_{i, j}$ and $e_{j, i}$ to $\mathcal{E}$.

(4) For each $\overrightarrow{f_{i} f_{j}} \in A(\vec{T})$ with label cc (Operation VI), choose $e_{i, j}$ in $C_{i}$ with label cc and $e_{j, i}$ in $G\left[F_{j}\right]$ satisfying condition (ii) of Lemma 2.7. Note that $e_{j, i} \in C_{j}$ and has label cc; this follows from the drawings of $G\left[F_{i}\right]$ and $G\left[F_{j}\right]$. Add $e_{i, j}$ and $e_{j, i}$ to $\mathcal{E}$.

(5) For each $\overrightarrow{f_{i} f_{j}} \in A(\vec{T})$ with label r (Operations V, VI and IX), choose $e_{i, j}$ in $C_{i}$ with label $\mathrm{r}$ and $e_{j, i}$ in $G\left[F_{j}\right]$ satisfying condition (ii) of Lemma 2.7. Note that $e_{j, i} \in C_{j}$ and has label $\mathrm{r}$; this follows by the drawings of $G\left[F_{i}\right]$ and $G\left[F_{j}\right]$. Add $e_{i, j}$ and $e_{j, i}$ to $\mathcal{E}$.

For each $\overrightarrow{f_{i} f_{j}} \in A(\vec{T})$ with label r (Operation VII), choose $e_{i, j}$ in $E\left(C_{i}\right) \backslash \mathcal{E}$ with label r/sq and $e_{j, i}$ in $G\left[F_{j}\right]$ satisfying condition (ii) of Lemma 2.7. This is possible since $I^{\prime}$ has four edges with label r/sq. Note that $e_{j, i} \in C_{j}$ and has label r/sq; this follows by the drawings of $G\left[F_{i}\right]$ and $G\left[F_{j}\right]$. Add $e_{i, j}$ and $e_{j, i}$ to $\mathcal{E}$.

In the context of Operation IV, let $T^{\prime}$ be the subgraph of $T$ induced by edges with label $\mathrm{r}$. Each component of $T^{\prime}$ is a path since each $f_{i}$ is incident to at most two edges of $T$ with label r. Let $P$ be a component of $T^{\prime}$, and assume without loss of generality that $P=f_{0} f_{1} \cdots f_{m-1}$. For each $i, 0 \leq i \leq m-2$, starting at $i=0$, choose $e_{i, i+1}$ in $E\left(C_{i}\right) \backslash \mathcal{E}$ with label $\mathrm{r}$ and $e_{i+1, i}$ in $G\left[F_{i+1}\right]$ satisfying condition (ii) of Lemma 2.7. This is possible because the Hamilton cycle $\square$ has two edges labelled $\mathrm{r}$ in both rows two and four. Note that $e_{i+1, i} \in C_{i+1}$ and has label r; this follows by the drawings of $G\left[F_{i}\right]$ and $G\left[F_{i+1}\right]$. Add $e_{i, i+1}$ and $e_{i+1, i}$ to $\mathcal{E}$.

(6) For each $\overrightarrow{f_{i} f_{j}} \in A(\vec{T})$ with label c (Operations VI, VII and VIII), choose $e_{i, j}$ in $C_{i}$ with label c and $e_{j, i}$ in $G\left[F_{j}\right]$ that satisfies condition (ii) of Lemma 2.7. Note that $e_{j, i} \in C_{j}$ and has label c; this follows by the drawings of $G\left[F_{i}\right]$ and $G\left[F_{j}\right]$. Add $e_{i, j}$ and $e_{j, i}$ to $\mathcal{E}$.

For each $\overrightarrow{f_{i} f_{j}} \in A(\vec{T})$ with label c (Operation IX), choose $e_{i, j}$ in $E\left(C_{i}\right) \backslash \mathcal{E}$ with label c/e and $e_{j, i}$ in $G\left[F_{j}\right]$ satisfying condition (ii) of Lemma 2.7. This is possible because the Hamilton cycle $\sqsubset^{\prime}$ has two edges labelled c/e in columns two and four, and $f_{i}$ is incident to at most one edge in $T$ with label $\mathrm{c}$ and at most one edge in $T$ with label e. Note that $e_{j, i} \in C_{j}$ and has label c/e; this follows by the drawings of $G\left[F_{i}\right]$ and $G\left[F_{j}\right]$. Add $e_{i, j}$ and $e_{j, i}$ to $\mathcal{E}$.

In the context of Operations IV and V, let $T^{\prime}$ be the subgraph of $T$ induced by edges with label c. Each component of $T^{\prime}$ is a path since each $f_{i}$ is incident to at most two edges of $T$ with label c. Let $P$ be a component of $T^{\prime}$, and assume without loss of generality that $P=f_{0} f_{1} \cdots f_{m-1}$. For each $i, 0 \leq i \leq m-2$, starting at $i=0$, choose $e_{i, i+1}$ in $E\left(C_{i}\right) \backslash \mathcal{E}$ with label c and $e_{i+1, i}$ in $G\left[F_{i+1}\right]$ satisfying condition 
(ii) of Lemma 2.7. This is possible because the Hamilton cycles $\square$ and $\sqsubset$ have two edges labelled $\mathrm{c}$ in both columns two and four. Note that $e_{i+1, i} \in C_{i+1}$ and has label c; this follows by the drawings of $G\left[F_{i}\right]$ and $G\left[F_{i+1}\right]$. Add $e_{i, i+1}$ and $e_{i+1, i}$ to $\mathcal{E}$.

(7) For each $\overrightarrow{f_{i} f_{j}} \in A(\vec{T})$ with label pm, choose $e_{i, j}$ in $E\left(C_{i}\right) \backslash \mathcal{E}$ and $e_{j, i}$ in $E\left(G\left[F_{j}\right]\right) \backslash$ $\mathcal{E}$ satisfying condition (ii) of Lemma 2.7. Add $e_{i, j}$ and $e_{j, i}$ to $\mathcal{E}$.

Now $e_{i, j}$ and $e_{j, i}$ have been chosen for all $\overrightarrow{f_{i} f_{j}} \in A(\vec{T})$. By construction, (i) and (ii) of Lemma 2.7 are satisfied, and for each $G\left[F_{i}\right], 0 \leq i \leq N-1$, the Hamilton cycle $C_{i}$ satisfies condition (iii) of Lemma 2.7. Therefore, $G$ has a Hamilton cycle.

As a consequence of Lemmas 3.3, 3.5, 6.3 and 6.4, we now have our main result.

Theorem 1.1. If $H$ is a 2-tree then $k_{0}(H)=4$, unless $H \cong T \vee\{u\}$ for some tree $T$ and vertex $u$, where $T$ is a star on at least three vertices or the bipartition of $V(T)$ has two even parts; in these cases, $k_{0}(H)=5$.

As pointed out in Section 1, if $H$ is a $k$-tree then $k+2 \leq k_{0}(H) \leq k+3$. For both 1 trees (i.e., trees) and 2-trees, equality can occur in both the upper and lower bound. By [6, Corollary 5.6] and Theorem 1.1, if $H$ is a tree or 2-tree of diameter at least three, then the lower bound holds. We ask if this extends to $k$-trees, that is, if $H$ is a $k$-tree with diameter at least three, is it the case that $k_{0}(H)=k+2$ ? On a related note, $k$-trees are a subclass of chordal graphs. We ask if the techniques presented here can be extended to determine the Gray code numbers of other chordal graphs.

\section{References}

[1] J. A. Bondy and U. S. R. Murty, Graph Theory, volume 244 of Graduate Texts in Mathematics, Springer, New York, 2008, doi:10.1007/978-1-84628-970-5.

[2] P. Bonsma and L. Cereceda, Finding paths between graph colourings: PSPACE-completeness and superpolynomial distances, Theoret. Comput. Sci. 410 (2009), 5215-5226, doi:10.1016/j. tcs.2009.08.023.

[3] M. Celaya, K. Choo, G. MacGillivray and K. Seyffarth, Reconfiguring $k$-colourings of complete bipartite graphs, Kyungpook Math. J. 56 (2016), 647-655, doi:10.5666/kmj.2016.56.3. 647.

[4] L. Cereceda, J. van den Heuvel and M. Johnson, Connectedness of the graph of vertexcolourings, Discrete Math. 308 (2008), 913-919, doi:10.1016/j.disc.2007.07.028.

[5] C. C. Chen and N. F. Quimpo, On strongly hamiltonian abelian group graphs, in: K. L. McAvaney (ed.), Combinatorial Mathematics VIII, Springer, Berlin, volume 884 of Lecture Notes in Mathematics, pp. 23-34, 1981, doi:10.1007/bfb0091805, proceedings of the Eighth Australian Conference held at Deakin University, Geelong, August 25 - 29, 1980.

[6] K. Choo and G. MacGillivray, Gray code numbers for graphs, Ars Math. Contemp. 4 (2011), 125-139, doi:10.26493/1855-3974.196.0df.

[7] T. Dvořák, Hamiltonian cycles with prescribed edges in hypercubes, SIAM J. Discrete Math. 19 (2005), 135-144, doi:10.1137/s0895480103432805.

[8] M. Dyer, A. Flaxman, A. Frieze and E. Vigoda, Randomly coloring sparse random graphs with fewer colors than the maximum degree, Random Structures Algorithms 29 (2006), 450-465, doi:10.1002/rsa.20129. 
[9] S. Fallat and L. Hogben, Minimum rank, maximum nullity, and zero forcing number of graphs, in: L. Hogben (ed.), Handbook of Linear Algebra, Chapman \& Hall/CRC, New York, Second Edition, 2nd edition, 2013, doi:10.1201/b16113.

[10] S. Finbow and G. MacGillivray, Hamiltonicity of Bell and Stirling colour graphs, preprint.

[11] R. Haas, The canonical coloring graph of trees and cycles, Ars Math. Contemp. 5 (2012), 149157, doi:10.26493/1855-3974.168.464.

[12] R. Haas and K. Seyffarth, The $k$-dominating graph, Graphs Combin. 30 (2014), 609-617, doi: 10.1007/s00373-013-1302-3.

[13] R. Haas and K. Seyffarth, Reconfiguring dominating sets in some well-covered and other classes of graphs, Discrete Math. 340 (2017), 1802-1817, doi:10.1016/j.disc.2017.03.007.

[14] T. Ito, E. D. Demaine, N. J. A. Harvey, C. H. Papadimitriou, M. Sideri, R. Uehara and Y. Uno, On the complexity of reconfiguration problems, Theoret. Comput. Sci. 412 (2011), 1054-1065, doi:10.1016/j.tcs.2010.12.005.

[15] T. Ito, M. Kamiński and E. D. Demaine, Reconfiguration of list edge-colorings in a graph, Discrete Appl. Math. 160 (2012), 2199-2207, doi:10.1016/j.dam.2012.05.014.

[16] M. Jerrum, A very simple algorithm for estimating the number of $k$-colorings of a low-degree graph, Random Structures Algorithms 7 (1995), 157-165, doi:10.1002/rsa.3240070205.

[17] G. J. Simmons, Almost all $n$-dimensional rectangular lattices are Hamilton-laceable, in: F. Hoffman, D. McCarthy, R. C. Mullin and R. G. Stanton (eds.), Proceedings of the Ninth Southeastern Conference on Combinatorics, Graph Theory, and Computing, Utilitas Mathematica Publishing, Winnipeg, Manitoba, volume XXI of Congressus Numerantium, pp. 649661, 1978, held at Florida Atlantic University, Boca Raton, Florida, January 30 - February 2, 1978.

[18] A. Suzuki, A. E. Mouawad and N. Nishimura, Reconfigurations of dominating sets, in: Z. Cai, A. Zelikovsky and A. Bourgeois (eds.), Computing and Combinatorics, Springer, Cham, volume 8591 of Lecture notes in Computer Science, pp. 405-416, 2014, doi:10.1007/ 978-3-319-08783-2_35, proceedings of the 20th International Conference (COCOON 2014) held in Atlanta, GA, August 4 - 6, 2014. 


\section{Appendix A 3-colouring graphs of trees}

A 2-tree with a dominating vertex $u$ has the form $T \vee\{u\}$ for some tree $T$. Lemma 3.5 characterizes when $k_{0}(T \vee\{u\})=5$. Its proof requires a technical result (Lemma 3.6) that we prove in this appendix. To do so, we introduce additional terminology along with structural results on the 3 -colouring graphs of trees.

Define $L_{i}$ to be an assignment of lists to the vertices of $T \vee\{u\}$ with $L_{i}(u):=\{i\}$ and $L_{i}(w):=\{1,2,3,4\}$ for $w \in V(T)$. Then $G_{L_{i}}(T \vee\{u\}) \cong G_{3}(T)$ and thus, $G_{4}(T \vee\{u\})$ can be partitioned into four copies of $G_{3}(T)$ with edges between pairs of copies. We prove that when $T$ has even number of vertices then $G_{3}(T)$ is bipartite. First, we prove a more general result for $G_{L}(H)$ where $H$ is a connected graph.

Lemma A.1. Let $H$ be a connected graph on $n$ vertices and $L$ an assignment of lists to the vertices of $H$ such that $L(v) \subseteq\{1,2,3\}$ for each $v \in V(H)$. If $H$ is $L$-colourable and there is a vertex $w \in V(H)$ with $|L(w)|<3$, then $G_{L}(H) \subseteq Q_{n}$. In particular, $G_{L}(H)$ is a bipartite graph.

Proof. The proof is by induction on $n$. Observe that the result is true for $n=1$ and $n=2$.

First suppose that $|L(w)|=1$ and without loss of generality, $L(w)=\{1\}$. In any list colouring of $H$, each vertex $v \in N_{H}(w)$ cannot be coloured ' 1 '. Thus, let $\hat{L}$ be an assignment of lists of allowable colours defined as

$$
\hat{L}(v):= \begin{cases}L(v) \backslash\{1\}, & \text { if } v \in N_{H}(w), \\ L(v), & \text { otherwise. }\end{cases}
$$

Denote the components of $H-w$ by $H_{1}, H_{2}, \ldots, H_{N}$. Then by the inductive hypothesis, for each $i, 1 \leq i \leq N$,

$$
G_{\hat{L}}\left(H_{i}\right) \subseteq Q_{\left|V\left(H_{i}\right)\right|}
$$

Since $w$ must be coloured using the colour ' 1 ', we have

$$
\begin{array}{rlr}
G_{L}(H) & =G_{\hat{L}}(H-w) & \\
& =G_{\hat{L}}\left(\cup_{i=1}^{N} H_{i}\right) & \\
& =\square_{i=1}^{N} G_{\hat{L}}\left(H_{i}\right), & \\
& \subseteq \square_{i=1}^{N} Q_{\left|V\left(H_{i}\right)\right|} & \text { by Remark } 2.4 \\
& =Q_{n-1} . &
\end{array}
$$

Next suppose that $|L(w)|=2$ and without loss of generality, $L(w):=\{1,2\}$. For $i=1,2$, define $L_{i}$ as $L_{i}(w):=\{i\}$ and $L_{i}(v):=L(v)$ for $v \neq w$ with $v \in V(H)$. Also define $\hat{L}_{i}$ as $\hat{L}_{i}(w):=\{i\}$ and $\hat{L}_{i}(v):=\{1,2,3\}$ for $v \neq w$ with $v \in V(H)$. Observe that $G_{L_{1}}(H) \subseteq G_{\hat{L}_{1}}(H)$ and $G_{L_{2}}(H) \subseteq G_{\hat{L}_{2}}(H)$. Furthermore, $G_{\hat{L}_{1}}(H) \cong G_{\hat{L}_{2}}(H)$, and hence, $G_{L}(H) \subseteq G_{\hat{L}_{1}}(H) \square K_{2}$. By the preceding argument, $G_{\hat{L}_{1}}(H) \subseteq Q_{n-1}$, and therefore

$$
G_{L}(H) \subseteq Q_{n-1} \square K_{2}=Q_{n}
$$

Lemma A.2. If $T$ is a tree with an even number of vertices then $G_{3}(T)$ is bipartite. 
Proof. If $T \cong K_{2}$, then $G_{3}(T) \cong C_{6}$. Let $T$ be a tree on an even number of vertices with $|V(T)| \geq 4$ having bipartition $(A, B)$. Fix $u \in A, v \in B$ with $u$ adjacent to $v$ in $T$. Define $\mathcal{H}:=G_{3}(T)$. For each $1 \leq i \neq j \leq 3$ let

$$
V_{i j}:=\{c \in V(\mathcal{H}) \mid c(u)=i \text { and } c(v)=j\} .
$$

Then $\left\{V_{12}, V_{13}, V_{23}, V_{21}, V_{31}, V_{32}\right\}$ is a partition of $V(\mathcal{H})$. Observe each $\mathcal{H}\left[V_{i j}\right]$ is connected (this can be shown by induction on $|V(T)|$ ), and that $\left[V_{\alpha \beta}, V_{\gamma \delta}\right] \neq \emptyset$ if and only if $\alpha=\gamma$ or $\beta=\delta$. It follows that $\mathcal{H}_{1}=\mathcal{H}\left[V_{12} \cup V_{13} \cup V_{23} \cup V_{21}\right]$ is connected, and by Lemma A.1 is bipartite. Similarly, $\mathcal{H}_{2}=\mathcal{H}\left[V_{23} \cup V_{21} \cup V_{31} \cup V_{32}\right]$ and $\mathcal{H}_{3}=$ $\mathcal{H}\left[V_{31} \cup V_{32} \cup V_{12} \cup V_{13}\right]$ are connected and bipartite. Denote the two-coloured vertices of $\mathcal{H}$ (that is, the colourings of $T$ with two colours) by $c_{i j} \in V_{i j}$ such that

$$
c_{i j}(x):= \begin{cases}i, & \text { if } x \in A, \\ j, & \text { if } x \in B .\end{cases}
$$

Suppose the bipartition of each $\mathcal{H}\left[V_{i j}\right]$ is $\left(A_{i j}, B_{i j}\right)$ where $c_{i j} \in B_{i j}$.

If $|A|$ and $|B|$ are even, then $d_{\mathcal{H}}\left(c_{i j}, c_{i^{\prime} j^{\prime}}\right)$ is even. It follows that

$$
\left(A_{12} \cup A_{13} \cup A_{23} \cup A_{21}, B_{12} \cup B_{13} \cup B_{23} \cup B_{21}\right)
$$

is a bipartition of $\mathcal{H}_{1}$,

$$
\left(A_{23} \cup A_{21} \cup A_{31} \cup A_{32}, B_{23} \cup B_{21} \cup B_{31} \cup B_{32}\right)
$$

is a bipartition of $\mathcal{H}_{2}$, and

$$
\left(A_{31} \cup A_{32} \cup A_{12} \cup A_{13}, B_{31} \cup B_{32} \cup B_{12} \cup B_{13}\right)
$$

is a bipartition of $\mathcal{H}_{3}$. Hence,

$$
\mathcal{A}:=\bigcup_{1 \leq i \neq j \leq 3} A_{i j} \quad \text { and } \mathcal{B}:=\bigcup_{1 \leq i \neq j \leq 3} B_{i j}
$$

are independent, and thus form a bipartition of $\mathcal{H}$.

If $|A|$ and $|B|$ are both odd, $d_{\mathcal{H}}\left(c_{i j}, c_{i^{\prime} j^{\prime}}\right)$ is even if and only if $i \neq i^{\prime}$ and $j \neq j^{\prime}$. It follows that

$$
\left(A_{12} \cup B_{13} \cup A_{23} \cup B_{21}, B_{12} \cup A_{13} \cup B_{23} \cup A_{21}\right)
$$

is a bipartition of $\mathcal{H}_{1}$,

$$
\left(A_{23} \cup B_{21} \cup A_{31} \cup B_{32}, B_{23} \cup A_{21} \cup B_{31} \cup A_{32}\right)
$$

is a bipartition of $\mathcal{H}_{2}$, and

$$
\left(A_{31} \cup B_{32} \cup A_{12} \cup B_{13}, B_{31} \cup A_{32} \cup B_{12} \cup A_{13}\right)
$$

is a bipartition of $\mathcal{H}_{3}$. Hence,

$$
\mathcal{A}=\left\{A_{12} \cup B_{13} \cup A_{23} \cup B_{21} \cup A_{31} \cup B_{32}\right\}
$$

and

$$
\mathcal{B}=\left\{B_{12} \cup A_{13} \cup B_{23} \cup A_{21} \cup B_{31} \cup A_{32}\right\}
$$

are independent, and thus form a bipartition of $\mathcal{H}$. 
Definition A.3. A connected bipartite graph with bipartition $(A, B)$ is Hamilton laceable if there is a Hamilton path between any $u \in A$ and $v \in B$.

Remark A.4. The following are Hamilton laceable.

1. $P_{2 k_{1}} \square P_{k_{2}} \square P_{k_{3}} \square \cdots \square P_{k_{n}}$, for $n \geq 2$ and $k_{i} \geq 1,1 \leq i \leq n$ [17].

2. $Q_{n}$, for $n \geq 1[5]$.

Definition A.5. A $B$-graph with vertex partition $\left\{F_{0}, F_{1}, \ldots, F_{N-1}\right\}$ is a bipartite graph $G$ with bipartition $(\mathcal{A}, \mathcal{B})$ together with a partition $\left\{F_{0}, F_{1}, \ldots, F_{N-1}\right\}$ of $V(G)$ so that, for $i=0,1, \ldots, N-1, G\left[F_{i}\right]$ is Hamilton laceable.

Lemma A.6. Let $G$ be a $B$-graph with vertex partition $\left\{F_{0}, F_{1}, \ldots, F_{N-1}\right\}$ and bipartition $(\mathcal{A}, \mathcal{B})$. Suppose for each $i=1,2, \ldots, N-1$, there is an edge $b_{i-1} a_{i}$ with $b_{i-1} \in$ $\mathcal{B} \cap F_{i-1}$ and $a_{i} \in \mathcal{A} \cap F_{i}$. Then $G$ has a Hamilton path between any vertex in $\mathcal{A} \cap F_{0}$ and any vertex in $\mathcal{B} \cap F_{N-1}$.

Proof. Let $a_{0} \in \mathcal{A} \cap F_{0}$ and $b_{N-1} \in \mathcal{B} \cap F_{N-1}$. For each $F_{i}, i=0,1, \ldots, N-1$, choose a Hamilton path $P_{i}$ in $G\left[F_{i}\right]$ between $b_{i}$ and $a_{i}$. Then

$$
\left(\bigcup_{i=0}^{N-2}\left\{b_{i} a_{i+1}\right\}\right) \cup\left(\bigcup_{i=0}^{N-1} E\left(P_{i}\right)\right)
$$

are the edges of a Hamilton path in $G$ between $a_{0}$ and $b_{N-1}$.

Corollary A.7. Let $G$ be a $B$-graph with vertex partition $\left\{F_{0}, F_{1}, \ldots, F_{N-1}\right\}$ and bipartition $(\mathcal{A}, \mathcal{B})$ such that $\left[F_{i-1}, F_{i}\right]$ is a set of independent edges and $\left|\left[F_{i-1}, F_{i}\right]\right| \geq 2$, $i=1,2, \ldots, N-1$. If for each $i=1,2, \ldots, N-1$, the endpoints of any pair of edges in $\left[F_{i-1}, F_{i}\right]$ induces a 4 -cycle in $G$, then $G$ has a Hamilton path between any vertex in $\mathcal{A} \cap F_{0}$ and any vertex in $\mathcal{B} \cap F_{N-1}$.

Proof. Let $a_{0} \in \mathcal{A} \cap F_{0}$ and $b_{N-1} \in \mathcal{B} \cap F_{N-1}$. For each $\left[F_{i-1}, F_{i}\right], i=1,2, \ldots$, $N-1$, choose two edges $b_{i-1} a_{i}$ and $b_{i-1}^{\prime} a_{i}^{\prime}$. Then $G\left[\left\{a_{i}, a_{i}^{\prime}, b_{i-1}, b_{i-1}^{\prime}\right\}\right]$ induces a 4cycle $a_{i} a_{i}^{\prime} b_{i-1}^{\prime} b_{i-1} a_{i}$. Note that either $b_{i-1} \in \mathcal{B}$ or $b_{i-1}^{\prime} \in \mathcal{B}$. Without loss of generality, suppose $b_{i-1} \in \mathcal{B}$. Then $a_{i} \in \mathcal{A}$. The result follows by Lemma A.6.

Definition A.8. An odd flare is a tree obtained from $K_{1, t}, t \geq 3$ and odd, by a single subdivision of one edge.

Lemma 3.6. Let $T$ be a tree with bipartition $(A, B)$, where $|A|:=\ell$ and $|B|:=r$, and let $G_{3}(T)$ be the 3-colouring graph of $T$ with colours $C=\{1,2,3\}$. Define $c_{i j}$ to be the vertex of $G_{3}(T)$ with $c_{i j}(a)=i$ for all $a \in A$ and $c_{i j}(b)=j$ for all $b \in B$.

(1) If $\ell, r>0$ are both even, then $G_{3}(T)$ has no spanning subgraph consisting only of paths whose ends are in $\left\{c_{12}, c_{13}, c_{21}, c_{23}, c_{31}, c_{32}\right\}$.

(2) If $\ell>1$ is odd and $r>0$ is even, then $G_{3}(T)$ has a Hamilton path from $c_{12}$ to $c_{23}$.

(3) If $\ell>1$ and $r>1$ are both odd, then $G_{3}(T)$ has a Hamilton path from $c_{12}$ to $c_{13}$. 
Proof. (1): Suppose $\ell, r>0$ are both even. By Lemma A.2, $\mathcal{H}:=G_{3}(T)$ is bipartite. Suppose $\mathcal{H}$ has bipartition $(\mathcal{A}, \mathcal{B})$. Without loss of generality, assume $c_{12} \in \mathcal{A}$. Since $d_{\mathcal{H}}\left(c_{12}, c_{i j}\right)$ is even for each $c_{i j} \in\left\{c_{12}, c_{13}, c_{21}, c_{23}, c_{31}, c_{32}\right\}$ and $\mathcal{H}$ is bipartite, we have $\left\{c_{12}, c_{13}, c_{21}, c_{23}, c_{31}, c_{32}\right\} \subseteq \mathcal{A}$. By [6, Theorem 5.5] there is a Hamilton cycle in $\mathcal{H}$, and thus $|\mathcal{A}|=|\mathcal{B}|$. It follows that there is no spanning subgraph of $\mathcal{H}$ consisting only of paths whose ends are in $\left\{c_{12}, c_{13}, c_{21}, c_{23}, c_{31}, c_{32}\right\}$ (otherwise $|\mathcal{A}|>|\mathcal{B}|$ ).

(2): Suppose $\ell>1$ is odd and $r>0$ is even. Then $\ell+r \geq 5$. We first prove that $G_{3}(T)$ has a Hamilton path between $c_{12}$ and $c_{23}$ whenever $T$ is $P_{5}$ or any odd flare. If $T \cong P_{5}$ (with $|A|=3,|B|=2$ ), then there is a Hamilton path between $c_{12}$ to $c_{23}$ in $G_{3}\left(P_{5}\right)$, as described in Figure 15. A 3-colouring $f$ of $P_{5}=x_{1} x_{2} x_{3} x_{4} x_{5}$ is represented by the string $f\left(x_{1}\right) f\left(x_{2}\right) f\left(x_{3}\right) f\left(x_{4}\right) f\left(x_{5}\right)$; for example, $c_{12}=12121$ and $c_{23}=23232$.

$\begin{array}{llllll}12121 & 21313 & 32321 & 13131 & 23231 & 31212 \\ 12321 & 21323 & 32121 & 12131 & 13231 & 31232 \\ 12323 & 21321 & 32123 & 32131 & 13232 & 31231 \\ 12313 & 31321 & 12123 & 32132 & 13212 & 21231 \\ 12312 & 31323 & 13123 & 12132 & 13213 & 21232 \\ 32312 & 31313 & 23123 & 13132 & 23213 & 21212 \\ 31312 & 32313 & 23121 & 23132 & 21213 & 23212 \\ 21312 & 32323 & 13121 & 23131 & 31213 & 23232\end{array}$

Figure 15: A Hamilton path in $G_{3}\left(P_{5}\right)$ from $c_{12}$ to $c_{23}$.

Let $T$ be an odd flare on $n$ vertices with $u$ denoting the unique vertex of degree two and let $N_{T}(u)=\left\{v, v^{\prime}\right\}$ where $v$ is the unique vertex of degree $n-2$ (Figure 16).

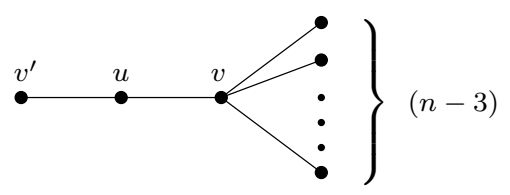

Figure 16: An odd flare $T$.

Here $|A|=n-2$ and $|B|=2$. We partition $\mathcal{H}:=G_{3}(T)$ according to the colours of $u$ and $v$. For each $1 \leq i \neq j \leq 3$, let $V_{i j}:=\{c \in V(\mathcal{H}) \mid c(u)=i$ and $c(v)=j\}$ and let $L_{i j}$ be an assignment of lists to the vertices of $H$ such that $L_{i j}(u):=\{i\}, L_{i j}(v):=\{j\}$ and $L_{i j}(w):=\{1,2,3\}$ for $w \in V(H-\{u, v\})$. Note that $G_{L_{i j}}(H) \cong \mathcal{H}\left[V_{i j}\right] \cong Q_{n-2}$, for each $1 \leq i \neq j \leq 3$. Let $\mathcal{H}_{1}:=\mathcal{H}\left[V_{32} \cup V_{12}\right] \cong Q_{n-3} \square P_{4}, \mathcal{H}_{4}:=\mathcal{H}\left[V_{21} \cup V_{31}\right] \cong$ $Q_{n-3} \square P_{4}, \mathcal{H}_{2}:=\mathcal{H}\left[V_{13}\right]$, and $\mathcal{H}_{3}:=\mathcal{H}\left[V_{23}\right]$.

For $\{i, j, k\}=\{1,2,3\}$, let $d_{i j k} \in V(\mathcal{H})$ denote the vertex with $d_{i j k}(v)=j$, $d_{i j k}\left(v^{\prime}\right)=k$, and $d_{i j k}(w)=i$ for all $w \in N_{T}(v)$. Then $\left[V_{12}, V_{13}\right]=\left\{c_{12} d_{132}, d_{123} c_{13}\right\}$ and $\left[V_{23}, V_{21}\right]=\left\{c_{21} d_{231}, d_{213} c_{23}\right\}$.

Claim. For $m \geq 2$, every edge of $Q_{m} \square P_{4}$ is in a Hamilton cycle of $Q_{m} \square P_{4}$.

The claim follows by induction and the fact that any pair of distinct edges of $Q_{m-1}$ ( $m \geq 3$ ) belongs to a Hamilton cycle of $Q_{m-1}$ (for example, see [7, Theorem 4.1]).

In what follows, we define cycles and paths to construct a Hamilton path from $c_{12}$ to $c_{23}$ in $G_{3}(T)$ when $T$ is an odd flare on $n$ vertices. See Figure 17 for the case $n=5$; here, 
the labels of the two columns of $V_{i j}$ represent the colour choices for $v^{\prime}$ and the labels of the rows of $V_{i j}$ represent the colour choices for the two vertices in $N_{T}(v) \backslash\{u\}$. Let $C_{1}$ be a Hamilton cycle of $\mathcal{H}_{1}$ containing $c_{12} d_{123}$ and $C_{4}$ be a Hamilton cycle of $\mathcal{H}_{4}$ containing $d_{213} c_{21}$; these exist by the previous claim. Let $c \in N_{\mathcal{H}_{3}}\left(c_{23}\right)$ have $c\left(v^{\prime}\right)=3$ and $d \in V_{13}$ be the unique vertex of $\mathcal{H}_{2}$ adjacent to $c$. By [7, Theorem 4.1], there is a Hamilton cycle $C_{3}$ in $\mathcal{H}_{3}$ containing the edges $c c_{23}$ and $c_{23} d_{231}$. Observe $d_{\mathcal{H}_{2}}\left(d, c_{13}\right)$ is odd. Since $\mathcal{H}_{2}$ is Hamilton laceable, there is a Hamilton path $P$ between $d$ and $c_{13}$ (see Figure 17).

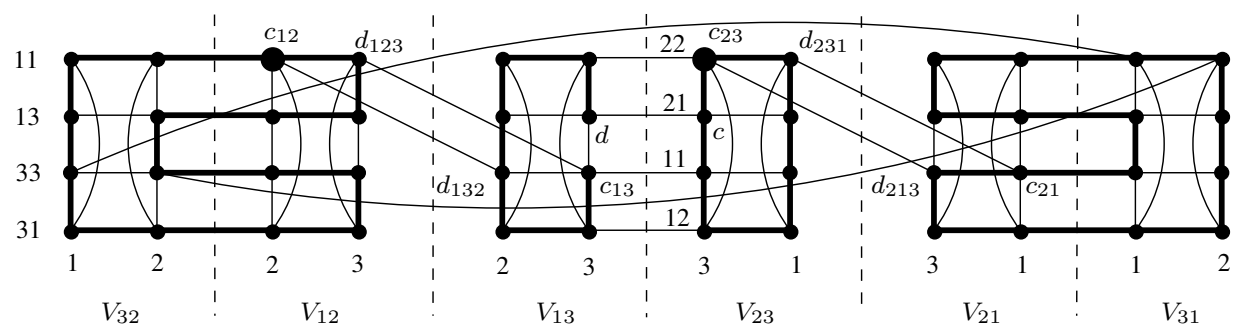

Figure 17: $\mathcal{H}=G_{3}(T), T$ an odd flare on five vertices, along with $C_{1}, P, C_{3}$, and $C_{4}$.

Now,

$$
\begin{aligned}
\left(C_{1}-\left\{c_{12} d_{123}\right\}\right) \cup\left\{d_{123} c_{13}\right\} \cup P \cup & \{d c\} \cup\left(C_{3}-\left\{c_{23} c, c_{23} d_{231}\right\}\right) \\
& \cup\left\{d_{231} c_{21}\right\} \cup\left(C_{4}-\left\{c_{21} d_{213}\right\}\right) \cup\left\{d_{213} c_{23}\right\}
\end{aligned}
$$

is a Hamilton path in $\mathcal{H}$ between $c_{12}$ and $c_{23}$ (see Figure 18 for $n=5$ ).

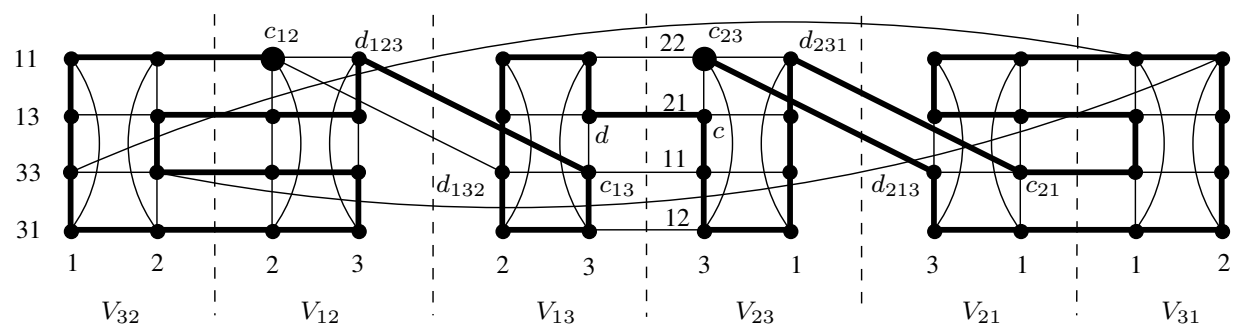

Figure 18: A Hamilton path between $c_{12}$ and $c_{23}$ in $\mathcal{H}=G_{3}(T), T$ an odd flare on five vertices.

Now suppose $T$ is a tree on $n>5$ vertices with $n$ odd that is not isomorphic to a star or an odd flare. Then there are leaves $x, y \in V(T)$ with $d_{T}(x, y) \geq 3$. By choosing leaves $x$ and $y$ so that $d_{T}(x, y) \geq 3$ is minimum, $T^{\prime}:=T-\{x, y\}$ is not a star. Let $N_{T}(x):=\left\{x^{\prime}\right\}$ and $N_{T}(y):=\left\{y^{\prime}\right\}$; since $d_{T}(x, y) \geq 3, x^{\prime} \neq y^{\prime}$.

Let $\left(A^{\prime}, B^{\prime}\right)$ denote the bipartition of $T^{\prime}$ with $A^{\prime} \subseteq A, B^{\prime} \subseteq B$, and define $c_{i j}^{\prime}$ to be the vertex of $\mathcal{H}^{\prime}:=G_{3}\left(T^{\prime}\right)$ with $c_{i j}^{\prime}(a)=i$ for all $a \in A^{\prime}$ and $c_{i j}^{\prime}(b)=j$ for all $b \in B^{\prime}$. By the inductive hypothesis, $\mathcal{H}^{\prime}$ has a Hamilton path between $c_{12}^{\prime}$ and $c_{23}^{\prime}$. Let $V\left(\mathcal{H}^{\prime}\right):=$ $\left\{f_{0}, f_{1}, \ldots, f_{N-1}\right\}$. Since $\mathcal{H}^{\prime}$ has a Hamilton path, we may assume that $f_{0} f_{1} \cdots f_{N-1}$ is a Hamilton path in $\mathcal{H}^{\prime}$ between $f_{0}=c_{12}^{\prime}$ and $f_{N-1}=c_{23}^{\prime}$. Since $c_{12}^{\prime}$ and $c_{23}^{\prime}$ differ in colour on at least two vertices, $f_{0}$ is not adjacent to $f_{N-1}$ in $\mathcal{H}^{\prime}$. 
For $0 \leq i \leq N-1$, let $F_{i}$ be the set of 3-colourings of $\mathcal{H}$ that agree with $f_{i}$ on $V\left(T^{\prime}\right)$. Then $\left\{F_{0}, F_{1}, \ldots, F_{N-1}\right\}$ is a partition of the vertices of $\mathcal{H}$, and $\mathcal{H}\left[F_{i}\right] \cong C_{4}$, $0 \leq i \leq N-1$. If $f_{i-1}$ and $f_{i}$ differ on the colour of a vertex of $V\left(T^{\prime}\right) \backslash\left\{x^{\prime}, y^{\prime}\right\}$, then $\left|\left[F_{i-1}, F_{i}\right]\right|=4$ and $\mathcal{H}\left[F_{i-1} \cup F_{i}\right] \cong Q_{3}$. Otherwise, $f_{i-1}$ and $f_{i}$ differ on the colour of $x^{\prime}$ or $y^{\prime}$, implying that $\left|\left[F_{i-1}, F_{i}\right]\right|=2$, the subgraph of $\mathcal{H}$ induced by the endpoints of the edges of $\left[F_{i-1}, F_{i}\right]$ is a 4 -cycle, and $\mathcal{H}\left[F_{i-1} \cup F_{i}\right] \cong P_{4} \square K_{2}$.

Consider the spanning subgraph $G$ of $\mathcal{H}$ with edge set

$$
\left(\bigcup_{i=0}^{N-1} E\left(\mathcal{H}\left[F_{i}\right]\right)\right) \cup\left(\bigcup_{i=1}^{N-1}\left[F_{i-1}, F_{i}\right]\right) .
$$

Note that $G$ is a connected $B$-graph. Let $(\mathcal{A}, \mathcal{B})$ be the bipartition of $G$ and assume $c_{12} \in \mathcal{A}$.

First suppose $c_{23} \in \mathcal{B}$. As $c_{12} \in F_{0}$ and $c_{23} \in F_{N-1}$, it follows from Corollary A.7 that there is a Hamilton path in $G$ between $c_{12}$ and $c_{23}$. Now suppose $c_{23} \in \mathcal{A}$. Since $d_{G}\left(c_{12}, c_{23}\right)$ is odd, $\mathcal{H}$ must have an edge $e$ with both endpoints in $\mathcal{A}$ or both endpoints in $\mathcal{B}$. Suppose $e \in\left[F_{p}, F_{q}\right]$, where $0 \leq p<q \leq N-1$. Since $f_{0}$ is not adjacent to $f_{N-1}$ in $\mathcal{H}^{\prime}$, either $p \neq 0$ or $q \neq N-1$. Without loss of generality we assume $p \neq 0$. Then $f_{p} f_{q} \in E\left(\mathcal{H}^{\prime}\right)$, and either

(i) $\left|\left[F_{p}, F_{q}\right]\right|=4$ and $\mathcal{H}\left[F_{p} \cup F_{q}\right] \cong Q_{3}$, or

(ii) $\left|\left[F_{p}, F_{q}\right]\right|=2$, the subgraph of $\mathcal{H}$ induced by the endpoints of the edges of $\left[F_{p}, F_{q}\right]$ is a 4-cycle, and $\mathcal{H}\left[F_{p} \cup F_{q}\right] \cong P_{4} \square K_{2}$.

In either case, there exists another edge $e^{\prime} \in\left[F_{p}, F_{q}\right]$ such that $e:=u v, e^{\prime}:=u^{\prime} v^{\prime}$, $u, u^{\prime} \in F_{p}$, and $u v v^{\prime} u^{\prime} u$ is a 4-cycle. The choice of $e=u v$ ensures that $u, v \in \mathcal{A}$ or $u, v \in \mathcal{B}$.

Consider the spanning tree $\widehat{T}$ of $\mathcal{H}^{\prime}$ with edge set

$$
E(\widehat{T}):=\left\{f_{i-1} f_{i} \mid 1 \leq i \leq q-1\right\} \cup\left\{f_{i-1} f_{i} \mid q+1 \leq i \leq N-1\right\} \cup\left\{f_{p} f_{q}\right\} .
$$

We define $J$ to be the spanning subgraph of $\mathcal{H}$ with edge set

$$
\left(\bigcup_{i=0}^{N-1} E\left(\mathcal{H}\left[F_{i}\right]\right)\right) \cup\left(\bigcup_{f_{i} f_{j} \in E(\widehat{T})}\left[F_{i}, F_{j}\right]\right)
$$

Then $J$ is a connected $B$-graph. Let $(\mathcal{K}, \mathcal{L})$ be the bipartition of $J$; we may assume that $c_{12} \in \mathcal{K}$. Since $f_{p} f_{q} \in E(\widehat{T}),\left[F_{p}, F_{q}\right] \subseteq E(J)$; in particular, $e=u v$ and $e^{\prime}=u^{\prime} v^{\prime}$ are edges of $J$, so $u$ and $v$ are in different parts of the partition $(\mathcal{K}, \mathcal{L})$, and thus $c_{23} \in \mathcal{L}$.

Case 1. If $\left|\left[F_{p}, F_{p+1}\right]\right|=\left|\left[F_{p}, F_{q}\right]\right|=2$, then $f_{p} f_{q}, f_{p} f_{p+1} \in E\left(\mathcal{H}^{\prime}\right)$ arise from colour changes on $x^{\prime}$ and $y^{\prime}$. Since there are only three possible vertex colours, there is only one possible colour that $x^{\prime}$ could change to, and only one possible colour that $y^{\prime}$ could change to; i.e., one of $f_{p} f_{q}, f_{p} f_{p+1}$ arises from a colour change on $x^{\prime}$ and the other from a colour change on $y^{\prime}$. Assuming that $\mathcal{H}\left[F_{p}\right]$ is the 4-cycle $u u^{\prime} w w^{\prime} u$, it follows that $u, u^{\prime}$ are incident to the edges of $\left[F_{p}, F_{q}\right]$ and that without loss of generality $u^{\prime}, w$ are incident to the edges of $\left[F_{p}, F_{p+1}\right]$. 
Let $\left[F_{p}, F_{q}\right]=\left\{u v, u^{\prime} v^{\prime}\right\}$ and $\left[F_{p}, F_{p+1}\right]=\left\{w z, u^{\prime} z^{\prime}\right\}$. Since $u u^{\prime} \in E(J)$, exactly one of $u, u^{\prime}$ is in $\mathcal{L}$.

Case 1(a). First suppose that $u \in \mathcal{L}$. Let $J_{1}$ denote the subgraph of $J$ induced by $\cup_{i=0}^{p} F_{i}$. Then $J_{1}$ is a $B$-graph with vertex partition $\left\{F_{0}, F_{1}, \ldots, F_{p}\right\}$ and bipartition $(\mathcal{K}, \mathcal{L})$ satisfying the conditions of Corollary A.7, with $c_{12} \in \mathcal{K} \cap F_{0}$ and $u \in \mathcal{L} \cap F_{p}$. Thus $J_{1}$ has a Hamilton path $R_{1}$ between $c_{12}$ and $u$. Note that the proof of Corollary A.7 implies that $R_{1}$ can be constructed so as to contain the edge $u^{\prime} w$.

Let $J_{2}$ be the subgraph of $J$ induced by $\cup_{i=q}^{N-1} F_{i}$. Then $J_{2}$ is a $B$-graph with vertex partition $\left\{F_{q}, F_{q+1}, \ldots, F_{N-1}\right\}$ and bipartition $(\mathcal{K}, \mathcal{L})$ satisfying the conditions of Corollary A.7, with $v \in \mathcal{K} \cap F_{q}$ and $c_{23} \in \mathcal{L} \cap F_{N-1}$. Thus $J_{2}$ has a Hamilton path $R_{2}$ between $v$ and $c_{23}$.

Finally, let $J_{3}$ be the subgraph of $J$ induced by $\cup_{i=p}^{q-1} F_{i}$. Then by Lemma $2.7, J_{3}$ has a Hamilton cycle $C$ containing the edges $u u^{\prime}, w w^{\prime}$ and $u w^{\prime}$. Let $R_{3}$ be the path between $u^{\prime}$ and $w$ obtained by deleting $u$ and $w^{\prime}$ from $C$.

Now concatenate paths $R_{1}, R_{2}, R_{3}$ and delete edge $u^{\prime} w \in R_{1}$ to form a Hamilton path between $c_{12}$ and $c_{23}$.

Case 1(b). Now suppose that $u \in \mathcal{K}$. Then $u^{\prime} \in \mathcal{L}$. Since $p \geq 1$ and $\left|\left[F_{p}, F_{p+1}\right]\right|=$ $\left|\left[F_{p}, F_{q}\right]\right|=2$, we have $\left|\left[F_{p-1}, F_{p}\right]\right|=4$. Let $t \in F_{p-1}$ be such that $t u \in\left[F_{p-1}, F_{p}\right]$. Then $t \in \mathcal{L}$.

Let $J_{1}$ denote the subgraph of $J$ induced by $\cup_{i=0}^{p-1} F_{i}$. Then $J_{1}$ is a $B$-graph with vertex partition $\left\{F_{0}, F_{1}, \ldots, F_{p-1}\right\}$ and bipartition $(\mathcal{K}, \mathcal{L})$ satisfying the conditions of Corollary A.7, with $c_{12} \in \mathcal{K} \cap F_{0}$ and $t \in \mathcal{L} \cap F_{p-1}$. Thus $J_{1}$ has a Hamilton path $R_{0}$ between $c_{12}$ and $t$. Let $R_{1}$ be the concatenation of paths $R_{0}$ and $t u w^{\prime} w u^{\prime}$.

We define $J_{2}$ and $R_{3}$ as in Case 1(a). The same argument with $v^{\prime}$ in place of $v$ gives a Hamilton path $R_{2}$ between $v^{\prime}$ and $c_{23}$. Now concatenate paths $R_{1}, R_{2}, R_{3}$ and delete edge $u^{\prime} w \in R_{1}$ to form a Hamilton path between $c_{12}$ and $c_{23}$.

Case 2. Suppose $\left|\left[F_{p}, F_{q}\right]\right|=4$ and label the 4-cycle of $\mathcal{H}\left[F_{p}\right]$ as $u u^{\prime} w w^{\prime} u$. Let $J_{3}$ be the subgraph of $J$ induced by $\cup_{i=p}^{q-1} F_{i}$. Then by Lemma 2.7, $J_{3}$ has a Hamilton cycle $C$. Without loss of generality, suppose $C$ contains the edges $u u^{\prime}, w w^{\prime}$ and $u w^{\prime}$. Let $R_{3}$ be the path between $u^{\prime}$ and $w$ obtained by deleting $u$ and $w^{\prime}$ from $C$.

Let $J_{1}$ denote the subgraph of $J$ induced by $\cup_{i=0}^{p} F_{i}$. Then $J_{1}$ is a $B$-graph with vertex partition $\left\{F_{0}, F_{1}, \ldots, F_{p}\right\}$ and bipartition $(\mathcal{K}, \mathcal{L})$ satisfying the conditions of Corollary A.7, with $c_{12} \in \mathcal{K} \cap F_{0}$. Thus $J_{1}$ has Hamilton paths $R_{1}^{\prime}$ and $R_{1}^{\prime \prime}$ between $c_{12}$ and the two vertices in $\mathcal{L} \cap F_{p}$. Let $R_{1}$ be one of $R_{1}^{\prime}$ and $R_{1}^{\prime \prime}$ such that $R_{1}$ contains edge $u^{\prime} w$. Suppose $R_{1}$ is between $c_{12}$ and $t$. Then $t \in \mathcal{L}$. Let $t^{\prime} \in F_{q}$ be such that $t t^{\prime} \in\left[F_{p}, F_{q}\right]$. Then $t^{\prime} \in \mathcal{K}$.

We define $J_{2}$ as in Case 1(a). The same argument with $t^{\prime}$ in place of $v$ gives a Hamilton path $R_{2}$ between $t^{\prime}$ and $c_{23}$. Now concatenate paths $R_{1}, R_{2}, R_{3}$ and delete edge $u^{\prime} w \in R_{1}$ to form a Hamilton path between $c_{12}$ and $c_{23}$.

Case 3. Suppose $\left|\left[F_{p}, F_{p+1}\right]\right|=4$. Let $J_{1}$ denote the subgraph of $J$ induced by $\left(\cup_{i=0}^{p} F_{i}\right) \cup$ $\left(\cup_{i=q}^{N-1} F_{i}\right)$. Then $J_{1}$ is a $B$-graph with vertex partition

$$
\left\{F_{0}, F_{1}, \ldots, F_{p}, F_{q}, F_{q+1}, \ldots, F_{N-1}\right\}
$$

and bipartition $(\mathcal{K}, \mathcal{L})$ satisfying the conditions of Corollary A.7, with $c_{12} \in \mathcal{K} \cap F_{0}$ and $c_{23} \in \mathcal{L} \cap F_{N-1}$. Thus $J_{1}$ has a Hamilton path $R$ between $c_{12}$ and $c_{23}$. Note that the proof 
of Corollary A.7 implies that $R$ can be constructed so as to contain three edges of $\mathcal{H}\left[F_{p}\right]$.

Let $J_{3}$ be the subgraph of $J$ induced by $\cup_{i=p+1}^{q-1} F_{i}$. Then by Lemma $2.7, J_{3}$ has a Hamilton cycle $C$. Note that $C$ contains three edges of $\mathcal{H}\left[F_{p+1}\right]$.

By the Pigeonhole Principle there are $s, t \in F_{p}$ and $s^{\prime}, t^{\prime} \in F_{p+1}$ with $s t \in R, s^{\prime} t^{\prime} \in C$ such that $s s^{\prime} t t^{\prime} s$ is a 4-cycle in $\mathcal{H}\left[F_{p} \cup F_{p+1}\right]$. Now $(R \cup C)-\left\{s t, s^{\prime} t^{\prime}\right\}$ is a Hamilton path in $J$ between $c_{12}$ and $c_{23}$.

(3): Finally suppose, $\ell>1$ and $r>1$ are both odd. We define $\mathcal{E}_{s, t}^{k}$ to be the tree obtained from $P_{k}$ with ends $u$ and $v$ by appending $s$ leaves to $u$ and $t$ leaves to $v$. The proof is by induction and has the following base cases.

Base Case 1. We first prove that $\mathcal{H}:=G_{3}(T)$ has a Hamilton path between $c_{12}$ and $c_{13}$ when $T \cong \mathcal{E}_{0,0}^{4 k+2}$ for $k \geq 0$, that is, when $T \cong P_{4 k+2}$. If $T \cong P_{2}$ then $c_{12} c_{32} c_{31} c_{21} c_{23} c_{13}$ is such a Hamilton path. Suppose $k>0$ and $T \cong P_{4 k+2}$. Let $u$ and $v$ be the leaves of $T, N_{T}(u):=\left\{u^{\prime}\right\}, N_{T}(v):=\left\{v^{\prime}\right\}, N_{T}\left(u^{\prime}\right):=\left\{u, u^{\prime \prime}\right\}$ and $N_{T}\left(v^{\prime}\right):=\left\{v, v^{\prime \prime}\right\}$. Then $T^{\prime}:=T-\left\{u, u^{\prime}, v, v^{\prime}\right\}$ is isomorphic to $P_{4(k-1)+2}$. Let $\left(A^{\prime}, B^{\prime}\right)$ denote the bipartition of $T^{\prime}$ with $A^{\prime} \subseteq A, B^{\prime} \subseteq B$, and define $c_{i j}^{\prime}$ to be the vertex of $\mathcal{H}^{\prime}:=G_{3}\left(T^{\prime}\right)$ with $c_{i j}^{\prime}(a)=i$ for all $a \in A^{\prime}$ and $c_{i j}^{\prime}(b)=j$ for all $b \in B^{\prime}$. By the inductive hypothesis, $\mathcal{H}^{\prime}$ has a Hamilton path between $c_{12}^{\prime}$ and $c_{13}^{\prime}$. Let $V\left(\mathcal{H}^{\prime}\right):=\left\{f_{0}, f_{1}, \ldots, f_{N-1}\right\}$. Since $\mathcal{H}^{\prime}$ has a Hamilton path we may assume that $f_{0} f_{1} \cdots f_{N-1}$ is a Hamilton path in $\mathcal{H}^{\prime}$ between $f_{0}:=c_{12}^{\prime}$ and $f_{N-1}:=c_{13}^{\prime}$.

For $0 \leq i \leq N-1$, let $F_{i}$ be the set of 3-colourings of $\mathcal{H}$ that agree with $f_{i}$ on $V\left(T^{\prime}\right)$. Then $\left\{F_{0}, F_{1}, \ldots, F_{N-1}\right\}$ is a partition of the vertices of $\mathcal{H}$, and $\mathcal{H}\left[F_{i}\right] \cong P_{4} \square P_{4}$, $0 \leq i \leq N-1$. If $f_{i-1}$ and $f_{i}$ differ on the colour of a vertex of $V\left(T^{\prime}\right) \backslash\left\{u^{\prime \prime}, v^{\prime \prime}\right\}$, then $\left|\left[F_{i-1}, F_{i}\right]\right|=16$ and $\mathcal{H}\left[F_{i-1} \cup F_{i}\right] \cong\left(P_{4} \square P_{4}\right) \square K_{2}$. Otherwise, $f_{i-1}$ and $f_{i}$ differ on the colour of $u^{\prime \prime}$ or $v^{\prime \prime}$, implying that $\left|\left[F_{i-1}, F_{i}\right]\right|=8$, and the subgraph of $\mathcal{H}$ induced by the endpoints of the edges of $\left[F_{i-1}, F_{i}\right]$ is $C_{4} \square P_{4}$.

Consider the spanning subgraph $G$ of $\mathcal{H}$ with edge set

$$
\left(\bigcup_{i=0}^{N-1} E\left(\mathcal{H}\left[F_{i}\right]\right)\right) \cup\left(\bigcup_{i=1}^{N-1}\left[F_{i-1}, F_{i}\right]\right)
$$

Note that by Remark A.4, $G$ is a connected $B$-graph. Let $(\mathcal{A}, \mathcal{B})$ be the bipartition of $G$ and assume $c_{12} \in \mathcal{A}$. Since $\mathcal{H}$ is bipartite by Lemma A.2, and $d_{\mathcal{H}}\left(c_{12}, c_{13}\right)$ is odd, $c_{13} \in \mathcal{B}$. As $c_{12} \in F_{0}$ and $c_{13} \in F_{N-1}$, it follows from Corollary A.7 that there is a Hamilton path in $G$ between $c_{12}$ and $c_{13}$.

Base Case 2. Let $T \cong \mathcal{E}_{2 s, 2 t}^{4 k+2}$ or $T \cong \mathcal{E}_{2 s+1,2 t+1}^{4 k}$ with $s, t \geq 1, k \geq 0$, and $T^{\prime} \cong P_{4 k+2}$ be obtained from $T$ by deleting $2 s$ leaves adjacent to $u$ and $2 t$ leaves adjacent to $v$.

Let $\left(A^{\prime}, B^{\prime}\right)$ denote the bipartition of $T^{\prime}$ with $A^{\prime} \subseteq A, B^{\prime} \subseteq B$, and define $c_{i j}^{\prime}$ to be the vertex of $\mathcal{H}^{\prime}:=G_{3}\left(T^{\prime}\right)$ with $c_{i j}^{\prime}(a)=i$ for all $a \in A^{\prime}$ and $c_{i j}^{\prime}(b)=j$ for all $b \in B^{\prime}$. By Case $1, \mathcal{H}^{\prime}$ has a Hamilton path between $c_{12}^{\prime}$ and $c_{13}^{\prime}$. Let $V\left(\mathcal{H}^{\prime}\right):=\left\{f_{0}, f_{1}, \ldots, f_{N-1}\right\}$. Since $\mathcal{H}^{\prime}$ has a Hamilton path we may assume that $f_{0} f_{1} \cdots f_{N-1}$ is a Hamilton path in $\mathcal{H}^{\prime}$ between $f_{0}:=c_{12}^{\prime}$ and $f_{N-1}:=c_{13}^{\prime}$.

For $0 \leq i \leq N-1$, let $F_{i}$ be the set of 3-colourings of $\mathcal{H}:=G_{3}(T)$ that agree with $f_{i}$ on $V\left(T^{\prime}\right)$. Then $\left\{F_{0}, F_{1}, \ldots, F_{N-1}\right\}$ is a partition of the vertices of $\mathcal{H}$, and $\mathcal{H}\left[F_{i}\right] \cong Q_{2 s+2 t}$, $0 \leq i \leq N-1$. If $f_{i-1}$ and $f_{i}$ differ on the colour of a vertex of $V\left(T^{\prime}\right) \backslash\{u, v\}$, then $\left|\left[F_{i-1}, F_{i}\right]\right|=2^{2 s+2 t}$ and $\mathcal{H}\left[F_{i-1} \cup F_{i}\right] \cong Q_{2 s+2 t} \square K_{2} \cong Q_{2 s+2 t+1}$. If $f_{i-1}$ and $f_{i}$ differ on the colour of $u$ then $\left|\left[F_{i-1}, F_{i}\right]\right|=2^{2 t}$, and the subgraph of $\mathcal{H}$ induced by the 
endpoints of the edges of $\left[F_{i-1}, F_{i}\right]$ is $Q_{2 t+1}$. Otherwise $f_{i-1}$ and $f_{i}$ differ on the colour of $v$ implying that $\left|\left[F_{i-1}, F_{i}\right]\right|=2^{2 s}$, and the subgraph of $\mathcal{H}$ induced by the endpoints of the edges of $\left[F_{i-1}, F_{i}\right]$ is $Q_{2 s+1}$.

Consider the spanning subgraph $G$ of $\mathcal{H}$ with edge set

$$
\left(\bigcup_{i=0}^{N-1} E\left(\mathcal{H}\left[F_{i}\right]\right)\right) \cup\left(\bigcup_{i=1}^{N-1}\left[F_{i-1}, F_{i}\right]\right) .
$$

Note that by Remark A.4, $G$ is a connected $B$-graph. Let $(\mathcal{A}, \mathcal{B})$ be the bipartition of $G$ and assume $c_{12} \in \mathcal{A}$. Since $\mathcal{H}$ is bipartite by Lemma A.2, and $d_{\mathcal{H}}\left(c_{12}, c_{13}\right)$ is odd, $c_{13} \in \mathcal{B}$. As $c_{12} \in F_{0}$ and $c_{13} \in F_{N-1}$, it follows from Corollary A.7 that there is a Hamilton path in $G$ between $c_{12}$ and $c_{13}$.

Base Case 3. Let $T \cong \mathcal{E}_{2 s+1,1}^{4 k}$ with $s, k \geq 1$, and let $T^{\prime} \cong P_{4 k-2}$ be obtained from $T$ by deleting the $2 s+2$ leaves and the vertices $u$ and $v$. Define $u^{\prime}, v^{\prime}$ as the leaves of $T^{\prime}$ so that in $T, u^{\prime}$ is adjacent to $u$ and $v^{\prime}$ is adjacent to $v$.

Let $\left(A^{\prime}, B^{\prime}\right)$ denote the bipartition of $T^{\prime}$ with $A^{\prime} \subseteq A, B^{\prime} \subseteq B$, and define $c_{i j}^{\prime}$ to be the vertex of $\mathcal{H}^{\prime}:=G_{3}\left(T^{\prime}\right)$ with $c_{i j}^{\prime}(a)=i$ for all $a \in A^{\prime}$ and $c_{i j}^{\prime}(b)=j$ for all $b \in B^{\prime}$. By Case $1, \mathcal{H}^{\prime}$ has a Hamilton path between $c_{12}^{\prime}$ and $c_{13}^{\prime}$. Let $V\left(\mathcal{H}^{\prime}\right):=\left\{f_{0}, f_{1}, \ldots, f_{N-1}\right\}$. Since $\mathcal{H}^{\prime}$ has a Hamilton path we may assume that $f_{0} f_{1} \cdots f_{N-1}$ is a Hamilton path in $\mathcal{H}^{\prime}$ between $f_{0}:=c_{12}^{\prime}$ and $f_{N-1}:=c_{13}^{\prime}$.

For $0 \leq i \leq N-1$, let $F_{i}$ be the set of 3-colourings of $\mathcal{H}:=G_{3}(T)$ that agree with $f_{i}$ on $V\left(T^{\prime}\right)$. Then $\left\{F_{0}, F_{1}, \ldots, F_{N-1}\right\}$ is a partition of the vertices of $\mathcal{H}$, and $\mathcal{H}\left[F_{i}\right] \supseteq$ $P_{4} \square P_{2^{2 s+2}}, 0 \leq i \leq N-1$. This follows since $G_{L}\left(K_{1,2 s+1}\right)$ has a Hamilton path $P_{2^{2 s+2}}$ where $L$ is an assignment of lists in which vertices of degree one have lists $\{1,2,3\}$ and the remaining vertex has list $\{1,2\}$. If $f_{i-1}$ and $f_{i}$ differ on the colour of a vertex of $V\left(T^{\prime}\right) \backslash$ $\left\{u^{\prime}, v^{\prime}\right\}$, then $\left|\left[F_{i-1}, F_{i}\right]\right|=4 \cdot 2^{2 s+1}$ and $\mathcal{H}\left[F_{i-1} \cup F_{i}\right] \supseteq\left(P_{4} \square P_{2^{2 s+1}}\right) \square K_{2}$. If $f_{i-1}$ and $f_{i}$ differ on the colour of $v^{\prime}$ then $\left|\left[F_{i-1}, F_{i}\right]\right|=2 \cdot 2^{2 s+1}$, and the subgraph of $\mathcal{H}$ induced by the endpoints of the edges of $\left[F_{i-1}, F_{i}\right]$ contains $\left(P_{2} \square P_{2^{2 s+1}}\right) \square K_{2}$. Otherwise $f_{i-1}$ and $f_{i}$ differ on the colour of $u^{\prime}$ implying that $\left|\left[F_{i-1}, F_{i}\right]\right|=4 \cdot 2^{2 s}$, and the subgraph of $\mathcal{H}$ induced by the endpoints of the edges of $\left[F_{i-1}, F_{i}\right]$ contains $\left(P_{4} \square P_{2^{2 s}}\right) \square K_{2}$.

Consider the spanning subgraph $G$ of $\mathcal{H}$ with edge set

$$
\left(\bigcup_{i=0}^{N-1} E\left(\mathcal{H}\left[F_{i}\right]\right)\right) \cup\left(\bigcup_{i=1}^{N-1}\left[F_{i-1}, F_{i}\right]\right) \text {. }
$$

Note that by Remark A.4, $G$ is a connected $B$-graph . Let $(\mathcal{A}, \mathcal{B})$ be the bipartition of $G$ and assume $c_{12} \in \mathcal{A}$. Since $\mathcal{H}$ is bipartite by Lemma A.2, and $d_{\mathcal{H}}\left(c_{12}, c_{13}\right)$ is odd, $c_{13} \in \mathcal{B}$. As $c_{12} \in F_{0}$ and $c_{13} \in F_{N-1}$, it follows from Corollary A.7 that there is a Hamilton path in $G$ between $c_{12}$ and $c_{13}$.

Induction Step. Now suppose $T$ is a tree with bipartition $(A, B)$, where $|A|:=\ell>1$ and $|B|:=r>1$ are both odd and $T$ is not isomorphic to any of the graphs in Base Cases 1 to 3. If every pair of leaves $x, y \in A$ or $x, y \in B$ satisfy $d_{T}(x, y) \leq 2$, then $T \cong \mathcal{E}_{s, t}^{k}$; since $\ell, r>1$ are both odd, $T$ is isomorphic to one of the graphs in Base Cases 1 to 3. Thus, there are leaves $x, y \in A$ (or $x, y \in B$ ) with $d_{T}(x, y) \geq 3$.

Case 1. If $T-\{x, y\}$ is a star, then $T$ is the graph obtained from $K_{1,2 s+1}, s \geq 1$, by subdividing two of its edges. Let $u \in V(T)$ be the vertex of degree $2 s+1$ and $v \in V(T)$ a leaf adjacent to $u$. Define $T^{\prime}:=T[\{u, v\}]$ and observe $T^{\prime} \cong K_{2}$. 
Let $\mathcal{H}^{\prime}:=G_{3}\left(T^{\prime}\right)$ have Hamilton path $f_{0} f_{1} f_{2} f_{3} f_{4} f_{5}:=c_{12}^{\prime} c_{32}^{\prime} c_{31}^{\prime} c_{21}^{\prime} c_{23}^{\prime} c_{13}^{\prime}$, where $c_{i j}^{\prime}(u)=i$ and $c_{i j}^{\prime}(v)=j$. For $0 \leq i \leq 5$, let $F_{i}$ be the set of 3 -colourings of $\mathcal{H}:=G_{3}(T)$ that agree with $f_{i}$ on $V\left(T^{\prime}\right)$. Then $\left\{F_{0}, F_{1}, \ldots, F_{5}\right\}$ is a partition of the vertices of $\mathcal{H}$, and $\mathcal{H}\left[F_{i}\right] \cong P_{4} \square P_{4} \square Q_{2 s-2}, 0 \leq i \leq 5$. If $f_{i-1}$ and $f_{i}$ differ on the colour of vertex $v$, then $\left|\left[F_{i-1}, F_{i}\right]\right|=4 \cdot 4 \cdot 2^{2 s-2}$ and $\mathcal{H}\left[F_{i-1} \cup F_{i}\right]=\left(P_{4} \square P_{4} \square Q_{2 s-2}\right) \square K_{2}$. If $f_{i-1}$ and $f_{i}$ differ on the colour of $u$ then $\left|\left[F_{i-1}, F_{i}\right]\right|=4$, and the subgraph of $\mathcal{H}$ induced by the endpoints of the edges of $\left[F_{i-1}, F_{i}\right]$ is $C_{4}$.

Consider the spanning subgraph $G$ of $\mathcal{H}$ with edge set

$$
\left(\bigcup_{i=0}^{5} E\left(\mathcal{H}\left[F_{i}\right]\right)\right) \cup\left(\bigcup_{i=1}^{5}\left[F_{i-1}, F_{i}\right]\right) .
$$

Note that by Remark A.4, the graph $P_{4} \square P_{4} \square Q_{2 s-2}$ is Hamilton laceable since $P_{4} \square$ $P_{4} \square P_{2^{2 s-2}}$ is a Hamilton laceable spanning subgraph. Thus, $G$ is a connected $B$-graph. Let $(\mathcal{A}, \mathcal{B})$ be the bipartition of $G$ and assume $c_{12} \in \mathcal{A}$. Since $\mathcal{H}$ is bipartite by Lemma A.2, and $d_{\mathcal{H}}\left(c_{12}, c_{13}\right)$ is odd, $c_{13} \in \mathcal{B}$. As $c_{12} \in F_{0}$ and $c_{13} \in F_{5}$, it follows from Corollary A.7 that there is a Hamilton path in $G$ between $c_{12}$ and $c_{13}$.

Case 2. Suppose $N_{T}(x):=\left\{x^{\prime}\right\}$ and $N_{T}(y):=\left\{y^{\prime}\right\}$, and that $T^{\prime}:=T-\{x, y\}$ is not a star. Let $\left(A^{\prime}, B^{\prime}\right)$ denote the bipartition of $T^{\prime}$ with $A^{\prime} \subseteq A, B^{\prime} \subseteq B$, and define $c_{i j}^{\prime}$ to be the vertex of $\mathcal{H}^{\prime}:=G_{3}\left(T^{\prime}\right)$ with $c_{i j}^{\prime}(a)=i$ for all $a \in A^{\prime}$ and $c_{i j}^{\prime}(b)=j$ for all $b \in B^{\prime}$.

By the inductive hypothesis, $\mathcal{H}^{\prime}$ has a Hamilton path between $c_{12}^{\prime}$ and $c_{13}^{\prime}$. Let $V\left(\mathcal{H}^{\prime}\right):=$ $\left\{f_{0}, f_{1}, \ldots, f_{N-1}\right\}$. Since $\mathcal{H}^{\prime}$ has a Hamilton path we may assume that $f_{0} f_{1} \cdots f_{N-1}$ is a Hamilton path in $\mathcal{H}^{\prime}$ between $f_{0}:=c_{12}^{\prime}$ and $f_{N-1}:=c_{13}^{\prime}$.

For $0 \leq i \leq N-1$, let $F_{i}$ be the set of 3-colourings of $\mathcal{H}:=G_{3}(T)$ that agree with $f_{i}$ on $V\left(T^{\prime}\right)$. Then $\left\{F_{0}, F_{1}, \ldots, F_{N-1}\right\}$ is a partition of the vertices of $\mathcal{H}$, and $\mathcal{H}\left[F_{i}\right] \cong C_{4}$, $0 \leq i \leq N-1$. If $f_{i-1}$ and $f_{i}$ differ on the colour of a vertex of $V\left(T^{\prime}\right) \backslash\left\{x^{\prime}, y^{\prime}\right\}$, then $\left|\left[F_{i-1}, F_{i}\right]\right|=4$ and $\mathcal{H}\left[F_{i-1} \cup F_{i}\right] \cong Q_{3}$. Otherwise, $f_{i-1}$ and $f_{i}$ differ on the colour of $x^{\prime}$ or $y^{\prime}$, implying that $\left|\left[F_{i-1}, F_{i}\right]\right|=2$, the subgraph of $\mathcal{H}$ induced by the endpoints of the edges of $\left[F_{i-1}, F_{i}\right]$ is a 4 -cycle, and $\mathcal{H}\left[F_{i-1} \cup F_{i}\right] \cong P_{4} \square K_{2}$.

Consider the spanning subgraph $G$ of $\mathcal{H}$ with edge set

$$
\left(\bigcup_{i=0}^{N-1} E\left(\mathcal{H}\left[F_{i}\right]\right)\right) \cup\left(\bigcup_{i=1}^{N-1}\left[F_{i-1}, F_{i}\right]\right)
$$

Note that $G$ is a connected $B$-graph. Let $(\mathcal{A}, \mathcal{B})$ be the bipartition of $G$ and assume $c_{12} \in$ $\mathcal{A}$. Since $\mathcal{H}$ is bipartite by Lemma A.2 and $d_{\mathcal{H}}\left(c_{12}, c_{13}\right)$ is odd, $c_{13} \in \mathcal{B}$. As $c_{12} \in F_{0}$ and $c_{13} \in F_{N-1}$, it follows from Corollary A.7 that there is a Hamilton path in $G$ between $c_{12}$ and $c_{13}$. 